PHYSICAL ACTIVITY AND SPINAL CORD INJURY REHABILITATION 


\title{
INCORPORATING PHYSICAL ACTIVITY INTO THE REHABILITATION PROCESS AFTER SPINAL CORD INJURY
}

\author{
By: CHELSEA A. PELLETIER, MSc, BKINH
}

A Thesis Submitted to the School of Graduate Studies in Partial Fulfillment of the Requirements for the Degree Doctor of Philosophy 
PhD Thesis - Chelsea A. Pelletier - McMaster University - Kinesiology

McMaster University DOCTOR OF PHILOSOPHY (2013) Hamilton, Ontario (Kinesiology)

TITLE: Incorporating Physical Activity into the Rehabilitation Process after Spinal Cord Injury

AUTHOR: Chelsea A. Pelletier, MSc (McMaster University), BKINH (Acadia University)

SUPERVISOR: Dr. Audrey L. Hicks

NUMBER OF PAGES: xi, 137 


\begin{abstract}
It is well established that physical activity can improve aspects of physical fitness in individuals with spinal cord injury (SCI). Despite reports of declining health and fitness post-discharge from rehabilitation, there is a limited amount of research exploring exercise status or interventions during this period. The purpose of this dissertation was to investigate the integration of structured exercise into the rehabilitation process following SCI, and to optimize the exercise prescription in the community setting. Findings from the first study indicated that exercise is well tolerated among individuals with sub-acute SCI; performance of a peak exercise test on an arm ergometer was feasible for all injury types. At this stage post-injury, interventions should be mindful of the greater risk of orthostatic intolerance in individuals with complete tetraplegia and focus on building task specific self-efficacy. The second study involved a direct referral and physical activity counselling intervention post-discharge. Adherence rates were excellent among those participants who received the intervention suggesting that this model of care can facilitate adherence to community exercise after discharge.

The final two studies took place in the community. Several modes of adapted exercise were compared and findings indicated that while there were no differences in measures of physiological intensity or enjoyment between the different modes, arm-only exercise was perceived as safer than passive hybrid (arm and leg) exercise. Further, the validity of using ratings of perceived exertion (RPE) to attain prescribed exercise intensity was established. The efficacy of the physical activity guidelines for improving fitness in adults with SCI were evaluated in a community-based randomized controlled trial and the results revealed that the guidelines were effective in improving both aerobic capacity and muscle strength. Taken together, this series of studies describes a model of care that links rehabilitation with community exercise and suggests options for sustained engagement.
\end{abstract}




\section{Acknowledgments}

I would first like to thank and acknowledge the assistance of my supervisor, Audrey Hicks. Six years has gone by so fast and I have sincerely appreciated your endless support and all of the time you have spent editing my nonsense and giving me pep talks when I needed them.

I would also like to acknowledge the advice and support of my committee: Dave Ditor, Kathleen Martin Ginis, and Neil McCartney, and include Amy Latimer-Cheung for her collaboration and patience.

The projects described in this dissertation have been the result of numerous collaborations that have been essential to their success. The staff at Hamilton Health Sciences Regional Rehabilitation Hospital were vital for the completion of the first two projects. In particular, I must acknowledge Dr. Graham Jones, Michelle Read, and Dianne Schaible who gave so much of their time to help me with testing and recruitment. These projects would also not have been possible without many research assistants and students at various study sites, namely: Renne Fougere, Teri Lynch, and Jessica Tat. Finally, the members of the Vascular Dynamics Lab who have been (and continue to be) great friends and collaborators, especially Julia Totosy de Zepetnek and Jason Au, and my own lab members Alyssa Fenuta and Lara Pilutti.

I would like to express genuine appreciation to all the staff, volunteers, and members of MacWheelers. There have been too many faces over the years to name, but none of this work would have been possible without your continued enthusiasm and commitment. Particularly to the fearless leaders, and my good friends, Susie Ward and Jenn Hohol who have always been so generous with their time and display compassion and kindness that I have witnessed with great admiration. Finally, thank you to each of my participants for their patience, dedication, and friendship.

Of course, as no research ever could be, these projects would not have been possible without the support from our funding sources, the Ontario Neurotrauma Foundation and the Risk Hansen Institute. 


\title{
Table of Contents
}

\author{
Abstract \\ Acknowledgements \\ Table of Contents \\ List of Figures \\ List of Tables \\ List of Abbreviations \\ Contribution to Papers with Multiple Authors
}

Chapter 1. Introduction and Literature Review

1. Spinal Cord Injury

1.1 Prevalence and Epidemiology

1.2 Changes in Health and Disease Risk after Spinal Cord Injury

2. Spinal Cord Injury Rehabilitation

iv V

Exercise Perceptions at Discharge from Inpatient Spinal Cord Injury Rehabilitation

Chapter 3. Direct Referral and Physical Activity Counselling upon Discharge from Spinal Cord Injury Rehabilitation

Chapter 4. The Optimal Modes of Exercise for Individuals with Spinal Cord Injury: Consumer Preference and Metabolic Demand 
PhD Thesis - Chelsea A. Pelletier - McMaster University - Kinesiology

Chapter 5. Implementation of the Physical Activity

Guidelines for Adults with Spinal Cord Injury: Effects on

Aerobic Capacity and Muscle Strength

Chapter 6. Discussion and Conclusions

\section{Appendices.}

A. Chapter 2

A.1 Exercise Self-efficacy Questionnaire

B. Chapter 3

B.1 Exercise Beliefs Questionnaire

127

B.2 Exercise Referral Form

131

C. Chapter 4

C.1 Consumer Preference Questionnaire

132

D. Chapter 5

D.1 Satisfaction with the Guidelines Questionnaire

137 


\section{List of Figures}

\section{Chapter 2}

Figure 1. Mean SBP and DBP changes during a 30-minute sit-up test in participants with TP, HP, and LP

\section{Chapter 3}

Figure 1. Adherence rate ( $\%$ of prescribed sessions) for each participant during a 16-week follow-up period

\section{Chapter 4}

Figure 1. Heart rate during 20-minutes of submaximal steady state exercise in participants with TP and PP

Figure 2. Oxygen consumption during 20-minutes of submaximal steady state exercise in participants with TP and PP

\section{Chapter 5}

Figure 1. Muscle endurance following 16-weeks of exercise training 


\section{List of Tables}

\section{Chapter 2}

Table 1. Participant characteristics 34

Table 2. Results of the peak exercise test

Table 3. Prevalence of $\mathrm{OH}$ based on level and severity of injury 35

Table 4. Blood pressure values before and after postural challenge 36

Table 5. Mean composite scores for outcome value, outcome expectation, 36

scheduling, and task self-efficacy

\section{Chapter 3}

Table 1. Participant characteristics

Table 2. Prevalence of barriers to physical activity participation 58

Table 3. Mean composite scores for outcome value, outcome expectations, 59

scheduling and task self-efficacy

\section{Chapter 4}

Table 1. Participant characteristics $\quad 81$

Table 2. Results of the peak exercise test $\quad 82$

Table 3. Physiological variables during steady state submaximal exercise $\quad 83$

Table 4. Ratings of perceived exertion (10-point scale) during 20-minutes of 84 steady state submaximal exercise

Table 5. Results of consumer preference questionnaire $\quad 85$

\section{Chapter 5}

Table 1. Participant characteristics 108

Table 2. Peak aerobic capacity pre and post 16-weeks of exercise training 109

Table 3. Aerobic endurance pre and post 16-weeks of exercise training 110

Table 4. Muscle strength pre and post 16-weeks of exercise training 111 


\section{List of Abbreviations}

$\begin{array}{ll}\text { 1RM } & \text { One Repetition Maximum } \\ \text { ACE } & \text { Arm Cycle Ergometry } \\ \text { AG } & \text { Arm Glider } \\ \text { AIS } & \text { American Spinal Injury Association Impairment Scale } \\ \text { ALCE } & \text { Arm + Leg Cycle Ergometer } \\ \text { ALRS } & \text { Arm + Leg Recumbent Stepper } \\ \text { ANOVA } & \text { Analysis of Variance } \\ \text { BMI } & \text { Body Mass Index } \\ \text { CON } & \text { Control } \\ \text { CSA } & \text { Cross-Sectional Area } \\ \text { DBP } & \text { Diastolic Blood Pressure } \\ \text { ECG } & \text { Electrocardiogram } \\ \text { FES } & \text { Functional Electrical Stimulation } \\ \text { HP } & \text { High Paraplegia } \\ \text { HR } & \text { Heart Rate } \\ \text { LP } & \text { Low Paraplegia } \\ \text { LTPA } & \text { Leisure Time Physical Activity } \\ \text { MET } & \text { Metabolic Equivalent } \\ \text { NMES } & \text { Neuromuscular Electrical Stimulation } \\ \text { OH } & \text { Orthostatic Hypotension } \\ \text { PAG } & \text { Physical Activity Guidelines } \\ \text { PO } & \text { Power Output } \\ \text { PP } & \text { Paraplegia } \\ \text { RER } & \text { Respiratory Exchange Ratio } \\ \text { RPE } & \text { Rating of Perceived Exertion } \\ \text { SBP } & \text { Systolic Blood Pressure } \\ \text { SCI } & \text { Spinal Cord Injury } \\ \text { SE } & \text { Self-Efficacy } \\ \text { TP } & \text { Tetraplegia } \\ \text { VE } & \text { Minute Ventilation } \\ \text { VO } & \text { Oxygen Consumption } \\ \text { WCE } & \text { Wheelchair Ergometry } \\ \text { WP } & \text { Wall Pulleys } \\ \text { WS } & \text { Weight Stack } \\ & \end{array}$




\section{Contribution to Papers with Multiple Authors}

Please be advised that several of the manuscripts within this dissertation (Chapters 2-5) have been prepared for journal publication with multiple authors.

\section{Chapter 2.}

Pelletier CA, Jones G, Latimer-Cheung AE, Warburton DE, Hicks AL. Aerobic capacity, orthostatic tolerance, and exercise perceptions at discharge from inpatient spinal cord injury rehabilitation. Arch Phys Med Rehabil 2013, doi:10.1016/j.apmr.2013.05.011

\section{Contribution}

CAP, AELC, DEW, ALH were involved in study conception and design. Testing protocol and consistency between study sites was overseen by CAP and ALH. Testing was completed by CAP and GJ at the Hamilton study site, and DEW and research assistant Renee Fougere at the Vancouver site. Statistical analysis and manuscript preparation was completed by CAP, with editing contributions from GJ, AELC, DEW and ALH.

\section{Chapter 3.}

Pelletier CA, Latimer-Cheung AE, Warburton DE, Hicks AL. Direct referral and physical activity counselling upon discharge from spinal cord injury rehabilitation.

\section{Contribution}

Study protocol and materials were developed by CAP, AELC, and ALH. The counselling intervention was completed by CAP at the Hamilton, Ontario study site and research assistant Renee Fougere (supervised by DEW) at the Vancouver, British Columbia site. Statistical analysis and manuscript preparation was completed by CAP and edited by ALH.

\section{Chapter 4.}

Pelletier CA, Ditor DS, Latimer-Cheung AE, Warburton DE, Hicks AL. The optimal modes of exercise for individuals with spinal cord injury: consumer preference and metabolic demand.

\section{Contribution}

CAP, DSD, AELC, DEW, ALH were involved in study conception and design. Testing was overseen or completed at the various study sites by CAP (Hamilton, Ontario), DDS (St. Catherine's, Ontario), AELC (Kingston, Ontario), and DEW (Vancouver, British Columbia). Statistical analysis and manuscript preparation was completed by CAP and edited by ALH.

\section{Chapter 5.}

Pelletier CA, Totosy de Zepetnek J, MacDonald M, Hicks AL. Implementation of the physical activity guidelines for adults with spinal cord injury: effects on aerobic capacity and muscle strength. 
PhD Thesis - Chelsea A. Pelletier - McMaster University - Kinesiology

\section{Contribution}

CAP, JTdZ, MM, ALH were involved in study conception and design. Exercise testing and training was completed by CAP and JTdZ. Statistical analysis and manuscript preparation was completed by CAP and edited by ALH. 
PhD Thesis - Chelsea A. Pelletier - McMaster University - Kinesiology

Chapter 1

Introduction and Literature Review 


\section{Spinal Cord Injury}

\subsection{Prevalence and Epidemiology}

The prevalence of spinal cord injury (SCI) in Canada is estimated at 1289 persons per million. There are approximately 43974 individuals currently living with an SCI, and this number is nearly doubled to 85556 if non-traumatic causes are included. ${ }^{1,2}$ The highest prevalence rates occur between ages $20-40$, with a mean age at injury of 42.2 years. ${ }^{1,3}$ However, owing to an increasingly aging population, a greater percentage of new injuries is occurring among individuals over the age of 60 , and the age adjusted incidence rates are actually highest in this demographic. ${ }^{3,4}$ This increasing age at injury has potential implications for both the acute care and rehabilitation of people with SCI as it increases complication rates, the potential of existing medical conditions, length of hospital stay, and decreases long-term functional ability and recovery. ${ }^{3,4}$ In Canada, the most common cause of SCIs are motor vehicle accidents (35\%), falls (31\%), other vehicle accidents $(12 \%)$, and sports/recreation accidents $(9 \%){ }^{3}$

Classifying SCIs involves the identification of which spinal segment (cervical, thoracic, lumbar, or sacral) is damaged and to what extent motor and sensory function is preserved. There are two general categories of SCI: tetraplegia, which encompasses all cervical level lesions (C1-C8), and paraplegia, which includes all lesions occurring in the thoracic, lumbar, or sacral regions. Often, paraplegia is further divided into two categories: high thoracic/paraplegia (T1-T6) and low thoracic/paraplegia (T7 and below). In Canada, it is estimated that there are currently 24742 people living with tetraplegia and 19232 people 
living with paraplegia from traumatic causes. ${ }^{1,2}$ These statistics are reflective of epidemiological reports indicating the $56.7 \%$ of new injuries occur in the cervical segment and $43.3 \%$ at or below the thoracic segment of the spine. ${ }^{1,2,4}$

In addition to level of injury, SCIs are also classified according to the severity or completeness of injury. The American Spinal Injury Association impairment scale (AIS) is the standard classification scheme used to describe completeness of injury and uses a scoring system from A-D. AIS A refers to a complete injury, with no motor or sensory function preserved in the sacral segments S4-S5. ${ }^{5}$ AIS B is an incomplete injury, with sensory but not motor function preserved below the neurological level of injury, including sacral segments S4-S5. AIS C is also an incomplete injury, when both motor and sensory functions are preserved, but with more than half of the key muscles below the level of the lesion having a muscle grade less than $3 .^{5}$ AIS D refers to incomplete injuries when motor function is preserved below the level of the lesion but at least half of the key muscle below the neurological level have a muscle grade of 3 or more. ${ }^{5}$

Finally, SCIs are divided based on time post-injury. An acute SCI is typically defined as the time period spanning up until one-year post injury and is considered to be the phase when the most significant and rapid changes to physiological function occur. Sub-acute $\mathrm{SCI}$ is considered to be the time during which rehabilitation is occurring. After one-year post injury, injuries are classified as chronic and physiological changes considered stable. 


\subsection{Changes in Health and Disease Risk after Spinal Cord Injury}

Physiological changes after SCI are widespread and extend to all major bodily systems through dysfunctions in efferent and afferent neurological pathways. The consequential disruptions in motor, sensory, and autonomic functions have a significant impact on physical functioning, quality of life, and longevity. The profound physical deconditioning secondary to SCI is due to a combination of factors including paralysis and loss of voluntary motor control, but also an increased tendency for sedentary behaviour. This deconditioning creates a cycle of decreasing physical capacity leading to decreased activity, and so on. These factors combined with a negative shift in body composition lead to an increased cardiometabolic disease risk.

Cardiovascular disease is the leading cause of mortality in persons with an injury more than 30 years in duration. ${ }^{6}$ The risk of cardiovascular disease after SCI is $16 \%$ greater among individuals with tetraplegia, and the presence of a complete injury results in a $44 \%$ increased risk. ${ }^{7}$ The prevalence of metabolic syndrome is $23 \%$ in individuals beyond the first year post injury, a percentage which is nearly double that reported in the able-bodied population. ${ }^{8}$

Cross-sectional imaging studies have revealed that mean percent fat mass is $23-35 \%$ in men with chronic SCI, representing an 8-18\% increase compared to age-, height-, and weight-matched controls. ${ }^{9-12}$ While body mass index $\left(\mathrm{BMI} ; \mathrm{kg} / \mathrm{m}^{2}\right)$ is traditionally used to 
indicate weight status and disease risk in the able-bodied population, it has been suggested that traditional classifications of overweight $\left(27 \mathrm{~kg} / \mathrm{m}^{2}\right)$ and obesity $\left(30 \mathrm{~kg} / \mathrm{m}^{2}\right)$ be lowered for persons with SCI to reflect the decreased lean tissue mass and increased fat mass that are indistinguishable with traditional measures of body weight. ${ }^{10-13}$ Based on percentage of fat mass and biomarkers of inflammation, a BMI cut-off of $30 \mathrm{~kg} / \mathrm{m}^{2}$ fails to recognize $73.9 \%$ of obese individuals with SCI, while a more conservative cut-off of $22 \mathrm{~kg} / \mathrm{m}^{2}$ more accurately identifies those at increased risk for cardiovascular disease. ${ }^{13}$ Longitudinal studies have revealed that BMI increases significantly in the year immediately following discharge from inpatient SCI rehabilitation and up to 5 years later by $1.8 \mathrm{~kg} / \mathrm{m}^{2}$, with a rise from $56 \%$ to $75 \%$ of the population being identified as overweight or obese. ${ }^{14,15}$ This change occurs simultaneously with muscle atrophy of 27$56 \%,{ }^{16,17}$ and a decrease in physical activity by $33 \% .^{18}$

\section{Spinal Cord Injury Rehabilitation}

After SCI, individuals typically go through several phases of rehabilitation including acute care, inpatient rehabilitation, and outpatient rehabilitation services. In Canada, the discharge incidence from inpatient rehabilitation in 2010 was 1389 (41 per million) for traumatic and 2286 for non-traumatic SCI. ${ }^{1}$ Currently, the average length of stay in inpatient rehabilitation is 103 days (range: 41 - 336) with extensions typically resulting from increased complexity of injuries and secondary complications. ${ }^{19}$ At discharge, $42 \%$ of individuals return home (45\% traumatic, $38 \%$ non-traumatic), while only $15 \%$ report 
receiving outpatient rehabilitation services. ${ }^{19}$ It is estimated that the average health care cost of inpatient rehabilitation for traumatic SCI is $\$ 119945$ per person. ${ }^{20}$

Current trends in rehabilitation practice are to decrease length of inpatient stay and increase the number of services received post-discharge. ${ }^{21,22}$ While this increased reliance on outpatient programs serves primarily to decrease cost, it has a potential long-term influence by hindering reintegration into the community. Services to enhance community participation are limited during the transitional period post-discharge, at a time when new obstacles to community participation appear. ${ }^{23}$ Reintegration and perceived control are positively influenced by educational programs, although limited long-term communitybased outpatient services diminish the opportunity for sustained effects. ${ }^{24}$ Recently, Rimmer $^{25}$ described a projected health trajectory for individuals as they complete rehabilitation depicting a short-term gain in health and function during rehabilitation, followed by a plateau around the time of discharge. At twelve weeks post-discharge, an inflection point occurs which can be characterised by a decrease or increase in function depending on physical activity participation. ${ }^{25}$

\section{Exercise Capacity after Spinal Cord Injury}

Decreases in exercise capacity after SCI are well documented and include a decline in both cardiorespiratory aerobic fitness and muscle strength. Exercise capacity has many important implications as illustrated by demonstrated relationships with functional 
ability, ${ }^{26,27}$ wheelchair skill performance, ${ }^{28}$ strain during activities of daily living, ${ }^{29}$ and return to work. ${ }^{30}$

\subsection{Physical Capacity}

Physical capacity is typically defined in terms of peak oxygen uptake $\left(\mathrm{VO}_{2 \text { peak }}\right)$ and peak power output $\left(\mathrm{PO}_{\text {peak }}\right)$ achieved during a maximal exercise test. In the SCI population, the reliance on upper body exercise creates challenges for the accurate assessment of aerobic fitness as the smaller active muscle mass makes it difficult to achieve a true assessment of peak cardiovascular fitness. Nonetheless, graded exercise tests can still be performed using wheelchair ergometry (WCE) or arm cycle ergometry (ACE).

In a review of physical capacity in people with chronic SCI, Haisma et al. ${ }^{31}$ calculated a weighted mean of $2.10 \mathrm{~L} / \mathrm{min}$ (range: 1.10-2.51 L/min) during WCE and $1.51 \mathrm{~L} / \mathrm{min}$ (range: $1.03-2.34 \mathrm{~L} / \mathrm{min}$ ) during ACE in participants with paraplegia. Among participants with tetraplegia, scores were $0.89 \mathrm{~L} / \mathrm{min}$ (range: $0.76-1.03 \mathrm{~L} / \mathrm{min}$ ) and $0.87 \mathrm{~L} / \mathrm{min}$ (range: 0.78-0.95 L/min) for WCE and ACE, respectively. In another review, Janssen $e^{a l} .^{32}$ developed normative values for both $\mathrm{VO}_{2 \text { peak }}$ and $\mathrm{PO}_{\text {peak }}$. The most important factor in predicting physical capacity was lesion level (37-47\% variance explained), with each level lower representing an increase of $0.06 \mathrm{~L} / \mathrm{min}$. Individuals with incomplete lesions also had, on average, a $0.21 \mathrm{~L} /$ min higher $\mathrm{VO}_{2 \text { peak }}$ compared to individuals with complete lesions. Shoulder flexion strength has been shown to significantly correlate with $\mathrm{VO}_{2 \text { peak }}$ 
and $\mathrm{PO}_{\text {peak }}$, with overall shoulder strength accounting for $68.4 \%$ of the variance in performance time..$^{33}$

While there have been few studies examining the physical capacity among adults with acute SCI, longitudinal investigations have revealed improvements both during inpatient rehabilitation and in 1 year post-discharge, especially among individuals with tetraplegia. ${ }^{34} \mathrm{VO}_{2 \text { peak }}$ during rehabilitation also appears to be the best predictor of future $\mathrm{VO}_{2 \text { peak }}{ }^{35}$ and is negatively associated with complications post-discharge. ${ }^{36}$

\subsection{Muscle Strength and Morphology}

Alterations in skeletal muscle structure and function are some of the earliest and most significant changes that occur following SCI. Most prominent is a detrimental and substantial loss of muscle mass. Changes are also observed in muscle fibre composition toward fast fatiguable fibre type (Type IIa or IIx) and changes in muscle contractile properties, both of which contribute to a decrease in fatigue resistance, physical capacity, and functional independence..$^{37,38}$

Muscle atrophy after SCI is represented by a decrease in cross-sectional area (CSA) resulting from both a loss in the overall number of muscle fibers and shrinking in size of the fibres that remain. After complete SCI, muscle CSA has been reported as only 45$80 \%$ of age- and sex- matched able-bodied controls, ${ }^{16,39}$ and is documented as early as 6 
weeks post-injury. ${ }^{16,17}$ After incomplete SCI, the decrease is $24-31 \%$ and is greatest in the upper leg muscles. ${ }^{40}$ The rapid decrease in muscle mass has been shown to stabilize approximately 11 months post-injury. ${ }^{41,42}$ The loss of muscle CSA is accompanied by a $126 \%$ increase in absolute and a three-fold increase in relative intramuscular fat, ${ }^{43,44}$ placing individuals at increased risk for glucose intolerance and type 2 diabetes. ${ }^{45}$ Both of these changes have been shown to vary based on level of injury, and spasticity appears to provide a protective effect. ${ }^{44}$

There are several theories proposed to explain the increased fatiguabily of skeletal muscle after SCI. One of the most obvious relates to the change in fibre type composition postinjury: an increase in the percentage of type II muscle fibres and a decrease in the percentage of type I fibres. This transition occurs mostly within the first year and may not stabilize until 5.8 years post-injury. ${ }^{46}$ Faster rates of muscle contraction and relaxation have been noted in the quadriceps femoris, ${ }^{47}$ although this is not a consistent finding in tibialis anterior or soleus muscle..$^{48-50}$

Few studies have evaluated changes in muscle strength following SCI. Using electrical stimulation, studies have demonstrated a decrease in muscle torque produced by the quadriceps femoris ${ }^{51}$ and vastus lateralis, ${ }^{47}$ while there appears to be no change in the tibialis anterior muscle, ${ }^{49,50}$ and inconsistent findings in the soleus. ${ }^{49,50,52}$ A limited number of studies have examined voluntary muscle torque after SCI, but decreases have been observed in knee extensor $^{52}$ and triceps strength in people with incomplete injuries. ${ }^{53}$ 


\subsection{Autonomic Function}

In addition to the loss of voluntary muscle control, another significant change after SCI is the presence of autonomic dysfunction. Depending on the level of injury, this dysfunction creates many daily challenges in areas of blood pressure regulation, bladder and bowel management, and respiration. Because preganglionic neurons from the parasympathetic nervous system originate in the brainstem and sacral segments $\mathrm{S} 2-\mathrm{S} 4$, this branch of the autonomic nervous system is largely unaffected by SCI. Sympathetic preganglionic neurons reside in thoracic and upper lumbar sections T1-L2 and can be impaired following SCI. In particular, the heart has dual innervation from the parasympathetic (vagus nerve) and sympathetic nervous system (T1-T5). Any SCI originating at or above these spinal segments contributes to changes in heart rate and blood pressure regulation at rest, a blunted response during exercise, and an impairment in heart rate recovery. ${ }^{54,55}$

While the mean resting blood pressure and heart rate is lower among individuals with lesions above T6, these individuals are prone to life-threatening episodes of extreme hypertension (up to $300 \mathrm{mmHg}$ systolic blood pressure), known as autonomic dysreflexia. ${ }^{56}$ This sudden increase in blood pressure is typically provoked by noxious stimuli below the level of the lesion, such as bowel and bladder distension, spasms, or bladder catheterization. On the opposite end of the spectrum, individuals with complete injuries above T6 are also prone to orthostatic hypotension, which is defined as a drop of $20 \mathrm{mmHg}$ systolic and/or $10 \mathrm{mmHg}$ diastolic blood pressure following a change to an 
upright posture. ${ }^{57-59}$ This decrease in blood pressure is often associated with symptoms of lightheadednes, dizziness, and blurred vision and is most common in the acute phase of injury. ${ }^{57,58,60}$ In the first month post-injury, $74 \%$ of persons with cervical SCI experience orthostatic hypotension, while prevalence has been reported at $23 \%$ among those with longer standing injuries..$^{60,61}$

\section{Exercise Interventions after Spinal Cord Injury}

Leisure time physical activity (LTPA) is defined as any activity (mild, moderate, or heavy intensity) that individuals choose to do in their free time. Despite reports of $80-90 \%$ of individuals expressing interest in exercise and perceiving benefits of regular participation, ${ }^{62,63}$ LTPA rates of any intensity are exceedingly low in the SCI population, with a mean of $27.14 \pm 49.36$ minutes per day, and up to $50 \%$ of individuals report no participation. ${ }^{62,64}$ Among non-exercisers, $86 \%$ report wanting to begin an exercise program, yet not having the knowledge or resources to do so. ${ }^{62,65}$ Among people who selfreport participating in LTPA, the greatest time is spent at a moderate intensity, with the smallest at a heavy intensity. ${ }^{66}$ Trends show that a longer time post-injury is associated with a decrease in participation in LTPA. ${ }^{64}$ A sharp decline (33\%) occurs in the months immediately following discharge from inpatient rehabilitation. ${ }^{18}$

There are numerous barriers to exercise participation among individuals with SCI, although internal/intrinsic barriers (i.e., lack of time and energy) and resource barriers are 
reportedly the most strongly associated with exercise participation. ${ }^{62,67}$ Exercise perceptions (i.e., not liking exercise, not valuing exercise, not feeling it could help you) reduce the odds of being an exerciser more than resource or structural barriers (i.e., lack of in-home equipment, inability to pay for transportation or program costs). ${ }^{62}$ Barriers to exercise are reported as most important in the three months following discharge from rehabilitation and include emotional distress, problems with self-care, and mental health problems. $^{63}$

\subsection{Exercise Interventions to Improve Health and Disease Risk}

While the link between exercise participation and health is well established the in ablebodied literature, specifically cardiovascular disease risk, this relationship is only beginning to emerge in the SCI population, as many traditional risk factors (i.e., waist circumference, blood pressure, and BMI) cannot be accurately applied. The greatest risk factors for death following SCI are heart disease and diabetes ${ }^{68}$ and it is further estimated that nearly one quarter of this population has metabolic syndrome and insulin resistance, ${ }^{8}$ all risk factors that can potentially be modified with lifestyle interventions.

Multiple studies have reported the efficacy of functional electrically stimulated (FES) leg cycling to improve glucose uptake and insulin sensitivity. ${ }^{69,70}$ This improvement has been attributed to changes in the expression of key regulatory proteins involved in glucose metabolism (i.e., GLUT 4) ${ }^{69,70}$ During periods of de-training, insulin sensitivity and 
GLUT 4 concentration return to baseline levels. ${ }^{70}$ Being physically active has further been linked to a reduction in total body mass, fat mass, C-reactive protein, insulin, and leptin, and more favourable profiles are associated with individuals who begin regular activity closer to the point of their injury. ${ }^{71}$

The relationship between aerobic exercise and indices of cardiovascular health are not as well established, although $\mathrm{VO}_{2 \text { peak }}$ has been shown to be predictive of insulin sensitivity, ${ }^{72}$ and exercise training protocols using either ACE or WCE have been shown to improve high density lipoprotein-cholesterol concentrations and reduce the total cholesterol/high density lipoprotein-cholesterol (TC/HDL-C) ratio. ${ }^{73,74}$

\subsection{Exercise Interventions to Improve Physical Fitness}

Several reviews and high quality randomized controlled trials support that exercise improves many aspects of fitness after SCI. ${ }^{75-77}$ The majority of these studies have focused on adults with chronic SCI. ${ }^{75}$

In terms of physical capacity, increases in $\mathrm{VO}_{2 \text { peak }}$ and $\mathrm{PO}_{\text {peak }}$ with training programs using either $\mathrm{ACE},{ }^{72,73,78-80} \mathrm{WCE},{ }^{74,81,82}$ or circuit training have been demonstrated ${ }^{83,84}$ While the magnitude of these improvements varies based on the training protocol used, one high quality randomized control trial demonstrated improvements of $81 \%$ in submaximal PO after 9-months of twice-weekly training on an ACE at $70 \%$ maximum heart rate. ${ }^{79}$ Using 
a WCE protocol of 45 minute exercise bouts 3 times per week, Bougenot et al.$^{82}$ revealed increases of $16 \%$ in $\mathrm{VO}_{2 \text { peak }}$ and $63 \%$ in PO. Circuit training, consisting of a combination of $\mathrm{ACE}$ and resistance training done in sequence, has been shown to produce increases of $29.7 \%$ in $\mathrm{VO}_{2 \text { peak }} \cdot{ }^{83}$ In one of the only studies on individuals with sub-acute SCI (mean $116 \pm 77$ days post-injury), de Groot et al..$^{72}$ reported training-induced increases of $150 \%$ and $117 \%$ in $\mathrm{VO}_{2 \text { peak }}$ in both a high (70-80\% $\mathrm{HR}$ reserve) and low (40-50\% HR reserve) intensity interval ACE training groups, respectively.

In regard to muscle strength, several studies have evaluated the effects of both traditional voluntary strength training and protocols using electrically stimulated exercise. Increases in upper body strength of 19-34\% have been reported following 9-months of twiceweekly strength training at $70-80 \%$ one repetition maximum (progressive) ${ }^{79}$ and 11.9$30 \%$ following 12 -weeks of circuit training 3 times per week. ${ }^{83}$ The benefits of neuromuscular electrical stimulation (NMES) and FES training are that they can be used to activate paralyzed muscle. Training programs using various modes of NMES and FESinduced exercise have shown to produce increases in quadriceps torque, fatigue resistance, and fibre area. ${ }^{85-87}$ 


\subsubsection{Exercise Prescription for Individuals with Spinal Cord Injury}

The changes in physical capacity and autonomic function create difficulties with prescribing exercise to persons with SCI, because traditional methods of prescribing exercise intensity cannot be applied. Further, the reliance on upper body exercise requires an alternative to traditional guidelines used for able-bodied population in both frequency and time of exercise.

Resting metabolic rate is reported as $14-27 \%$ lower among individuals with SCI compared to able-bodied controls, owing largely to a decrease in fat free mass and spontaneous physical activity. ${ }^{88-90}$ This decrease can lead to a positive energy balance and subsequent weight gain, obesity, and an increase in lifestyle-related disease risk. The baseline metabolic requirement of exercise (MET) has been reported as lower in the SCI population compared to the able-bodied population, with an estimate of $2.7 \mathrm{~mL} \cdot \mathrm{kg}^{-1} \cdot \mathrm{min}^{-1}$ compared to the standard $3.5 \mathrm{~mL} \cdot \mathrm{kg}^{-1} \cdot \mathrm{min}^{-1} .^{91}$ The energy cost of exercise has been shown to be greater using FES hybrid arm and leg exercise compared to using either arm or leg exercise alone, particularly among individuals with high level injuries..$^{92,93}$

The physical activity guidelines for adults with SCI were released in 2011 based on a systematic review of the evidence and an expert panel consensus meeting. ${ }^{75,94}$ The guidelines recommend exercise twice-weekly consisting of aerobic exercise at a moderate to vigorous intensity and strength training of 8-10 repetitions of each major muscle group. 


\subsection{Exercise Interventions using Behavioural Theory}

Several important theoretical constructs and subsequent interventions have been used to increase exercise participation among persons with SCI. Using the theory of planned behaviour, Latimer et al.$^{95}$ identified intentions as an important predictor of LTPA participation, explaining $16 \%$ of the variance. Intentions are a crucial element in the theory of planned behaviour and are influenced by one's attitudes, subjective norms (perceived social pressure to perform the behaviour), and perceived behavioural control (perceived ease or difficulty of performing the behaviour) ${ }^{96}$ Perceived behavioural control is also considered a co-determinant of behaviour, along with intentions. ${ }^{96}$ In order to encourage sustained LTPA participation, Latimer et al.$^{95}$ suggested interventions should bolster intentions with specific programs designed to highlight the benefit of regular exercise, encourage physicians or other health care professional to recommend exercise, and provide easy instructions on how to do certain exercises and adapt them to one's individual ability. Another way to strengthen the intention-behaviour relationship is the creation of implementation intentions, or action plans that identify when, where, and how a behaviour will be completed..$^{97}$ These action plans may be especially relevant to the SCI population given the numerous barriers to participation. Latimer et al.$^{98}$ demonstrated the efficacy of implementation intentions among a group of adults with SCI in completing a prescribed 30 minutes of moderate to heavy physical activity three times per week for 8 weeks. The intervention further improved measures of self-efficacy, intentions, and perceived control. ${ }^{98}$ A second aspect of planning, coping planning, involves the 
identification of anticipated barriers and self-regulatory strategies to overcome them. ${ }^{97}$ Interventions using this form of planning have been shown to be more effective in cardiac rehabilitation ${ }^{99}$ and in persons with SCI compared to action planning alone. ${ }^{100}$

Social cognitive theory is a theoretical framework that describes behaviour by means of a personal sense of control and that outlines perceived self-efficacy and outcome expectations as key constructs. ${ }^{101}$ Self-efficacy is defined as an individual's belief in his or her ability to perform a specific task or behaviour and can be enhanced through mastery experiences, vicarious experiences, and verbal persuasion. ${ }^{101}$ Outcome expectations are the other core construct and represent the beliefs about the consequences of a particular behaviour. ${ }^{101}$ This theory outlines self-efficacy as the strongest predictor of behaviour directly and indirectly through outcome expectations and self-regulation (goal setting and planning) ${ }^{102}$ In a systematic evaluation of social cognitive theory as a predictor of behaviour after SCI, Martin Ginis et al., ${ }^{103}$ found the model explained 39\% of the variance in LTPA behaviour, with self-regulation being the only significant and direct predictor. Other studies have identified self-efficacy beliefs as a significant variable in predicting both intensity and frequency of aerobic and strengthening exercise in persons with SCI. ${ }^{104}$ 


\section{Summary and Statement of Purpose}

Following an SCI, there are many immediate and substantial consequences pertaining to both health and fitness status that persist and worsen with increasing time post-injury. Physical activity trajectories for this population typically indicate decreasing participation with increasing time post-injury. This decrease in activity coincides with unfavourable trajectories of health status including increases in BMI, decreases in lean tissue mass, and decreases in physical capacity. Many of these changes can be favourably altered by exercise interventions; however, the effects appear to diminish following the cessation of training. ${ }^{70,105}$ This emphasises the need for long term, community-based interventions to encourage sustained participation. With a shift towards an increasing reliance on outpatient services for continued care of persons with $\mathrm{SCI},{ }^{22}$ there exists a great need for community exercise programming and protocols to compliment those services. A model recently proposed by Rimmer, ${ }^{25}$ suggested that, if exercise of sufficient dose is initiated near the end point of rehabilitation, individuals can maintain or improve upon health and functional gains achieved during rehabilitation. Further exploration of this concept has revealed a therapist-to-trainer model, wherein healthcare providers interact with exercise specialists during the transitional period at the end of rehabilitation to better formulate an exercise prescription and address salient barriers. ${ }^{106}$

The purpose of this dissertation is to explore the integration of structured exercise into the rehabilitation for persons with SCI and to optimize the exercise prescription as 
individuals reach a community setting. This will be completed through a series of four projects spanning the various stages of rehabilitation following SCI.

There is limited literature on exercise capacity or training status among individuals within the first year post injury. It is also not clear what exercise programming should involve in the early stages of rehabilitation and what intensity or exercise prescription might be appropriate. Further, since current $\mathrm{VO}_{2 \text { peak }}$ is the best predictor of future $\mathrm{VO}_{2 \text { peak }}$, evaluating peak physical capacity early in the rehabilitation process may assist with the development of rehabilitation goals. ${ }^{35}$ The purpose of the first study was to describe the aerobic capacity, orthostatic tolerance, and exercise perceptions of adults with sub-acute SCI. It was predicted that there would be differences in physical capacity and the prevalence of orthostatic hypotension based on level of injury and that participants would have favourable opinions of exercise when discharged from inpatient SCI rehabilitation.

Because of decreasing length of rehabilitation stays, the health care system is becoming increasingly reliant on outpatient services for the continued care of persons with SCI. ${ }^{19,22}$ Despite established benefits, there is currently very limited, if any, infrastructure in place for long-term exercise services and no decisive process in place by which to encourage exercise engagement post-discharge. ${ }^{19}$ The purpose of the second study was to evaluate the efficacy and feasibility of a direct referral process to community exercise following discharge from either inpatient or outpatient rehabilitation and to provide counselling 
support during this transitional period. It was predicted that this process would result in increased participation in regular exercise in the four months following discharge.

While evidence-based physical activity guidelines for adults with SCI have been developed, ${ }^{75,94}$ there are still several aspects of the exercise prescription for this population that are not clear. First, there is currently no mode of exercise, either aerobic or resistance, that has been identified as superior, nor is there any way to accurately prescribe exercise intensity to this population. Considering the considerable changes in autonomic function and functional ability, traditional prescription methods and modes of exercise used in the able-bodied literature cannot be applied. The purpose of this third phase study was to compare various types of adapted exercise equipment in terms of cardiovascular demand, energy expenditure, and consumer preference. It was predicted that persons with SCI would prefer hybrid (arm and leg) exercise modes and that this type of exercise would provide the greatest cardiovascular and metabolic challenge.

The purpose of the final study was to validate the physical activity guidelines for adults with SCI for improving aspects of fitness in a community setting. While these guidelines were developed from a robust evidence base and several training protocols have demonstrated increases in both aerobic and muscular fitness, ${ }^{72,75,79,80,82,84}$ their efficacy has yet to be established within a community-based setting. It was hypothesized that the physical activity guidelines would improve physical capacity and muscle strength following a 16-week intervention. 


\section{References}

1. Noonan VK, Fingas M, Farry A, Baxter D, Singh A, Fehlings MG, et al. Incidence and prevalence of spinal cord injury in Canada: a national perspective. Neuroepidemiology 2012;38:219-26.

2. The incidence and prevalence of spinal cord injury in Canada. Rick Hansen Institute; 2010.

3. Pickett GE, Campos-Benitez M, Keller JL, Duggal N. Epidemiology of traumatic spinal cord injury in Canada. Spine 2006;31:799-805.

4. DeVivo MJ. Epidemiology of traumatic spinal cord injury: trends and future implications. Spinal Cord 2012;50:365-72.

5. Maynard FM Jr, Bracken MB, Creasey G, Ditunno JF Jr, Donovan WH, Ducker $\mathrm{TB}$, et al. International standards for neurological and functional classification of spinal cord injury. Spinal Cord 1997;35:266.

6. Whiteneck GG, Charlifue SW, Frankel HL, Fraser MH, Gardner BP, Gerhart KA, et al. Mortality, morbidity, and psychosocial outcomes of persons spinal cord injured more than 20 years ago. Paraplegia 1992;30:617-30.

7. Groah SL, Nash MS, Ward EA, Libin A, Mendez AJ, Burns P, et al. Cardiometabolic risk in community-dwelling persons with chronic spinal cord injury. J Cardiopulm Rehabil Prev 2011;31:73-80.

8. Lee MY, Myers J, Hayes A, Madan S, Froelicher VF, Perkash I, et al. C-reactive protein, metabolic syndrome, and insulin resistance in individuals with spinal cord injury. J Spinal Cord Med 2005;28:20-5.

9. Spungen AM, Adkins RH, Stewart CA, Wang J, Pierson RN, Waters RL, et al. Factors influencing body composition in persons with spinal cord injury: a crosssectional study. J Appl Physiol 2003;95:2398-407.

10. Jones LM, Legge M, Goulding A. Healthy body mass index values often underestimate body fat in men with spinal cord injury. Arch Phys Med Rehabil 2003;84:1068-71.

11. Buchholz AC, McGillivray CF, Pencharz PB. The use of bioelectric impedance analysis to measure fluid compartments in subjects with chronic paraplegia. Arch Phys Med Rehabil 2003;84:854-61. 
12. Buchholz AC, Bugaresti JM. A review of body mass index and waist circumference as markers of obesity and coronary heart disease risk in persons with chronic spinal cord injury. Spinal Cord 2005;43:513-8.

13. Laughton GE, Buchholz AC, Ginis KAM, Goy RE. Lowering body mass index cutoffs better identifies obese persons with spinal cord injury. Spinal Cord 2009;47:757-62.

14. de Groot S, Post M, Postma K, Sluis TA, van der Woude L. Prospective analysis of body mass index during and up to 5 years after discharge from inpatient spinal cord injury rehabilitation. J Rehabil Med 2010;42:922-8.

15. de Groot S, Post MW, Snoek GJ, Schuitemaker M, van der Woude LH. Longitudinal association between lifestyle and coronary heart disease risk factors among individuals with spinal cord injury. Spinal Cord 2012;51:314-8.

16. Castro MJ, Apple DF Jr, Hillegass EA, Dudley GA. Influence of complete spinal cord injury on skeletal muscle cross-sectional area within the first 6 months of injury. Eur J Appl Physiol O 1999;80:373-8.

17. Castro MJ, Apple DF, Staron RS, Campos GE, Dudley GA. Influence of complete spinal cord injury on skeletal muscle within 6 mo of injury. J Appl Physiol 1999;86:350-8.

18. van den Berg-Emons RJ, Bussmann JB, Haisma JA, Sluis TA, van der Woude LH, Bergen MP, et al. A prospective study on physical activity levels after spinal cord injury during inpatient rehabilitation and the year after discharge. Arch Phys Med Rehabil 2008;89:2094-101.

19. Craven C, Verrier M, Balioussis C, Wolfe DL, J H, Noonan V, et al. Capturing capacity in Canadian SCI rehabilitation. Rick Hansen Institute; 2012.

20. Munce S, Wodchis WP, Guilcher SJ, Couris CM, Verrier M, Fung K, et al. Direct costs of adult traumatic spinal cord injury in Ontario. Spinal Cord 2012;

21. Ottenbacher KJ, Smith PM, Illig SB, Linn RT, Ostir GV, Granger CV. Trends in length of stay, living setting, functional outcome, and mortality following medical rehabilitation. JAMA 2004;292:1687-95.

22. Whiteneck GG, Gassaway J, Dijkers MP, Lammertse DP, Hammond F, Heinemann AW, et al. Inpatient and postdischarge rehabilitation services provided in the first year after spinal cord injury: findings from the SCIRehab study. Arch Phys Med Rehabil 2011;92:361-8. 
PhD Thesis - Chelsea A. Pelletier - McMaster University - Kinesiology

23. Scelza WM, Kirshblum SC, Wuermser L-A, Ho CH, Priebe MM, Chiodo AE. Spinal cord injury medicine. 4. community reintegration after spinal cord injury. Arch Phys Med Rehabil 2007;88:S71-5.

24. Tate DG, Forchheimer M. Enhancing community reintegration after inpatient rehabilitation for persons with spinal cord injury. Topics Spinal Cord Inj Rehabil 1998;4:42-55.

25. Rimmer JH. Getting Beyond the Plateau: Bridging the gap between rehabilitation and community-based exercise. PMRJ 2012;4:857-61.

26. Noreau L, Shephard RJ, Simard C, Paré G, Pomerleau P. Relationship of impairment and functional ability to habitual activity and fitness following spinal cord injury. Int J Rehabil Res 1993;16:265-75.

27. Dallmeijer AJ, van der Woude LHV. Health related functional status in men with spinal cord injury: relationship with lesion level and endurance capacity. Spinal Cord 2001;39:577-83.

28. Kilkens OJ, Dallmeijer AJ, Nene AV, Post MW, van der Woude LH. The longitudinal relation between physical capacity and wheelchair skill performance during inpatient rehabilitation of people with spinal cord injury. Arch Phys Med Rehabil 2005;86:1575-81.

29. Janssen TWJ, van Oers CAJM, Veeger HEJ, Hollander AP, van der Woude LHV, Rozendal RH. Relationship between physical strain during standardised ADL tasks and physical capacity in men with spinal cord injuries. Paraplegia 1994;32:844-59.

30. Noreau L, Shephard RJ. Return to work after spinal cord injury: the potential contribution of physical fitness. Paraplegia 1992;30:563-72.

31. Haisma JA, van der Woude LHV, Stam HJ, Bergen MP, Sluis TAR, Bussmann JBJ. Physical capacity in wheelchair-dependent persons with a spinal cord injury: a critical review of the literature. Spinal Cord 2006;44:642-52.

32. Janssen TWJ, Dallmeijer AJ, Veeger D, van der Woude LHV. Normative values and determinants of physical capacity in individuals with spinal cord injury. JRRD 2002;39:29-39.

33. Zoeller RF, Riechman SE, Dabayebeh IM, Goss FL, Robertson RJ, Jacobs PL. Relation between muscular strength and cardiorespiratory fitness in people with thoracic-level paraplegia. Arch Phys Med Rehabil 2005;86:1441-6. 
34. Haisma JA, Bussmann JB, Stam HJ, Sluis TA, Bergen MP, Dallmeijer AJ, et al. Changes in Physical Capacity During and After Inpatient Rehabilitation in Subjects With a Spinal Cord Injury. Arch Phys Med Rehabil 2006;87:741-8.

35. Haisma JA, van der Woude LH, Stam HJ, Bergen MP, Sluis TA, de Groot S, et al. Prognostic Models for Physical Capacity at Discharge and 1 Year Postdischarge From Rehabilitation in Persons With Spinal Cord Injury. Arch Phys Med Rehabil 2007;88:1694-703.

36. Haisma JA, Bussmann JBJ, Stam HJ, Sluis TAR, Bergen MP, Post MWM, et al. Physical fitness in people with a spinal cord injury: the association with complications and duration of rehabilitation. Clin Rehabil 2007;21:932-40.

37. Kim CM, Eng JJ, Whittaker MW. Level walking and ambulatory capacity in persons with incomplete spinal cord injury: relationship with muscle strength. Spinal Cord 2004;42:156-62.

38. Beninato M, O'Kane KS, Sullivan PE. Relationship between motor FIM and muscle strength in lower cervical-level spinal cord injuries. Spinal Cord 2004;42:533-40.

39. Giangregorio LM, Hicks AL, Webber CE, Phillips SM, Craven BC, Bugaresti JM, et al. Body weight supported treadmill training in acute spinal cord injury: impact on muscle and bone. Spinal Cord 2005;43:649-57.

40. Shah PK, Stevens JE, Gregory CM, Pathare NC, Jayaraman A, Bickel SC, et al. Lower-extremity muscle cross-sectional area after incomplete spinal cord injury. Arch Phys Med Rehabil 2006;87:772-8.

41. Round JM, Barr FM, Moffat B, Jones DA. Fibre areas and histochemical fibre types in the quadriceps muscle of paraplegic subjects. J Neurol Sci 1993;116:207-11.

42. Stewart BG, Tarnopolsky MA, Hicks AL, McCartney N, Mahoney DJ, Staron $\mathrm{RS}$, et al. Treadmill training-induced adaptations in muscle phenotype in persons with incomplete spinal cord injury. Muscle Nerve 2004;30:61-8.

43. Gorgey AS, Dudley GA. Skeletal muscle atrophy and increased intramuscular fat after incomplete spinal cord injury. Spinal Cord 2006;

44. Gorgey AS, Dudley GA. Spasticity may defend skeletal muscle size and composition after incomplete spinal cord injury. Spinal Cord 2007;46:96-102. 
PhD Thesis - Chelsea A. Pelletier - McMaster University - Kinesiology

45. Elder CP, Apple DF, Bickel CS, Meyer RA, Dudley GA. Intramuscular fat and glucose tolerance after spinal cord injury--a cross-sectional study. Spinal Cord 2004;42:711-6.

46. Burnham R, Martin T, Stein R, Bell G, MacLean I, Steadward R. Skeletal muscle fibre type transformation following spinal cord injury. Spinal Cord 1997;35:8691.

47. Gerrits HL, De Haan A, Hopman MT, van der Woude LH, Jones DA, Sargeant AJ. Contractile properties of the quadriceps muscle in individuals with spinal cord injury. Muscle Nerve 1999;22:1249-56.

48. Shields RK. Fatigability, relaxation properties, and electromyographic responses of the human paralyzed soleus muscle. J Neurophysiol 1995;73:2195-206.

49. Pelletier CA, Hicks AL. Muscle fatigue characteristics in paralyzed muscle after spinal cord injury. Spinal Cord 2010;49:125-30.

50. Pelletier CA, Hicks AL. The length-tension relationship of human dorsiflexor and plantarflexor muscles after spinal cord injury. Spinal Cord 2009;48:202-6.

51. Scott WB, Lee SC, Johnston TE, Binkley J, Binder-Macleod SA. Contractile properties and the force-frequency relationship of the paralyzed human quadriceps femoris muscle. Phys Ther 2006;86:788-99.

52. Jayaraman A, Gregory CM, Bowden M, Stevens JE, Shah P, Behrman AL, et al. Lower extremity skeletal muscle function in persons with incomplete spinal cord injury. Spinal Cord 2005;44:680-7.

53. Thomas CK, Zaidner EY, Calancie B, Broton JG, Bigland-Ritchie BR. Muscle weakness, paralysis, and atrophy after human cervical spinal cord injury. Exp Neurol 1997;148:414-23.

54. Claydon VE, Hol AT, Eng JJ, Krassioukov AV. Cardiovascular responses and postexercise hypotension after arm cycling exercise in subjects with spinal cord injury. Arch Phys Med Rehabil 2006;87:1106-14.

55. Myers JN, Hsu L, Hadley D, Lee MY, Kiratli BJ. Post-exercise heart rate recovery in individuals with spinal cord injury. Spinal Cord 2010;:1-6.

56. Krassioukov A. Autonomic function following cervical spinal cord injury. Resp Physiol Neurobiol 2009;169:157-64. 
57. The Consensus Committee of the American Autonomic Society and the American Academy of Neurology. Consensus statement on the definition of orthostatic hypotension, pure autonomic failure, and multiple system atrophy. Neurology 1996;46:1470-0.

58. Claydon VE, Krassioukov AV. Orthostatic Hypotension and autonomic pathways after spinal cord injury. J Neurotraum 2006;23:1713-25.

59. Claydon VE, Steeves JD, Krassioukov A. Orthostatic hypotension following spinal cord injury: understanding clinical pathophysiology. Spinal Cord 2005;44:341-51.

60. Sidorov EV, Townson AF, Dvorak MF, Kwon BK, Steeves J, Krassioukov A. Orthostatic hypotension in the first month following acute spinal cord injury. Spinal Cord 2007;46:65-9.

61. Sisto SA, Lorenz DJ, Hutchinson K, Wenzel L, Harkema SJ, Krassioukov A. Cardiovascular status of individuals with incomplete spinal cord injury from 7 NeuroRecovery Network rehabilitation centers. Arch Phys Med Rehabil 2012;93:1578-87.

62. Cowan RE, Nash MS, Anderson KD. Exercise participation barrier prevalence and association with exercise participation status in individuals with spinal cord injury. Spinal Cord 2013;51:27-32.

63. Vissers M, van den Berg-Emons R, Sluis T, Bergen M, Stam H, Bussmann H. Barriers to and facilitators of everyday physical activity in persons with a spinal cord injury after discharge from the rehabilitation centre. J Rehabil Med 2008;40:461-7.

64. Ginis KAM, Latimer AE, Arbour-Nicitopoulos KP, Buchholz AC, Bray SR, Craven BC, et al. Leisure time physical activity in a population-based sample of people with spinal cord injury part I: demographic and injury-related correlates. Arch Phys Med Rehabil 2010;91:722-8.

65. Anderson KD. Targeting recovery: priorities of the spinal cord-injured population. J Neurotraum 2004;21:1371-83.

66. Ginis KAM, Arbour-Nicitopoulos KP, Latimer AE, Buchholz AC, Bray SR, Craven BC, et al. Leisure time physical activity in a population-based sample of people with spinal cord injury part II: activity types, intensities, and durations. Arch Phys Med Rehabil 2010;91:729-33.

67. Scelza WM, Kalpakjian CZ, Zemper ED, Tate DG. Perceived barriers to exercise in people with spinal cord injury. Am J Phys Med Rehabil 2005;84:576-83. 
68. Garshick E, Kelley A, Cohen SA, Garrison A, Tun CG, Gagnon D, et al. A prospective assessment of mortality in chronic spinal cord injury. Spinal Cord 2005;43:408-16.

69. Hjeltnes N, Galuska D, Björnholm M, Aksnes AK, Lannem A, Zierath JR, et al. Exercise-induced overexpression of key regulatory proteins involved in glucose uptake and metabolism in tetraplegic persons: molecular mechanism for improved glucose homeostasis. FASEB J 1998;12:1701-12.

70. Mohr T, Dela F, Handberg A, Biering-Sørensen F, Galbo H, Kjaer M. Insulin action and long-term electrically induced training in individuals with spinal cord injuries. Med Sci Sports Exerc 2001;33:1247-52.

71. Koury JC, Passos MCF, Figueiredo FA, Chain A, Franco JG. Time of physical exercise practice after injury in cervical spinal cord-injured men is related to the increase in insulin sensitivity. Spinal Cord 2012;

72. de Groot PCE, Hjeltnes N, Heijboer AC, Stal W, Birkeland K. Effect of training intensity on physical capacity, lipid profile and insulin sensitivity in early rehabilitation of spinal cord injured individuals. Spinal Cord 2003;41:673-9.

73. El-Sayed MS, Younesian A. Lipid profiles are influenced by arm cranking exercise and training in individuals with spinal cord injury. Spinal Cord 2004;43:299-305.

74. Hooker SP, Wells CL. Effects of low- and moderate-intensity training in spinal cord-injured persons. Med Sci Sports Exerc 1989;21:18-21.

75. Hicks AL, Martin Ginis KA, Pelletier CA, Ditor DS, Foulon B, Wolfe DL. The effects of exercise training on physical capacity, strength, body composition and functional performance among adults with spinal cord injury: a systematic review. Spinal Cord 2011;49:1103-27.

76. Jacobs PL, Nash MS. Exercise recommendations for individuals with spinal cord injury. Sports Med 2004;34:727-51.

77. Devillard X, Rimaud D, Roche F, Calmels P. Effects of training programs for spinal cord injury. Annales de Réadaptation et de Médecine Physique. 2007;50:490-8.

78. Nilsson S, Staff PH, Pruett ED. Physical work capacity and the effect of training on subjects with long-standing paraplegia. Scand J Rehabil Med 1975;7:51. 
PhD Thesis - Chelsea A. Pelletier - McMaster University - Kinesiology

79. Hicks AL, Martin KA, Ditor DS, Latimer AE, Craven C, Bugaresti J, et al. Longterm exercise training in persons with spinal cord injury: effects on strength, arm ergometry performance and psychological well-being. Spinal Cord 2003;41:3443.

80. Bizzarini E, Saccavini M, Lipanje F, Magrin P, Malisan C, Zampa A. Exercise prescription in subjects with spinal cord injuries. Arch Phys Med Rehabil 2005;86:1170-5.

81. Gass GC, Watson J, Camp EM, McPherson LM, Redhead P. The effects of physical training on high level spinal lesion patients. Scand J Rehabil Med 1980;12:61.

82. Bougenot M-P, Tordi N, Betik AC, Martin X, Le Foll D, Parratte B, et al. Effects of a wheelchair ergometer training programme on spinal cord-injured persons. Spinal Cord 2003;41:451-6.

83. Jacobs PL, Nash MS, Rusinowski JW. Circuit training provides cardiorespiratory and strength benefits in persons with paraplegia. Med Sci Sports Exerc 2001;33:711-7.

84. Nash MS, van de Ven I, van Elk N, Johnson BM. Effects of circuit resistance training on fitness attributes and upper-extremity pain in middle-aged men with paraplegia. Arch Phys Med Rehabil 2007;88:70-5.

85. Sabatier MJ, Stoner L, Mahoney ET, Black C, Elder C, Dudley GA, et al. Electrically stimulated resistance training in SCI individuals increases muscle fatigue resistance but not femoral artery size or blood flow. Spinal Cord 2005;44:227-33.

86. Chilibeck PD, Jeon J, Weiss C, Bell G, Burnham R. Histochemical changes in muscle of individuals with spinal cord injury following functional electrical stimulated exercise training. Spinal Cord 1999;37:264-8.

87. Rodgers MM, Hooker P. Musculoskeletal responses of spinal cord injured individuals to functional neuromuscdar stimulation-induced knee extension exercise. JRRD 1991;28.

88. Buchholz AC, McGillivray CF, Pencharz PB. Differences in resting metabolic rate between paraplegic and able-bodied subjects are explained by differences in body composition. Am J Clin Nutr 2003;77:371-8. 
PhD Thesis - Chelsea A. Pelletier - McMaster University - Kinesiology

89. Monroe MB, Tataranni PA, Pratley R, Manore MM, Skinner JS, Ravussin E. Lower daily energy expenditure as measured by a respiratory chamber in subjects with spinal cord injury compared with control subjects. Am J Clin Nutr 1998;68:1223-7.

90. Buchholz AC, Pencharz PB. Energy expenditure in chronic spinal cord injury. Curr Opin Clin Nutr Metab Care. 2004;7:635-9.

91. Collins EG, Gater D, Kiratli J, Butler J, Hanson K, Langbein WE. Energy cost of physical activities in persons with spinal cord injury. Med Sci Sports Exerc 2010;42:691-700.

92. Hasnan N, Ektas N, Tanhoffer AIP, Tanhoffer R, Fornusek C, Middleton J, et al. Exercise Responses during FES Cycling in Individuals with Spinal Cord Injury. Med Sci Sports Exerc 2013;45:1131-8.

93. Brurok B, Tørhaug T, Karlsen T, Leivseth G, Helgerud J, Hoff J. Effect of lower extremity functional electrical stimulation pulsed isometric contractions on arm cycling peak oxygen uptake in spinal cord injured individuals. J Rehabil Med. 2013;45:254-9.

94. Ginis KAM, Hicks AL, Latimer AE, Warburton DER, Bourne C, Ditor DS, et al. The development of evidence-informed physical activity guidelines for adults with spinal cord injury. Spinal Cord 2011;49:1088-96.

95. Latimer AE, Martin Ginis KA. The theory of planned behavior in prediction of leisure time physical activity among individuals with Spinal Cord Injury. Rehabil Psychol 2005;50:389-96.

96. Ajzen I. The theory of planned behavior. Organizational behavior and human decision processes. 1991;50:179-211.

97. Sniehotta FF, Schwarzer R, Scholz U, Schüz B. Action planning and coping planning for long-term lifestyle change: theory and assessment. Eur J Soc Psychol. 2005;35:565-76.

98. Latimer AE, Ginis KAM, Arbour KP. The efficacy of an implementation intention intervention for promoting physical activity among individuals with spinal cord injury: A randomized controlled trial. Rehabil Psychol 2006;51:27380 .

99. Sniehotta FF, Scholz U, Schwarzer R. Action plans and coping plans for physical exercise: A longitudinal intervention study in cardiac rehabilitation. Brit J Health Psych 2010;11:23-37. 
PhD Thesis - Chelsea A. Pelletier - McMaster University - Kinesiology

100. Arbour-Nicitopoulos KP, Ginis KAM, Latimer AE. Planning, leisure-time physical activity, and coping self-efficacy in persons with spinal cord injury: A randomized controlled trial. Arch Phys Med Rehabil 2009;90:2003-11.

101. Conner M, Norman P. Predicting Health Behaviour. Open University Press; 2005.

102. Bandura A. Health promotion by social cognitive means. Health Educ Behav. 2004;31:143-64.

103. Ginis KAM, Latimer AE, Arbour-Nicitopoulos KP, Bassett RL, Wolfe DL, Hanna SE. Determinants of physical activity among people with spinal cord injury: a test of social cognitive theory. Ann Behav Med 2011;42:127-33.

104. Kroll T, Kratz A, Kehn M, Jensen MP, Groah S, Ljungberg IH, et al. Perceived exercise self-efficacy as a predictor of exercise behavior in individuals aging with spinal cord injury. Am J Phys Med Rehabil 2012;91:640-51.

105. Ditor DS, Latimer AE, Martin Ginis KA, Arbour KP, McCartney N, Hicks AL. Maintenance of exercise participation in individuals with spinal cord injury: effects on quality of life, stress and pain. Spinal Cord 2003;41:446-50.

106. Rimmer JH, Henley KY. Building the crossroad between inpatient/outpatient rehabilitation and lifelong community-based fitness for people with neurologic disability. JNPT 2013;37:72-77. 
Chapter 2

\title{
Aerobic Capacity, Orthostatic Tolerance, and Exercise Perceptions at Discharge from Inpatient Spinal Cord Injury Rehabilitation
}

\author{
Authors: Chelsea A. Pelletier, Graham Jones, Amy E. Latimer-Cheung, Darren E. \\ Warburton, Audrey L. Hicks
}

Published: Arch Phys Med Rehabil, 2013, doi:10.1016/j.apmr.2013.05.011

No reprint permission required. 
PhD Thesis - Chelsea A. Pelletier - McMaster University - Kinesiology

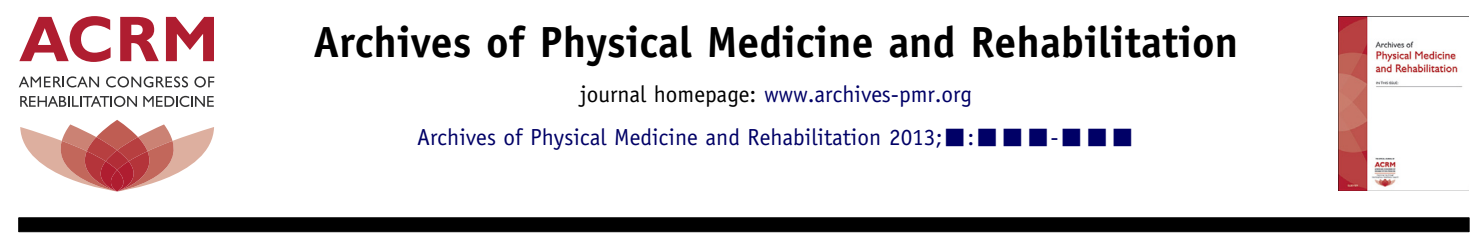

ORIGINAL ARTICLE

\title{
Aerobic Capacity, Orthostatic Tolerance, and Exercise Perceptions at Discharge From Inpatient Spinal Cord Injury Rehabilitation
}

\author{
Chelsea A. Pelletier, MSc, ${ }^{a}$ Graham Jones, PhD, MD, ${ }^{b}$ Amy E. Latimer-Cheung, $\mathrm{PhD}^{{ }^{c}}$ \\ Darren E. Warburton, PhD, ${ }^{d}$ Audrey L. Hicks, PhDa
}

From the ${ }^{a}$ Department of Kinesiology, McMaster University, Hamilton, ON; ${ }^{b}$ Department of Medicine, McMaster University, Hamilton, ON; ${ }^{c}$ Department of Kinesiology, Queen's University, Kingston, ON; and ${ }^{d}$ Experimental Medicine Program, Cardiovascular Physiology and Rehabilitation Laboratory, University of British Columbia, BC, Canada.

\begin{abstract}
Objective: To describe physical capacity, autonomic function, and perceptions of exercise among adults with subacute spinal cord injury (SCI). Design: Cross-sectional.

Setting: Two inpatient SCI rehabilitation programs in Canada.

Participants: Participants $(\mathrm{N}=41$; mean age $\pm \mathrm{SD}, 38.9 \pm 13.7 \mathrm{y})$ with tetraplegia $(\mathrm{TP} ; \mathrm{n}=19)$, high paraplegia $(\mathrm{HP}$; $\mathrm{n}=8)$, or low paraplegia $(\mathrm{LP} ; \mathrm{n}=14$ ) completing inpatient $\mathrm{SCI}$ rehabilitation (mean $\pm \mathrm{SD}, 112.9 \pm 52.5 \mathrm{~d}$ postinjury).

Interventions: Not applicable.

Main Outcome Measures: Peak exercise capacity was determined by an arm ergometry test. As a measure of autonomic function, orthostatic tolerance was assessed by a passive sit-up test. Self-efficacy for exercise postdischarge was evaluated by a questionnaire.

Results: There was a significant difference in peak oxygen consumption and heart rate between participants with $\mathrm{TP}\left(11.2 \pm 3.4 ; \mathrm{mL}^{\circ} \cdot \mathrm{kg}^{-1} \cdot \mathrm{min}^{-1}\right.$ $113.9 \pm 19.7 \mathrm{beats} / \mathrm{min})$ and $\mathrm{LP}\left(17.1 \pm 7.5 \mathrm{~mL} \cdot \mathrm{kg}^{-1} \cdot \mathrm{min}^{-1} ; 142.8 \pm 22.7\right.$ beats/min $)$. Peak power output was also significantly lower in the TP group $(30.0 \pm 6.9 \mathrm{~W})$ compared with the HP $(55.5 \pm 7.56 \mathrm{~W})$ and LP groups $(62.5 \pm 12.2 \mathrm{~W})$. Systolic blood pressure responses to the postural challenge varied significantly between groups $(-3.0 \pm 33.5 \mathrm{mmHg}$ in TP, $17.8 \pm 14.7 \mathrm{mmHg}$ in HP, $21.6 \pm 18.7 \mathrm{mmHg}$ in LP). Orthostatic hypotension was most prevalent among participants with motor complete TP $(73 \%)$. Results from the questionnaire revealed that although participants value exercise and see benefits to regular participation, they have low confidence in their abilities to perform the task of either aerobic or strengthening exercise.

Conclusions: Exercise is well tolerated in adults with subacute SCI. Exercise interventions at this stage should focus on improving task-specific self-efficacy, and attention should be made to blood pressure regulation, particularly in individuals with motor complete TP. Archives of Physical Medicine and Rehabilitation 2013;
\end{abstract}

(c) 2013 by the American Congress of Rehabilitation Medicine

It is well established that exercise can improve multiple aspects of physical fitness in adults with spinal cord injury (SCI). Because of the less stabilized nature of autonomic regulation and recovery in the first year postinjury, most research has focused on adults with

Supported by Rick Hansen Institute (grant no. 2010-80) and the Ontario Neurotrauma Foundation (grant no. 2009-RHI-MTNI-801).

No commercial party having a direct financial interest in the results of the research supporting this article has or will confer a benefit on the authors or on any organization with which the authors are associated. chronic SCI (>1y postinjury). ${ }^{1}$ Physical activity levels are typically reported as very low in the SCI population, ${ }^{2}$ with a significant decrease shown to occur in the months immediately after discharge from inpatient rehabilitation. ${ }^{3}$ This transitional period as patients reintegrate into the community may be an ideal time to introduce an exercise intervention in order to encourage sustained participation.

Aerobic capacity is often represented in terms of peak oxygen consumption $\left(\mathrm{VO}_{2 \text { peak }}\right)$ and has important implications for cardiovascular health, functional independence, and overall 
PhD Thesis - Chelsea A. Pelletier - McMaster University - Kinesiology

quality of life. ${ }^{4,5}$ Aerobic capacity is typically decreased after SCI because of a reliance on upper body exercise, decreased muscle mass, and a decrease in physical activity. Although clinical rehabilitation programs do provide a certain amount of cardiovascular strain, it is unknown if this stimulus is enough to improve measures of health-related physical fitness. ${ }^{6}$ Further, because the current physical activity guidelines are designed for adults with chronic SCI, ${ }^{7}$ the appropriate exercise prescription for adults $<1$ year postinjury is currently unknown. Given that $\mathrm{Vo}_{2 \text { peak }}$ and peak power output $\left(\mathrm{PO}_{\text {peak }}\right)$ are the strongest predictors of future physical capacity, ${ }^{8}$ accurate assessment of these outcomes early in the rehabilitation process may provide an indication of future prognosis and aid in the development of realistic rehabilitation goals together with an appropriate exercise prescription.

Autonomic dysfunction after SCI is caused by damage to autonomic pathways in the sympathetic nervous system and manifests as a decrease in cardiovascular control at rest and during exercise. ${ }^{9,10}$ Orthostatic hypotension $(\mathrm{OH})$ is defined as a decrease in systolic blood pressure (SBP) of $20 \mathrm{mmHg}$ or $10 \mathrm{mmHg}$ diastolic blood pressure (DBP) when moving to an upright position, regardless of symptoms. ${ }^{11}$ It is used as a clinical measure of autonomic function and is particularly prevalent among individuals in the acute phase of SCI and those with complete injuries above T6. ${ }^{10,12-14}$

In order to create a comprehensive intervention designed to promote the adoption of physical activity among individuals who are newly injured, it is imperative that we first gauge opinions of physical activity. Social cognitive theory provides a framework for behavior change and identifies self-efficacy and outcome expectations as important constructs for predicting behavior. ${ }^{15}$ In adults with chronic SCI, social cognitive theory has been shown to explain $39 \%$ of the variance in physical activity, ${ }^{16}$ and selfefficacy specifically has been related to the frequency and intensity of future aerobic and strengthening physical activity participation. ${ }^{17}$

The purpose of this study was to assess aerobic capacity, autonomic function, and perceptions about participating in regular physical activity among adults prior to being discharged from inpatient SCI rehabilitation. It was predicted that measures of cardiorespiratory and autonomic function would vary based on level of injury, with participants with tetraplegia (TP) showing the most pronounced decrease in $\mathrm{Vo}_{2 \text { peak }}, \mathrm{PO}_{\text {peak }}$, and peak heart rate, as well as having a greater propensity for orthostatic intolerance. Further, it was predicted that individuals with subacute SCI would demonstrate a willingness to engage in regular physical activity postdischarge and this would not vary significantly based on level of injury.

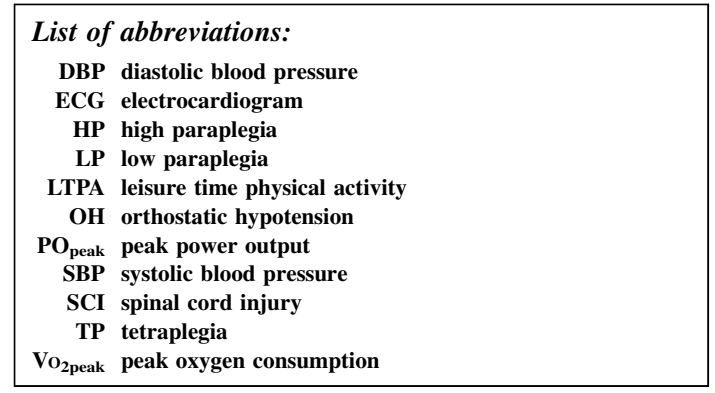

\section{Methods}

\section{Participants}

Participants were recruited from 2 inpatient SCI rehabilitation centers in Hamilton, Ontario, Canada, and Vancouver, British Columbia, Canada, from July 2010 to August 2012. Patients who were 18 to 65 years old, were manual wheelchair dependent, and could understand English were invited to participate in consultation with physiotherapists on their management team. Exclusion criteria included an active acute infection or dependence on ventilator support. Participants were classified according to level of injury: TP (C7 and above), high paraplegia (HP; T1-6), or low paraplegia (LP; T7 and below). These groups were chosen based on previous research and anticipated differences in autonomic and physical function. ${ }^{18,19}$ Written, informed consent was obtained from each participant and ethics approval was obtained from the relevant research ethics board at each study site.

\section{Peak exercise test}

Participants completed an incremental peak exercise test on an arm ergometer. ${ }^{\mathrm{a}}$

After a 1-minute warm-up, the resistance was increased every minute by $5 \mathrm{~W}$ for participants with TP and $10 \mathrm{~W}$ for participants with HP and LP. ${ }^{20}$ Participants were asked to continue arm cycling until volitional fatigue or they were unable to maintain a cadence of 40 revolutions per minute. All participants had experience using an arm ergometer, and tensor bandages were used to secure the hands of participants with insufficient grip strength. Expired gas and ventilatory parameters were acquired throughout the protocol using a metabolic cart that was calibrated before each test Continuous measurements of heart rate were also measured using an electrocardiogram (ECG). Ten-point ratings of perceived exertion were assessed every minute. ${ }^{21} \mathrm{Peak}_{\mathrm{VO}_{2}}$, peak heart rate, and peak minute ventilation were defined as the highest values recorded over 20-second averages. Peak PO was defined, in watts, as the greatest amount of resistance achieved during the arm bike protocol and maintained for at least 15 seconds.

\section{Sit-up test}

Continuous noninvasive blood pressure ${ }^{\mathrm{b}, \mathrm{c}}$ and 3-lead ECG monitoring was performed for 30 minutes during the procedure. After 15 minutes of supine rest, participants were passively moved into a seated position by raising the head of the bed to $90^{\circ}$ and by lowering the bottom portion of the bed below the knee to $90^{\circ}$. This position was maintained for 15 minutes. ${ }^{10}$ Change in SBP or DBP was calculated for each injury category and defined as the difference between mean seated and supine values $\left(\mathrm{BP}_{\text {change }}=\right.$ $\mathrm{BP}_{\text {seated }}-\mathrm{BP}_{\text {supine, }}$, where $\mathrm{BP}$ stands for blood pressure).

\section{Exercise Self-Efficacy Questionnaire}

Participants were asked to complete a questionnaire to measure individual perceptions and opinions about engaging in regular leisure time physical activity (LTPA) in the next 3 months postdischarge. LTPA was defined as activities that require exertion that individuals choose to do in their free time. This questionnaire 
PhD Thesis - Chelsea A. Pelletier - McMaster University - Kinesiology

\begin{tabular}{lllll} 
Table 1 Participant characteristics & & & \\
\hline Characteristic & Overall & TP & HP & LP \\
\hline $\mathrm{n}$ & 41 & 19 & 8 & 14 \\
Age (y) & $38.9 \pm 13.7$ & $40.5 \pm 15.6$ & $42.4 \pm 10.9$ & $34.7 \pm 12.2$ \\
Sex (males/females) & $27 / 14$ & $14 / 5$ & $6 / 2$ & $7 / 7$ \\
BMI & $25.2 \pm 5.5$ & $24.5 \pm 4.9$ & $26.9 \pm 5.7$ & $25.3 \pm 6.4$ \\
Level of injury & C3-L5 & C3-7 & T3-6 & T7-L5 \\
AIS score & & 11 & 5 & 9 \\
$\quad$ Motor complete (grades A and B) & 25 & 8 & 3 & 5 \\
$\quad$ Motor incomplete (grades C and D) & 16 & $128.4 \pm 62.1$ & $104.0 \pm 44.1$ & $99.3 \pm 41.4$ \\
Time postinjury (d) & $112.9 \pm 52.5$ & $79.3 \pm 30.2$ & $57.4 \pm 21.4$ & $67.2 \pm 26.2$ \\
Time in rehabilitation (d) & $70.6 \pm 27.9$ & & &
\end{tabular}

was adapted from measures previously used in the SCI population ${ }^{16,22,23}$ to be relevant to patients in this stage of rehabilitation.

All self-efficacy items were rated on a 7-point scale from 1 (not at all confident) to 7 (completely confident). Task self-efficacy was measured using 4 scales (moderate and heavy intensity aerobic and strengthening activity), and participants were asked to rate their confidence to engage in each activity for $10,20,30,45$, and 60 minutes without stopping. ${ }^{16}$ Scheduling self-efficacy was measured by asking participants to rate their confidence in their ability to include 30 minutes of LTPA into their schedule 1, 2, 3, and $>3$ times per week. ${ }^{22,23}$ Internal consistency was good for all self-efficacy measures $\left(\alpha_{\text {aerobic }}=0.91, \alpha_{\text {strengthening }}=0.95, \alpha_{\text {scheduling }}=0.86\right) .{ }^{24}$

Outcome value and outcome expectations were also rated on a 7-point scale from 1 (completely disagree) to 7 (completely agree). Outcome value had good internal consistency $(\alpha=.86)^{24}$ and was measured with 3 items: in the next 3 months postdischarge, engaging in regular LTPA is the most important thing I can do; establishing a routine including regular LTPA in the next 3 months postdischarge is a high priority for me; and engaging in regular LTPA postdischarge would be extremely valuable. Outcome expectations were measured with 12 items that were preceded with the statement: "I think that engaging in regular LTPA in the next 3 months postdischarge will..." Then participants were asked to rate various health and lifestyle outcomes specific to $\mathrm{SCI}{ }^{16}$ Internal consistency was adequate $(\alpha=.76){ }^{24}$

\section{Data analysis}

All data are presented as means \pm SDs. Statistical analysis was completed using IBM SPSS Statistics, Version 20. ${ }^{\mathrm{d}}$ Data were evaluated and met assumptions for normality and equal variances using the Shapiro-Wilk test and Levene statistic, respectively. Group means were compared using a 1-way analysis of variance and Tukey post hoc tests where appropriate. Further analysis was performed within each injury category using a paired $t$ test. Significance was accepted at $P<.05$.

\section{Results}

\section{Participants}

Forty-one participants with subacute SCIs were recruited during inpatient rehabilitation. Participant characteristics are summarized in table 1 . The most common cause of injury was motor vehicle collisions (36.6\%), sport or recreation accidents (21.9\%), and falls (19.5\%), which are similar to nationally reported values for the given age group $(38.9 \pm 13.7 \mathrm{y}){ }^{25}$ Most participants were men $(65.8 \%)$. Time in rehabilitation varied between groups, because participants with higher injuries were enrolled for longer (79.3 $\pm 30.3 \mathrm{~d}$ in TP; $57.4 \pm 21.4 \mathrm{~d}$ in HP; $67.2 \pm 26.2 \mathrm{~d}$ in LP). This is also reflected when examining group differences in time postinjury as participants with TP had the longest duration of injury (128.4 $\pm 62.1 \mathrm{~d})$, followed by those with HP $(104 \pm 44.1 \mathrm{~d})$ and LP ( $99.3 \pm 41.4 \mathrm{~d})$; however, because of the high degree of individual variability, these differences were not significant

\section{Peak exercise test}

Thirty-eight participants completed the peak exercise test, and there were no adverse exercise-related events or abnormal ECG findings. Three participants declined participation in this portion of the protocol. Results are summarized in table 2. Relative and absolute $\mathrm{VO}_{2 \text { peak }}$ values were significantly different between $\mathrm{TP}$ and LP groups ( $P=.02$ and $P=.01$, respectively), as were peak heart rate values $(P=.00)$. Peak $\mathrm{PO}$ varied significantly between the TP and HP groups $(P=.00)$ and the TP and LP groups $(P=.00)$. Mean peak respiratory exchange ratio values $>1.0$ in all groups and mean 10-point ratings of perceived exertion values $(8.2 \pm 1.76$ in $\mathrm{TP}, 8.4 \pm 1.30$ in $\mathrm{HP}, 9.1 \pm 1.33$ in LP) indicate that participants were able to achieve maximal effort regardless of injury level.

\section{Orthostatic tolerance}

There were 37 participants who completed the passive sit-up test and 4 participants who declined participation. Among participants with TP, 9 (50\%) experienced $\mathrm{OH}$, but for those with motor complete TP (ASIA Impairment Scale grade A or B), this rate increased to $73 \%$. In most cases (6 out of 9), the decrease in SBP or DBP was accompanied with common symptoms of lightheadedness, dizziness, and/or vision changes. In the case of 4 participants, the test was terminated early because of symptoms, and the participant was returned to the supine position. In the HP group, 2 (29\%) participants experienced a decreased SBP or DBP, indicative of $\mathrm{OH}$, but neither was associated with any reported symptoms. In the $\mathrm{LP}$ group, 1 participant experienced $\mathrm{OH}$, and the test was terminated because of symptoms. The prevalence of $\mathrm{OH}$ based on level and severity of injury is presented in table 3 . 
PhD Thesis - Chelsea A. Pelletier - McMaster University - Kinesiology

\begin{tabular}{|c|c|c|c|}
\hline Variable & TP & HP & LP \\
\hline $\begin{array}{l}\text { Time to peak } \\
\text { (min:s) }\end{array}$ & $6: 07 \pm 2: 35$ & $6: 08 \pm 0: 55$ & $7: 13 \pm 0: 55$ \\
\hline $\begin{array}{l}\mathrm{V}_{\mathrm{O}_{\text {2peak }}} \\
\quad\left(\mathrm{mL} \cdot \mathrm{kg}^{-1} \cdot \mathrm{min}^{-1}\right)\end{array}$ & $11.23 \pm 3.39$ & $15.23 \pm 4.82$ & $17.09 \pm 7.50^{*}$ \\
\hline $\mathrm{V}_{0_{\text {2peak }}}(\mathrm{L} / \mathrm{min})$ & $0.82 \pm 0.27$ & $1.22 \pm 0.31$ & $1.20 \pm 0.45^{*}$ \\
\hline $\begin{array}{l}\mathrm{HR}_{\text {peak }} \\
\text { (beats } / \mathrm{min} \text { ) }\end{array}$ & $113.94 \pm 19.68$ & $130.14 \pm 20.75$ & $142.77 \pm 22.71^{*}$ \\
\hline $\mathrm{RER}_{\text {peak }}$ & $1.17 \pm 0.24$ & $1.18 \pm 0.24$ & $1.16 \pm 0.25$ \\
\hline $\mathrm{RPE}_{\text {peak }}$ & $8.19 \pm 1.76$ & $8.38 \pm 1.30$ & $9.07 \pm 1.33$ \\
\hline$V_{E_{\text {peak }}}(\mathrm{L} / \mathrm{min})$ & $31.99 \pm 12.97$ & $39.53 \pm 11.23$ & $41.38 \pm 14.22$ \\
\hline $\mathrm{PO}_{\text {peak }}(\mathrm{W})$ & $30.00 \pm 16.93$ & $55.00 \pm 7.56^{\dagger}$ & $62.50 \pm 12.21^{*}$ \\
\hline
\end{tabular}

NOTE. Values are mean \pm SD.

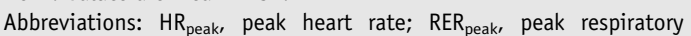
exchange ratio; $R P E_{\text {peak }}$, peak rating of perceived exertion (10-point scale); $V_{E_{\text {peak }}}$ peak minute ventilation.

* Significant difference between the TP group and LP group.

$\dagger$ Significant difference between the TP group and HP group.

Overall, there was a significant increase in SBP in the HP $(P=.02)$ and $\mathrm{LP}(P=.00)$ groups when moving from a supine to seated position, whereas the TP group had a decrease in SBP $(-3.0 \pm 33.5 \mathrm{mmHg})$. Increases in DBP were significant in the TP $(P=.05)$, HP $(P=.01)$, and LP $(P=.00)$ groups. Blood pressure changes during the protocol are represented graphically in figure 1 .

\section{Group differences in blood pressure and heart rate}

There were no significant group differences in supine SBP. In the seated position, $\mathrm{SBP}$ was significantly greater in both the $\mathrm{HP}(P=.00)$ and LP groups $(P=.01)$ compared with participants with TP.

There were also no significant differences in supine DBP between groups. Seated values were significantly lower in the TP group compared with the HP $(P=.02)$ group, but not the LP group $(P=.08)$.

Table 3 Prevalence of $\mathrm{OH}$ based on level and severity of injury

\begin{tabular}{|c|c|c|c|}
\hline & $\begin{array}{l}\text { Cases of } \mathrm{OH} \\
\text { (n/total sample) }\end{array}$ & $\begin{array}{l}\text { SBP Change } \\
(\mathrm{mmHg})\end{array}$ & $\begin{array}{l}\text { DBP Change } \\
(\mathrm{mmHg})\end{array}$ \\
\hline \multicolumn{4}{|l|}{ TP } \\
\hline Motor complete & $8 / 11$ & $-18.7 \pm 34.9$ & $3.3 \pm 15.4$ \\
\hline Motor incomplete & $1 / 7$ & $21.7 \pm 15.5^{*}$ & $17.4 \pm 16.9^{*}$ \\
\hline Overall & $9 / 18$ & $-3.0 \pm 33.5$ & $8.8 \pm 16.3^{*}$ \\
\hline \multicolumn{4}{|l|}{ HP } \\
\hline Motor complete & $1 / 4$ & $16.9 \pm 19.7$ & $20.2 \pm 15.7$ \\
\hline Motor incomplete & $1 / 3$ & $18.9 \pm 8.5$ & $18.1 \pm 9.6$ \\
\hline Overall & $2 / 7$ & $17.8 \pm 14.7^{*}$ & $19.3 \pm 12.4^{*}$ \\
\hline \multicolumn{4}{|l|}{ LP } \\
\hline Motor complete & $0 / 7$ & $19.3 \pm 11.2^{*}$ & $20.1 \pm 7.1^{*}$ \\
\hline Motor incomplete & $1 / 5$ & $24.9 \pm 27.3$ & $17.7 \pm 18.2$ \\
\hline Overall & $1 / 12$ & $21.6 \pm 18.7^{*}$ & $19.1 \pm 12.2^{*}$ \\
\hline
\end{tabular}
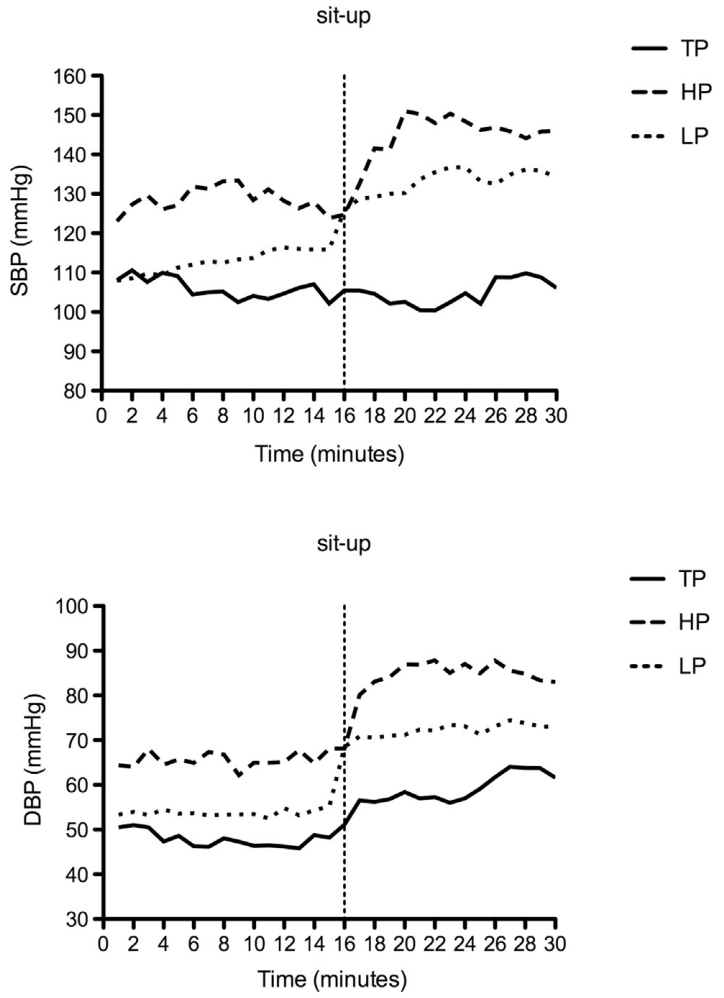

Fig 1 Mean SBP and DBP changes during a 30-minute sit-up test in participants with TP, HP, and LP.

There were no significant group differences in heart rate in either the supine or seated position. There was a significant increase in heart rate in the TP $(P=.00)$ and LP $(P=.04)$ groups during the protocol. Changes in SBP, DBP, and heart rate before and after the postural challenge are presented in table 4 .

\section{Exercise Self-Efficacy Questionnaire}

Forty-one participants completed the Exercise Self-Efficacy Questionnaire. Mean composite scores are presented out of a maximum of 7 (completely agree or completely confident) and are summarized in table 5. Overall, the values for outcome value (5.9 \pm 1.12$)$, outcome expectations (5.6 \pm .77$)$, and scheduling selfefficacy $(5.7 \pm 1.28)$ indicate that participants in all groups agree with the respective statements about regular physical activity. Task self-efficacy values were relatively lower for both aerobic $(4.0 \pm 1.20)$ and strengthening (4.2 \pm 1.57$)$ activities. The only significant group difference appeared in the measure of outcome expectations, with the HP group demonstrating a significantly lower mean composite score compared with the LP group $(P=.046)$.

\section{Discussion}

A novel aspect of the present study was to examine patients' perceptions of their ability to participate in structured exercise on 
PhD Thesis - Chelsea A. Pelletier - McMaster University - Kinesiology

\begin{tabular}{|c|c|c|}
\hline & Supine & Seated \\
\hline \multicolumn{3}{|c|}{$\mathrm{SBP}(\mathrm{mmHg})$} \\
\hline TP & $105.5 \pm 22.2$ & $102.5 \pm 21.6$ \\
\hline HP & $127.9 \pm 86.8$ & $145.7 \pm 40.3^{*_{+}}$ \\
\hline LP & $112.4 \pm 24.0$ & $133.9 \pm 25.9^{* \dagger}$ \\
\hline \multicolumn{3}{|c|}{$\mathrm{DBP}(\mathrm{mmHg})$} \\
\hline TP & $48.0 \pm 13.5$ & $56.8 \pm 18.7^{*}$ \\
\hline HP & $65.8 \pm 20.7$ & $85.1 \pm 30.1^{* \dagger}$ \\
\hline LP & $55.5 \pm 10.7$ & $74.6 \pm 18.3^{*}$ \\
\hline \multicolumn{3}{|c|}{ Heart rate (beats/min) } \\
\hline TP & $69.5 \pm 13.7$ & $79.3 \pm 14.1^{*}$ \\
\hline HP & $74.4 \pm 9.4$ & $80.5 \pm 13.3$ \\
\hline LP & $79.1 \pm 9.1$ & $86.1 \pm 11.9^{*}$ \\
\hline
\end{tabular}

discharge from rehabilitation, in addition to evaluating cardiovascular parameters associated with exercise performance. Our findings indicate that although there may be significant cardiovascular detraining and changes in autonomic regulation that occur secondary to SCI, ${ }^{10,19,26}$ these changes should not interfere with regular exercise participation or prescription. Further, in agreement with our hypothesis, there was a significant difference in $\mathrm{VO}_{2 \text { peak }}$, peak heart rate, and $\mathrm{PO}_{\text {peak }}$ between adults with $\mathrm{TP}$ and adults with LP. OH was also more prevalent among adults with TP, and there were significant differences in blood pressure responses to the passive postural challenge based on level and completeness of injury.

Several reports of physical capacity in individuals with chronic SCI indicate that cardiorespiratory fitness is not considerably different than in individuals with subacute SCI in the current study. ${ }^{19,20,27}$ According to normative $\mathrm{Vo}_{2 \text { peak }}$ values based on the level of injury for the general SCI population, the mean group averages from the current study would be classified as average $\left(10.01-13.39 \mathrm{~mL} \cdot \mathrm{kg}^{-1} \cdot \mathrm{min}^{-1}\right)$ for the TP group, and fair $(16.51-$ $22.70 \mathrm{~mL} \cdot \mathrm{kg}^{-1} \cdot \mathrm{min}^{-1}$ ) for the HP and LP groups. ${ }^{28}$ In a longitudinal study of physical capacity over the first year postinjury, Haisma et al ${ }^{29}$ showed progressive increases in $\mathrm{VO}_{2 \text { peak }}$ and $\mathrm{PO}_{\text {peak }}$ in participants with TP but not those with paraplegia. At discharge, $\mathrm{VO}_{2 \text { peak }}$ values were higher than what was observed in

Table 5 Mean composite scores for outcome value, outcome expectation, scheduling, and task self-efficacy

\begin{tabular}{|c|c|c|c|}
\hline Scale & TP & HP & LP \\
\hline Outcome value & $5.9 \pm 1.23$ & $5.5 \pm 1.07$ & $6.1 \pm 0.99$ \\
\hline Outcome expectations & $5.7 \pm 0.72$ & $5.0 \pm 0.96$ & $5.8 \pm 0.58^{*}$ \\
\hline Scheduling self-efficacy & $6.0 \pm 1.06$ & $5.7 \pm 1.32$ & $5.3 \pm 1.48$ \\
\hline $\begin{array}{l}\text { Task self-efficacy: } \\
\text { aerobic exercise }\end{array}$ & $4.0 \pm 1.19$ & $4.5 \pm 1.09$ & $3.8 \pm 1.30$ \\
\hline $\begin{array}{l}\text { Task self-efficacy: } \\
\text { strengthening exercise }\end{array}$ & $3.9 \pm 1.75$ & $4.6 \pm 0.88$ & $4.2 \pm 1.63$ \\
\hline
\end{tabular}

the current study, with values of $0.99 \mathrm{~L} / \mathrm{min}$ in participants with $\mathrm{TP}$, and $1.32 \mathrm{~L} / \mathrm{min}$ in participants with paraplegia; however, this is likely because of the longer duration of inpatient rehabilitation in the previous work. ${ }^{29}$ An increase in aerobic capacity after rehabilitation may be representative of an increase in upper limb function and muscle strength, particularly among individuals with TP. Most participants in the current study indicated the reason they stopped the exercise test was because of arm muscle fatigue. Given the relationship between muscle strength and cardiorespiratory fitness in this population, ${ }^{30}$ it may be prudent to emphasize upper body muscle strength throughout the rehabilitation process.

Our assessment of orthostatic tolerance reveals that $\mathrm{OH}$ is still a considerable issue at the end of the formal rehabilitation process for people with cervical SCI. Among individuals with TP, $50 \%$ of participants had blood pressure decreases or symptoms indicative of $\mathrm{OH}$. Among participants with motor complete $\mathrm{TP}$, the rate increased to $73 \%$. These values are similar to what is seen in the chronic and acute (1 month postinjury) SCI population. $^{10,12}$ Among individuals with HP, $29 \%$ of participants experienced clinical $\mathrm{OH}$, with roughly similar rates between those with motor complete (25\%) and incomplete (33\%) injuries. This is slightly higher than what has been shown previously, ${ }^{10,12,13}$ although the relatively small number of participants in this group $(n=8)$ limits the interpretation of the findings.

Resting blood pressure values and responses to the passive situp test in the present study are also relatively similar to a sample of individuals with chronic SCI, ${ }^{10}$ although reports of participants with motor incomplete SCI show consistently higher SBP and DBP values across all groups. ${ }^{13}$

The self-efficacy questionnaire indicated that participants value exercise at this stage of rehabilitation and they perceive many benefits to regular participation. Although participants rated high levels of scheduling self-efficacy, task self-efficacy values showed room for improvement. ${ }^{22}$ This may indicate that participants are not confident in their ability to perform the physical task of aerobic and strengthening activities, regardless of level of injury. This is not entirely unexpected given the relatively short time postinjury and limited experience with adapting exercise to accommodate their abilities. Using social cognitive theory as a framework, interventions should be designed to increase taskspecific self-efficacy and include specific information on how to complete various exercises as well as exposure to peers with a similar disability status participating in exercise. ${ }^{15}$ One other significant variable in social cognitive theory that is closely implicated with future LTPA participation in the SCI population is self-regulation, a measure of one's ability to control specific behaviors. ${ }^{16}$ We did not feel this measure would be appropriate given the rehabilitation status of our participants; however, interventions that incorporate action and coping planning have proven to be effective among adults with chronic SCI, and after discharge from cardiac rehabilitation. ${ }^{22,31}$ Given that the months after discharge are highly transitional as individuals work to establish a new routine, it may be a critical time to introduce and encourage new habits that incorporate regular LTPA.

\section{Study limitations}

One of the limitations of this study was our uneven group size, which is the result of our convenience sample method of recruitment. This limits the generalizability to the larger SCI population. Further work should explore longitudinal assessment 
PhD Thesis - Chelsea A. Pelletier - McMaster University - Kinesiology

of cardiovascular and autonomic function throughout the rehabilitation process and beyond discharge.

\section{Conclusions}

To our knowledge, this is the first study to combine a physiological assessment of cardiorespiratory fitness and autonomic function with an evaluation of psychological willingness and perceptions for exercise among adults with subacute SCI. The results of this study support the inclusion of regular structured exercise during SCI rehabilitation and in the months after discharge, because physical capacity does not appear to be considerably different than what is observed among individuals with longstanding injuries. Physical activity interventions should be designed with special caution of orthostatic tolerance and provide patients with additional counseling on how to adapt exercises to accommodate their abilities.

\section{Suppliers}

a. Monark Arm Ergometer (Rehab Trainer) and Adjustable Height Table; Patterson Medical Supply Inc, 755 Queensway E, Unit 27, Mississauga, ON, Canada, L4Y 4C5.

b. Finometer Model-2 s/n 100205193; Finapres Medical Systems BV, Paasheuvelweg 34a, 1105 BJ Amsterdam, The Netherlands.

c. PowerLab 4/25, SN 425-0048, ML845; AD Instruments, Inc, 2205 Executive Circle, Colorado Springs, CO 80906.

d. IBM SPSS Statistics, Version 20.0; SPSS Inc., 233 S Wacker Dr, Chicago, IL 60606.

\section{Keywords}

Exercise; Hypotension, orthostatic; Rehabilitation; Self efficacy; Spinal cord injuries

\section{Corresponding author}

Audrey L. Hicks, PhD, Department of Kinesiology, McMaster University, 1280 Main St W, Hamilton, ON, L8S 4K1, Canada. E-mail address: hicksal@mcmaster.ca.

\section{References}

1. Hicks AL, Martin Ginis KA, Pelletier CA, Ditor DS, Foulon B, Wolfe DL. The effects of exercise training on physical capacity, strength, body composition and functional performance among adults with spinal cord injury: a systematic review. Spinal Cord 2011;49: 1103-27.

2. Ginis KA, Latimer AE, Arbour-Nicitopoulos KP, et al. Leisure time physical activity in a population-based sample of people with spinal cord injury part I: demographic and injury-related correlates. Arch Phys Med Rehabil 2010;91:722-8.

3. van den Berg-Emons RJ, Bussmann JB, Haisma JA, et al. A prospective study on physical activity levels after spinal cord injury during inpatient rehabilitation and the year after discharge. Arch Phys Med Rehabil 2008;89:2094-101

4. Dallmeijer AJ, van der Woude LH. Health related functional status in men with spinal cord injury: relationship with lesion level and endurance capacity. Spinal Cord 2001;39:577-83.
5. Noreau L, Shephard RJ, Simard C, Paré G, Pomerleau P. Relationship of impairment and functional ability to habitual activity and fitness following spinal cord injury. Int J Rehabil Res 1993;16: $265-75$.

6. Koopman AD, Eken MM, van Bezeij T, Valent LJ, Houdijk H. Does clinical rehabilitation impose sufficient cardiorespiratory strain to improve aerobic fitness? J Rehabil Med 2013;45:92-8.

7. Ginis KA, Hicks AL, Latimer AE, et al. The development of evidenceinformed physical activity guidelines for adults with spinal cord injury. Spinal Cord 2011;49:1088-96.

8. Haisma JA, van der Woude LH, Stam HJ, et al. Prognostic models for physical capacity at discharge and 1 year postdischarge from rehabilitation in persons with spinal cord injury. Arch Phys Med Rehabil 2007;88:1694-703.

9. Claydon VE, Hol AT, Eng JJ, Krassioukov AV. Cardiovascular responses and postexercise hypotension after arm cycling exercise in subjects with spinal cord injury. Arch Phys Med Rehabil 2006;87: 1106-14.

10. Claydon VE, Krassioukov AV. Orthostatic hypotension and autonomic pathways after spinal cord injury. J Neurotrauma 2006;23 1713-25.

11. The Consensus Committee of the American Autonomic Society and the American Academy of Neurology. Consensus statement on the definition of orthostatic hypotension, pure autonomic failure, and multiple system atrophy. Neurology 1996;46:1470.

12. Sidorov EV, Townson AF, Dvorak MF, Kwon BK, Steeves J, Krassioukov A. Orthostatic hypotension in the first month following acute spinal cord injury. Spinal Cord 2007;46:65-9.

13. Sisto SA, Lorenz DJ, Hutchinson K, Wenzel L, Harkema SJ, Krassioukov A. Cardiovascular status of individuals with incomplete spinal cord injury from 7 NeuroRecovery Network rehabilitation centers. Arch Phys Med Rehabil 2012;93: 1578-87.

14. Krassioukov A, Biering-Sørensen F, Donovan W, et al. International standards to document remaining autonomic function after spinal cord injury. J Spinal Cord Med 2012;35:201-10.

15. Bandura A. Health promotion by social cognitive means. Health Educ Behav 2004;31:143-64.

16. Ginis KA, Latimer AE, Arbour-Nicitopoulos KP, Bassett RL, Wolfe DL, Hanna SE. Determinants of physical activity among people with spinal cord injury: a test of social cognitive theory. Ann Behav Med 2011;42:127-33.

17. Kroll T, Kratz A, Kehn M, et al. Perceived exercise self-efficacy as a predictor of exercise behavior in individuals aging with spinal cord injury. Am J Phys Med Rehabil 2012;91:640-51.

18. Krassioukov A. Autonomic function following cervical spinal cord injury. Respir Physiol Neurobiol 2009;169:157-64.

19. Myers JN, Hsu L, Hadley D, Lee MY, Kiratli BJ. Post-exercise heart rate recovery in individuals with spinal cord injury. Spinal Cord 2010 48:639-44.

20. Hol AT, Eng JJ, Miller WC, Sproule S, Krassioukov AV. Reliability and validity of the six-minute arm test for the evaluation of cardiovascular fitness in people with spinal cord injury. Arch Phys Med Rehabil 2007;88:489-95.

21. Borg G. Psychophysical bases of perceived exertion. Med Sci Sports Exerc 1982;14:377-81.

22. Arbour-Nicitopoulos KP, Ginis KA, Latimer AE. Planning, leisuretime physical activity, and coping self-efficacy in persons with spinal cord injury: a randomized controlled trial. Arch Phys Med Rehabil 2009;90:2003-11.

23. Latimer AE, Ginis KA, Arbour KP. The efficacy of an implementation intention intervention for promoting physical activity among individuals with spinal cord injury: a randomized controlled trial. Rehabil Psychol 2006;51:273-80.

24. Nunnally JC. Psychometric theory. 2nd ed. New York: McGraw-Hill; 1978.

25. Pickett GE, Campos-Benitez M, Keller JL, Duggal N. Epidemiology of traumatic spinal cord injury in Canada. Spine 2006;31:799-805. 
PhD Thesis - Chelsea A. Pelletier - McMaster University - Kinesiology

26. Van Loan MD, McCluer S, Loftin JM, Boileau RA. Comparison of physiological responses to maximal arm exercise among able-bodied, paraplegics and quadriplegics. Paraplegia 1987;25:397-405.

27. Haisma JA, van der Woude LH, Stam HJ, Bergen MP, Sluis TAR, Bussmann JB. Physical capacity in wheelchair-dependent persons with a spinal cord injury: a critical review of the literature. Spinal Cord 2006; 44:642-52.

28. Janssen TW, Dallmeijer AJ, Veeger D, van der Woude LH. Normative values and determinants of physical capacity in individuals with spinal cord injury. J Rehabil Res Dev 2002;39:29-39.
29. Haisma JA, Bussmann JB, Stam HJ, et al. Changes in physical capacity during and after inpatient rehabilitation in subjects with a spinal cord injury. Arch Phys Med Rehabil 2006;87:741-8.

30. Zoeller RF, Riechman SE, Dabayebeh IM, Goss FL, Robertson RJ, Jacobs PL. Relation between muscular strength and cardiorespiratory fitness in people with thoracic-level paraplegia. Arch Phys Med Rehabil 2005;86:1441-6.

31. Sniehotta FF, Scholz U, Schwarzer R. Action plans and coping plans for physical exercise: a longitudinal intervention study in cardiac rehabilitation. Br J Health Psychol 2010;11:23-37.

www.archives-pmr.org 
Chapter 3

\title{
Direct Referral and Physical Activity Counselling upon Discharge from Spinal Cord Injury Rehabilitation
}

\author{
Authors: Chelsea A. Pelletier, Amy E. Latimer-Cheung, Darren E. Warburton,
} Audrey L. Hicks 
Study Design: prospective cohort

Objectives: To evaluate the efficacy of direct referral from a health care provider to regular leisure time physical activity combined with 16-weeks of counselling support following discharge from either inpatient or outpatient spinal cord injury (SCI) rehabilitation.

Setting: Two rehabilitation centers in Canada

Methods: Seventeen participants (age $42.1 \pm 10.6$ years, C3 - T12, AIS A-C) were recruited at discharge from inpatient ( $\mathrm{n}=9 ; 5.2 \pm 1.3$ months post injury) or outpatient $(\mathrm{n}=8 ; 14.2 \pm 3.8$ months post injury) rehabilitation and were given a referral for regular exercise in accordance with the physical activity guidelines for adults with SCI.

Telephone counselling support was provided and adherence to the prescribed program was monitored for 16-weeks.

Results: There was a non-significant difference in exercise adherence rates (percentage of available sessions attended) between participants discharged from inpatient (71.4 \pm $44.9 \%)$ and those from outpatient $(39.5 \pm 27.2 \%)$ rehabilitation programs. The most common barriers to participation were physical health problems (50\%) and transportation (36\%). An exercise beliefs questionnaire revealed that participants value exercise at discharge from rehabilitation, despite the common report of a relative lack of confidence in their ability to perform or adapt aerobic and resistance exercises.

Conclusions: A system of direct referral and ongoing counselling support following discharge from either inpatient or outpatient SCI rehabilitation appears to encourage sustained participation, although future health promotion strategies should continue to 
PhD Thesis - Chelsea A. Pelletier - McMaster University - Kinesiology

provide specific information about how to adapt and complete aerobic and resistance exercises.

Sponsorship: Rick Hansen Institute and Ontario Neurotrauma Foundation

Keywords: Exercise, disability, health promotion, community, rehabilitation, spinal cord injury 


\section{Introduction}

The spinal cord injury (SCI) population remains largely inactive, placing individuals at increased risk for lifestyle related chronic illness. While several systematic reviews and the development of physical activity guidelines specific to the SCI population identify many fitness, and presumably health, benefits to regular physical activity participation, there is a significant need for improved health promotion strategies. ${ }^{1,2}$

One of the largest gaps in literature surrounds the acute and sub-acute phase of injury, specifically the point of discharge from rehabilitation. Leisure time physical activity (LTPA) rates decrease with increasing time post-injury, ${ }^{3}$ and longitudinal assessments have revealed a significant drop in activity status and an increase in body mass index in the months immediately following discharge from rehabilitation. ${ }^{4,5}$ From a theoretical perspective, many proven interventions incorporating plans to overcome barriers and translate intentions into behaviour would be ideal during this transitional period as individuals value exercise and can identify many benefits to regular participation; however, there also appears to be a high prevalence of barriers. ${ }^{6-9}$ A counselling program beginning during rehabilitation and continuing post-discharge has proven to be effective at increasing sport and physical activity participation at 9-weeks and 1-year postdischarge.$^{10,11}$ However, this intervention included participants with any neurological disorder, and was not specific to SCI. 
In the chronic SCI population, social cognitive theory has been shown to be predictive of future physical activity participation. ${ }^{12}$ This theory of behaviour change incorporates aspects of perceived control over one's behaviour, and includes outcome expectations and self-efficacy as important constructs. ${ }^{13}$ Self-efficacy specifically has been linked to future aerobic and resistance physical activity participation, ${ }^{14}$ although these relationships have not been demonstrated in adults with SCIs less than one year in duration.

With a current trend towards decreasing length of rehabilitation stays, the health care system is becoming increasingly reliant on outpatient services for long-term maintenance of health and function after SCI. ${ }^{15,16}$ A recent evaluation of current rehabilitation programs in Canada revealed an absence of structured exercise programs and exercise professionals, indicating few opportunities to support exercise engagement postdischarge.${ }^{15}$ Less than half of people with SCI report receiving any information about physical activity from their health care provider, and even fewer report receiving specific information about how and when to exercise. ${ }^{17,18}$ This fact is of particular importance as traditional rehabilitation on its own does not appear to provide enough cardiovascular strain to improve fitness. ${ }^{19}$ Further, physicians are consistently recognized as an important source of LTPA information, ${ }^{20}$ and specific preparation for LTPA during rehabilitation can serve as an important facilitator for continued participation. ${ }^{8}$ In the cardiac rehabilitation model, the strength of physicians referral is the most significant predictor of long-term adherence, ${ }^{21}$ and literature reviews suggest that patients are more likely to adhere to programs when they are referred, educated, and have a high amount of self- 
efficacy. ${ }^{22}$ Thus, from a health promotion perspective, this transitional period may prove to be highly influential as individuals reintegrate into the community and form new habits while still maintaining regular contact with health care providers.

The purpose of this study was to $i$ ) evaluate and compare the effectiveness of a direct referral to structured exercise and continued physical activity counselling upon discharge from either outpatient or inpatient SCI rehabilitation programs and ii) to evaluate the relationships between constructs of social cognitive theory and exercise adherence postdischarge. It was predicted that this system of direct referral and counselling would result in an increase in exercise participation among the study participants throughout the 16week follow-up period, and that the constructs of social cognitive theory (specifically self-efficacy) would have a strong correlation with LTPA participation. Further, it was predicted that adherence to the prescribed program and exercise self-efficacy would be greater among participants discharged from outpatient rehabilitation.

\section{Methods}

\section{Participants}

Participants were recruited at discharge from either inpatient or outpatient programs at SCI rehabilitation centres in Hamilton, Ontario and Vancouver, British Columbia, Canada from July 2011-February 2013. Participants were classified into one of two groups based 
on discharge program (inpatient or outpatient). Individuals who were 18-65 years old, wheelchair dependent, and could understand English were invited to participate. Written, informed consent was obtained from each participant, and the relevant research ethics board at each study site approved the protocol.

Physical Activity Referral and Counselling

Participants received a physical activity referral from their physiotherapist prior to discharge from either SCI rehabilitation program. The referral advised regular exercise in accordance with the physical activity guidelines for adults with $\mathrm{SCI}^{2}$ and could be completed as unstructured LTPA or as part of a structured community exercise program. Injury-specific demographic information, physical limitations, and any relevant rehabilitation outcomes were also included and provided to the program coordinator, if applicable.

In addition, participants received continuous physical activity counselling and support for a 16-week follow-up period after discharge. Trained research staff familiar with exercise in the SCI population completed the counselling protocol. The counselling was based on theoretical interventions previously used in this population and developed to be specific for participants at this stage of rehabilitation. ${ }^{6,7}$ Specifically, the protocol involved reviewing the benefits of LTPA, setting goals, and creating plans to both achieve goals (action planning) and overcome potential barriers (coping planning). After the initial 
telephone counselling session (lasting approximately 1 hour), follow-up phone calls were conducted every 4-weeks to track LTPA, monitor and re-assess goals, and record salient barriers. If participants were not interested or willing to complete the counselling portion of the study, they were still included in the referral portion of the study and adherence was monitored.

\section{Adherence}

Adherence to the twice-weekly exercise prescription was monitored for a 16-week follow-up period. Adherence was monitored as attendance at a structured community exercise program or as self-report for those who chose to exercise at home. For selfreport, sessions were only counted if they fit within the exercise referral (i.e., at least 20minutes of aerobic exercise or 3 sets of 10 repetitions of resistance exercise). Adherence rate was calculated based on a percentage of a maximum of 32 exercise sessions ( 2 times/week for 16-weeks). Reasons for not completing the exercise program were recorded as barriers, and prevalence was defined as the number of participants reporting each barrier at least once during the follow-up period.

\section{Exercise Beliefs Questionnaire}

Perceptions about exercise post-discharge were assessed with a questionnaire administered at the point of discharge, before the initial counselling session. Participants 
were asked to provide their opinions about engaging in regular LTPA over the next 16weeks. LTPA was defined as any activity that individuals choose to do in their free time that require exertion. Specifically, the questionnaire was framed around the constructs of social cognitive theory and assessed outcome expectations, scheduling self-efficacy, and task self-efficacy. ${ }^{13}$ All questions were rated on a seven-point scale $(1=$ not at all confident or strongly disagree and $7=$ completely confident or strongly agree). Mean composite scores were calculated for each construct for each participant.

Task self-efficacy was measured for four different types of exercise (moderate and heavy intensity aerobic and strengthening activity) and participants were asked to rate their confidence to engage in each activity for a) $10 \mathrm{~min}$, b) $20 \mathrm{~min}$, c) $30 \mathrm{~min}$, d) $45 \mathrm{~min}$, and e) 60 min without stopping. Internal consistency was good for both measures $\left(\alpha_{\text {aerobic }}=\right.$ $\left.0.85, \alpha_{\text {strengthening }}=0.93\right) .{ }^{23}$ Scheduling self-efficacy was measured by asking participants to rate their confidence in their ability to include 30 minutes of LTPA into their schedule a) one, b) two, c) three, and d) more than three times per week $(\alpha=0.67)$.

Outcome value had acceptable internal consistency $(\alpha=0.77)^{23}$ and was measured with three items: "in the next three months post discharge, engaging in regular LTPA is the most important thing I can do," "establishing a routine including regular LTPA in the next three months post discharge is a high priority for me" and "engaging in regular leisure time physical activity post discharge would be extremely valuable." Outcome expectations were measured with twelve items that were preceded with the statement " I 
think that engaging in regular leisure time physical activity in the next three months postdischarge will..." and participants were asked to rate various health and lifestyle outcomes specific to SCI. Internal consistency was adequate $(\alpha=0.71){ }^{23}$

Data Analysis

All data are presented as mean $\pm \mathrm{SD}$. Group differences in demographic information and self-efficacy constructs were compared with an independent t-test. Adherence rates between groups were compared using a one-way analysis of variance. Pearson's correlation coefficients $(r)$ were calculated between each self-efficacy construct and adherence. Significance was accepted at $\mathrm{p}<0.05$.

\section{Results}

\section{Participants}

Seventeen participants were recruited to participate in this study and divided into groups based on discharge program: inpatient discharge $(n=9)$ and outpatient discharge $(n=8)$. Overall the mean age ( $42.13 \pm 10.6$ years), sex ( $76 \%$ male), and causes of injury: motor vehicle accidents (44\%), falls (25\%), non-traumatic causes (19\%), and sport or recreation accidents (13\%) are representative of the SCI population..$^{24}$ The only significant group 
difference was in time post-injury $(\mathrm{p}=0.01)$, reflecting the two discharge time points. Participant characteristics are summarized in table 1.

\section{Adherence}

Two participants were lost to follow-up and are not included in adherence calculations; adherence for the remaining 15 participants is presented in figure 1. Overall, participants attended an average of $17.4 \pm 12.4$ sessions (out of a possible 32 ), corresponding to an adherence rate of $54.4 \pm 38.8 \%$. For participants discharged from inpatient rehabilitation, the mean number of sessions attended was $22.9 \pm 14.4$, equating to $71.4 \pm 44.9 \%$. Participants discharged from an outpatient rehabilitation setting completed a mean of 12.6 \pm 8.7 sessions or $39.5 \pm 27.2 \%$. Among the participants discharged from outpatient rehabilitation, 4 were not interested in the follow-up counselling, but were still given the referral. The values were different for these two groups (referral plus counselling: $50.0 \pm$ 18.2\%; referral only: $28.9 \pm 33.2 \%$ ). None of these group differences in attendance rates were significant $(\mathrm{p}>0.05)$. Prevalence of common barriers is presented in table 2 .

\section{Exercise Beliefs Questionnaire}

Results from the exercise beliefs questionnaire and correlations between the constructs of social cognitive theory and adherence are presented in table 3 . There were no significant 
differences $(p>0.05)$ in any of the constructs measured between groups. There were also no significant correlations between any of the constructs and adherence rates.

\section{Discussion}

The purpose of this study was to evaluate the effectiveness of direct referral to regular exercise upon discharge from inpatient or outpatient SCI rehabilitation. In addition, we aimed to explore if a continued health promotion strategy involving telephone counselling support would be beneficial to long-term LTPA participation post-discharge. Findings of this pilot project indicate the potential role of such an initiative in the rehabilitation process, as promising adherence rates support the efficacy of this model of care.

The exercise adherence rates of $71 \%$ and $50 \%$ among those who completed the counselling protocol are a favourable finding, and 6 of the 11 participants who finished the protocol had adherence rates above $75 \%$, with 4 reporting rates of $100 \%$. While comparisons to other reported adherence rates are difficult, these rates do appear to be higher than LTPA rates typically reported in this population and seemingly counteract the decrease in participation that has been reported during this time period. ${ }^{4,17,18}$ It is also encouraging to note the difference in adherence rates between participants who received the counselling and those that did not (64\% vs. $28.9 \%)$. While the sample for this portion of the study is small and the high amount of individual variability likely contributes to the lack of statistical significance, it offers putative support for the importance of the 
counselling component during the critical first months after discharge from rehabilitation. The adherence rates reported in the current study are similar to those that have been reported in a cardiac rehabilitation model, in which a combined action and coping planning intervention produced a $71 \%$ adherence rate to the prescribed exercise program, versus just $42 \%$ in a control condition. ${ }^{25}$

The most common barriers to exercise participation in the present study were physical health problems (i.e., urinary tract infections and general illness), transportation, and personal life situations (i.e., moving and return to work). These findings are consistent with those that have been reported previously with respect to regular physical activity among individuals who are newly discharged. ${ }^{8}$ While several studies in the chronic SCI population indicate internal barriers (i.e., motivation) as a strong predictor of behaviour, ${ }^{17,18}$ this was not reported in the present study. Although we did not measure motivation or changes in the constructs of social cognitive theory as a result of our counselling intervention, the counselling support may have helped participants maintain interest in exercise. Similarly, resource barriers (i.e., accessibility of facilities and knowledgeable staff) is one of the most commonly reported barriers for exercise participation $^{8,17,26}$ and community reintegration after SCI, ${ }^{27}$ and was not reported by the participants in the present study. The differences in reported barriers may be due to a combination of factors including the counselling intervention but also community exercise programs available near the study sites. 
Another interesting finding is the similarity between self-efficacy scores between people being discharged from inpatient and those from outpatient rehabilitation programs. These values are similar to what we have recently reported in the sub-acute SCI population during inpatient rehabilitation; ${ }^{9}$ that these self-efficacy values were not higher following an outpatient rehabilitation program is perhaps reflective of the lack of specific community-based exercise education and opportunities available to this population. It is also interesting to note the lack of relationship between the self-efficacy constructs and LTPA participation, as social cognitive theory has been shown to be a predictor of exercise behaviour in previous studies in the chronic SCI population. ${ }^{12,14}$ Task and scheduling self-efficacy have also been associated with adherence to maintenance cardiac rehabilitation programs. ${ }^{28}$ The reasons for this discrepancy may be due to the relatively low levels of task self-efficacy reported in the current study and the high amount of variability reported in adherence rates. One limitation of this work is that we did not readminister the questionnaire following the counselling protocol or at the end of the intervention. Further investigation may reveal stronger associations between the constructs of social cognitive theory and exercise behaviour post-discharge.

Recently, Rimmer ${ }^{29}$ proposed a model describing the short-term gains in health and function achieved during rehabilitation, which then plateau following discharge. During this transitional period, individuals reach a point at which their health trajectory can either increase or decrease depending on the initiation of an effective exercise program. Further a transitional therapist-to-trainer model has been proposed, similar to the intervention 
used in the current study, wherein collaboration is fostered between health care providers (i.e., physiotherapists) and qualified exercise specialists to develop an exercise prescription that addresses specific physiological, environmental, and motivational challenges that individuals face post-discharge from rehabilitation. ${ }^{30}$ While this model remains theoretical, this study has been the first to examine a means by which to translate it into practice, and future studies should be encouraged to expand on this system of care.

\section{Conclusions}

The results of this study support the effectiveness of adding an exercise referral from a health care provider and counselling support from specialized staff to the rehabilitation model after SCI. The linking of rehabilitation to community exercise may be an important model for health promotion and education in other areas of self-care as individuals reintegrate into the community following disability. 


\section{References}

1. Hicks AL, Martin Ginis KA, Pelletier CA, Ditor DS, Foulon B, Wolfe DL. The effects of exercise training on physical capacity, strength, body composition and functional performance among adults with spinal cord injury: a systematic review. Spinal Cord 2011;49:1103-27.

2. Ginis KAM, Hicks AL, Latimer AE, Warburton DER, Bourne C, Ditor DS, et al. The development of evidence-informed physical activity guidelines for adults with spinal cord injury. Spinal Cord 2011;49:1088-96.

3. Ginis KAM, Latimer AE, Arbour-Nicitopoulos KP, Buchholz AC, Bray SR, Craven $\mathrm{BC}$, et al. Leisure time physical activity in a population-based sample of people with spinal cord injury part I: demographic and injury-related correlates. Arch Phys Med Rehabil 2010;91:722-8.

4. van den Berg-Emons RJ, Bussmann JB, Haisma JA, Sluis TA, van der Woude LH, Bergen MP, et al. A prospective study on physical activity levels after spinal cord injury during inpatient rehabilitation and the year after discharge. Arch Phys Med Rehabil 2008;89:2094-101.

5. de Groot S, Post M, Postma K, Sluis TA, van der Woude L. Prospective analysis of body mass index during and up to 5 years after discharge from inpatient spinal cord injury rehabilitation. J Rehabil Med 2010;42:922-8.

6. Latimer AE, Ginis KAM, Arbour KP. The efficacy of an implementation intention intervention for promoting physical activity among individuals with spinal cord injury: A randomized controlled trial. Rehabil Psychol 2006;51:273-80.

7. Arbour-Nicitopoulos KP, Ginis KAM, Latimer AE. Planning, leisure-time physical activity, and coping self-efficacy in persons with spinal cord injury: a randomized controlled trial. Arch Phys Med Rehabil 2009;90:2003-11.

8. Vissers M, van den Berg-Emons R, Sluis T, Bergen M, Stam H, Bussmann H. Barriers to and facilitators of everyday physical activity in persons with a spinal cord injury after discharge from the rehabilitation centre. J Rehabil Med 2008;40:461-7.

9. Pelletier CA, Jones G, E Latimer-Cheung A, Warburton DE, Hicks AL. Aerobic capacity, orthostatic tolerance, and exercise perceptions at discharge from inpatient spinal cord injury rehabilitation Arch Phys Med Rehabil 2013, doi:10.1016/j.apmr.2013.05.011 
10. Van der Ploeg HP, Streppel K, Van der Beek AJ, van der Woude L, VollenbroekHutten M, Van Harten WH, et al. Counselling increases physical activity behaviour nine weeks after rehabilitation. Brit J Sports Med 2006;40:223-9.

11. van der Ploeg HP, Streppel KRM, van der Beek AJ, van der Woude LHV, Vollenbroek-Hutten MMR, van Harten WH, et al. Successfully improving physical activity behavior after rehabilitation. Am J Health Promot 2007;21:153-9.

12. Ginis KAM, Latimer AE, Arbour-Nicitopoulos KP, Bassett RL, Wolfe DL, Hanna SE. Determinants of physical activity among people with spinal cord injury: a test of social cognitive theory. Ann Behav Med 2011;42:127-33.

13. Bandura A. Health promotion by social cognitive means. Health Educ Behav 2004;31:143-64.

14. Kroll T, Kratz A, Kehn M, Jensen MP, Groah S, Ljungberg IH, et al. Perceived exercise self-efficacy as a predictor of exercise behavior in individuals aging with spinal cord injury. Am J Phys Med Rehabil 2012;91:640-51.

15. Craven C, Verrier M, Balioussis C, Wolfe DL, J H, Noonan V, et al. Capturing capacity in Canadian SCI rehabilitation. Rick Hansen Institute; 2012.

16. Whiteneck GG, Gassaway J, Dijkers MP, Lammertse DP, Hammond F, Heinemann AW, et al. Inpatient and postdischarge rehabilitation services provided in the first year after spinal cord injury: findings from the SCIRehab study. Arch Phys Med Rehabil 2011;92:361-8.

17. Scelza WM, Kalpakjian CZ, Zemper ED, Tate DG. Perceived barriers to exercise in people with spinal cord injury. Am J Phys Med Rehabil 2005;84:576-83.

18. Cowan RE, Nash MS, Anderson KD. Exercise participation barrier prevalence and association with exercise participation status in individuals with spinal cord injury. Spinal Cord 2013;51:27-32.

19. Koopman ADM, Eken MM, van Bezeij T, Valent LJM, Houdijk H. Does clinical rehabilitation impose sufficient cardiorespiratory strain to improve aerobic fitness? J Rehabil Med 2012;

20. Glasgow RE, Eakin EG, Fisher EB, Bacak SJ, Brownson RC. Physician advice and support for physical activity: results from a national survey. Am J Prev Med 2001;21:189-96.

21. Ades PA, Waldmann ML, McCann WJ, Weaver SO. Predictors of cardiac rehabilitation participation in older coronary patients. Arch Intern Med 1992;152:1033-5. 
PhD Thesis - Chelsea A. Pelletier - McMaster University - Kinesiology

22. Jackson L. Getting the most out of cardiac rehabilitation: a review of referral and adherence predictors. Heart 2005;91:10-4.

23. Nunnally JC. Psychometric theory. 2nd ed. New York: McGraw-Hill; 1978.

24. Pickett GE, Campos-Benitez M, Keller JL, Duggal N. Epidemiology of traumatic spinal cord injury in Canada. Spine 2006;31:799-805.

25. Sniehotta FF, Scholz U, Schwarzer R. Action plans and coping plans for physical exercise: A longitudinal intervention study in cardiac rehabilitation. Brit J Health Psychol 2010;11:23-37.

26. Rimmer JH, Riley B, Wang E, Rauworth A, Jurkowski J. Physical activity participation among persons with disabilities: barriers and facilitators. Am J Prev Med 2004;26:419-25.

27. Scelza WM, Kirshblum SC, Wuermser L-A, Ho CH, Priebe MM, Chiodo AE. Spinal cord injury medicine. 4. Community reintegration after spinal cord injury. Arch Phys Med Rehabil 2007;88:S71-5.

28. Woodgate J, Brawley LR, Weston ZJ. Maintenance cardiac rehabilitation exercise adherence: effects of task and self-regulatory self-efficacy. J Appl Social Pyschol 2005;35:183-222.

29. Rimmer JH. Getting beyond the plateau: bridging the gap between rehabilitation and community-based exercise. PMRJ 2012;4:857-61.

30. Rimmer JH, Henley KY. Building the crossroad between inpatient/outpatient rehabilitation and lifelong community-based fitness for people with neurologic disability. J Neurol Phys Ther 2013;37:72-7. 
PhD Thesis - Chelsea A. Pelletier - McMaster University - Kinesiology

Table 1. Participant characteristics

\begin{tabular}{lccc}
\hline Variable & Overall & $\begin{array}{c}\text { Inpatient } \\
\text { Discharge }\end{array}$ & $\begin{array}{c}\text { Outpatient } \\
\text { Discharge }\end{array}$ \\
\hline Sample size $(n)$ & 17 & 9 & 8 \\
Age (years) & $42.1 \pm 10.6$ & $38.6 \pm 10.6$ & $46.7 \pm 9.4$ \\
Sex (males/females) & $13 / 4$ & $7 / 2$ & $6 / 2$ \\
Level of Injury & $\mathrm{C} 3-\mathrm{T} 12$ & $\mathrm{C} 4-\mathrm{T} 12$ & $\mathrm{C} 3-\mathrm{T} 12$ \\
AIS & $\mathrm{A}-\mathrm{C}$ & $\mathrm{A}-\mathrm{C}$ & $\mathrm{B}-\mathrm{C}$ \\
Time Post Injury (months) & $8.43 \pm 5.1$ & $5.2 \pm 1.3$ & $14.2 \pm 3.8^{*}$ \\
\hline
\end{tabular}

Values are mean \pm SD. * indicates a significant difference between groups $(\mathrm{p}<0.05)$

Abbreviations: AIS, ASIA impairment score 
PhD Thesis - Chelsea A. Pelletier - McMaster University - Kinesiology

Table 2. Prevalence of barriers to physical activity participation

\begin{tabular}{ll}
\hline Barrier & Prevalence (number of participants) \\
\hline Physical Health Problems & $7(50 \%)$ \\
Transportation & $5(36 \%)$ \\
Personal Life Situation & $4(29 \%)$ \\
Mental Health Issues & $1(7 \%)$ \\
\hline
\end{tabular}


PhD Thesis - Chelsea A. Pelletier - McMaster University - Kinesiology

Table 3. Mean composite scores for outcome value, outcome expectation, scheduling, and task self-efficacy.

\begin{tabular}{lccc}
\hline Scale & $\begin{array}{c}\text { Inpatient } \\
\text { Discharge }\end{array}$ & $\begin{array}{c}\text { Outpatient } \\
\text { Discharge }\end{array}$ & $\begin{array}{c}\text { Correlation with } \\
\text { Adherence }\end{array}$ \\
\hline Outcome Value & $6.3 \pm 0.75$ & $6.1 \pm 0.75$ & $r=0.03, p=0.94$ \\
Outcome Expectations & $6.0 \pm 0.70$ & $6.0 \pm 0.50$ & $r=-0.16, p=0.64$ \\
Scheduling SE & $6.0 \pm 0.90$ & $5.9 \pm 1.0$ & $r=0.46, p=0.10$ \\
Task SE: Aerobic Exercise & $4.0 \pm 1.0$ & $4.7 \pm 1.3$ & $r=0.49, p=0.07$ \\
Task SE: Strengthening Exercise & $4.5 \pm 1.3$ & $4.5 \pm 1.4$ & $r=0.24, p=0.42$ \\
\hline
\end{tabular}

Values are mean \pm SD. Abbreviations: SE, self-efficacy. 

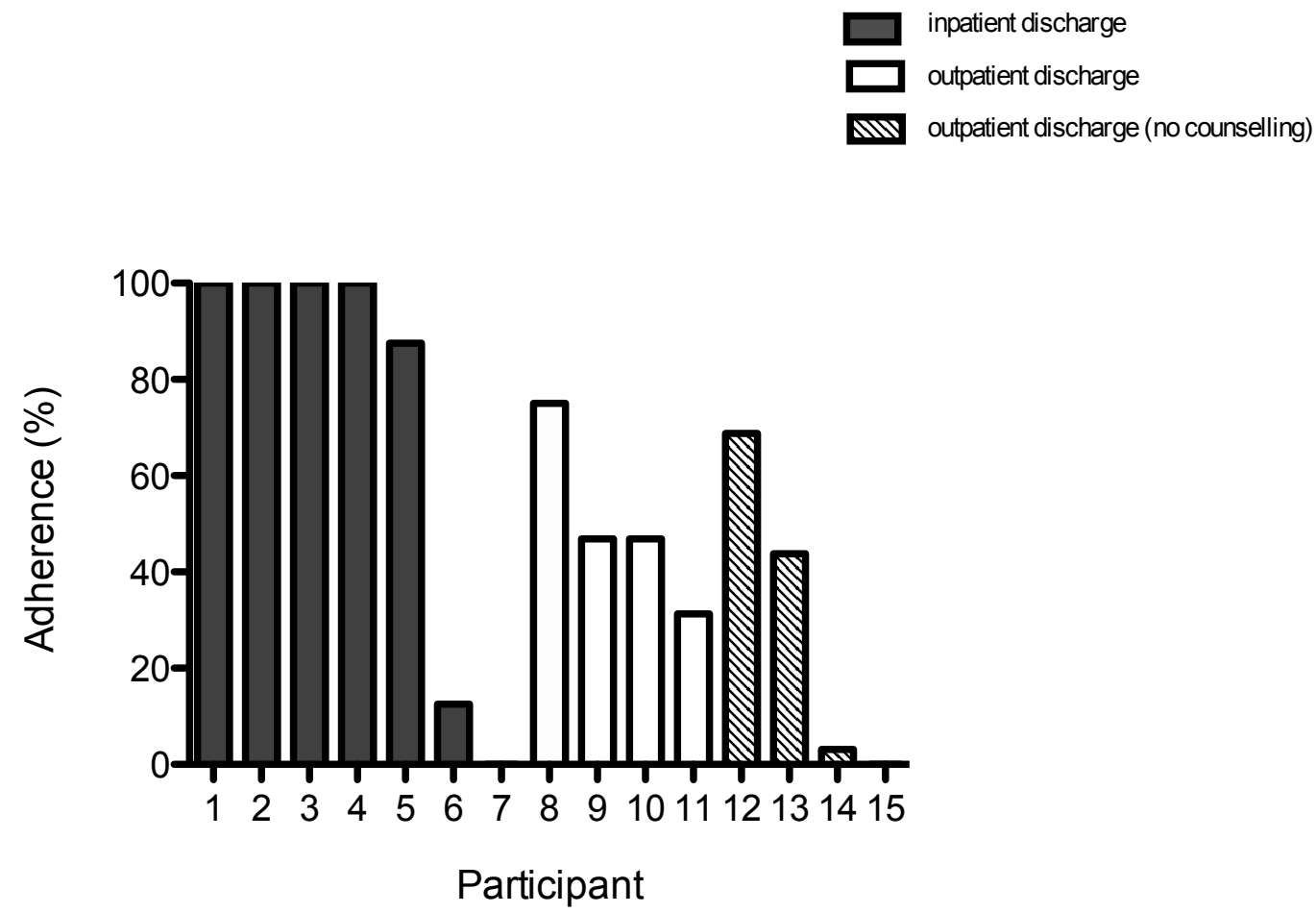

Figure 1. Adherence rate (\% of prescribed sessions) for each participant during a 16week follow-up period 
Chapter 4

\section{The Optimal Modes of Exercise for Individuals with Spinal Cord Injury: Consumer Preference and Metabolic Demand}

Authors: Chelsea A. Pelletier, David S. Ditor, Amy E. Latimer-Cheung, Darren E. Warburton, Audrey L. Hicks 
Study Design: cross-sectional

Objectives: To compare four types of aerobic exercise equipment: arm cycle ergometer (ACE), arm glider (AG), arm + leg recumbent stepper (ALRS), and arm + leg cycle ergometer (ALCE) and two pieces of resistance exercise equipment: wall pulleys (WP) and weight stack (WS) in adults with tetraplegia (TP; C3-C8) and paraplegia (PP; T3T12).

Setting: Community exercise programs in Ontario and British Columbia, Canada Methods: Thirty-six participants (age: $41.1 \pm 12.1$ years, $11.4 \pm 10.7$ years post-injury) completed 20 minutes of submaximal aerobic exercise or 3 sets of 10 repetitions of resistance exercise on each type of equipment. Heart rate (HR), oxygen uptake $\left(\mathrm{VO}_{2}\right)$, and 10-point ratings of perceived exertion (RPE) were measured during aerobic exercise. A questionnaire was administered to evaluate consumer opinions.

Results: There were significant group but not equipment differences in HR (TP: 101.4 bpm; PP: $124.9 \mathrm{bpm}$ ) and $\mathrm{VO}_{2}$ (TP: $6.5 \mathrm{~mL} \cdot \mathrm{kg}^{-1} \cdot \mathrm{min}^{-1}$; PP: $10.5 \mathrm{~mL} \cdot \mathrm{kg}^{-1} \cdot \mathrm{min}^{-1}$ ) during submaximal exercise. There was a significant increase in RPE among participants with TP during both ACE and ALRS exercise. While all participants were willing to use ACE, only $65 \%$ of TP and $68 \%$ of PP groups were willing to use ALRS due to difficulties with transferring. ACE, AG, ALRS, WP, and WS were perceived as more safe by participants with PP than by those with TP.

Conclusions: There appears to be no metabolic or cardiovascular advantage to passive hybrid exercise versus arm-only exercise in participants with TP or PP. Health promotion 
PhD Thesis - Chelsea A. Pelletier - McMaster University - Kinesiology

strategies should promote arm-only exercise modes as they appear to be better received by a broader range of individuals with spinal cord injury.

Sponsorship: Rick Hansen Institute and Ontario Neurotrauma Foundation

Keywords: tetraplegia, paraplegia, energy expenditure, exercise prescription 


\section{Introduction}

The evidence-informed physical activity guidelines for adults with spinal cord injuries (SCI) recommends twice-weekly exercise consisting of 20-minutes of moderate to vigorous aerobic exercise and 3 sets of 10-12 repetitions of strengthening exercise of each major muscle group. ${ }^{1,2}$ There is currently limited information regarding which mode of exercise is ideal for achieving this prescription or which types of exercise the consumer prefers. Further, for individuals with SCI above T6, the abnormal heart rate (HR) response to exercise makes it difficult to prescribe or predict exercise intensity.

A significant health concern following SCI is a negative change in body composition marked by a decrease in fat free mass and an increase in fat mass, placing individuals at increased risk for cardiovascular and metabolic diseases. ${ }^{3-5}$ These changes, combined with a decrease in physical activity, contribute to a $14-27 \%$ decrease in resting metabolic rate which further perpetuates a positive energy balance. ${ }^{3,6,7}$ The metabolic cost of exercise relative to rest is also presumably lower after SCI; the value of one metabolic equivalent (1 MET) has been estimated at $2.7 \mathrm{~mL} \cdot \mathrm{kg}^{-1} \cdot \mathrm{min}^{-1}$ compared to the traditional $3.5 \mathrm{~mL} \cdot \mathrm{kg}^{-}$ ${ }^{1} \cdot \min ^{-1}$ used in the able-bodied literature. ${ }^{8}$

There are few studies that have attempted to compare metabolic demand between adapted exercise modes in the SCI population. The majority have used functional electrical stimulated (FES) exercise and have reported greater oxygen requirements for hybrid FES 
rowing compared to arms only rowing ${ }^{9}$ and between FES hybrid cycling and arm cycling. ${ }^{10}$ Further, a study by Hasnan et al. ${ }^{11}$ revealed that both arm exercise and hybrid FES arm and leg exercise elicited a higher oxygen demand and HR response compared with FES leg exercise alone. Accordingly, electrically stimulating paralyzed muscle may provide a certain amount of metabolic advantage during exercise, although the equipment is expensive and not typically available in community exercise facilities.

Due to the autonomic dysfunction and reliance on arm exercise following SCI, traditional relationships between $\mathrm{HR}$ and oxygen consumption $\left(\mathrm{VO}_{2}\right)$ cannot be assumed. Thus, common indices used for prescribing exercise intensity, such as a percentage of maximum HR, cannot be applied for this population. Several studies have explored alternative means to prescribe exercise intensity, most commonly Borg's ratings of perceived exertion (RPE). ${ }^{12}$ While some previous studies using arm cycle ergometry have reported a poor relationship between RPE and measures of $\mathrm{HR}$ or $\mathrm{VO}_{2},{ }^{13,14}$ others have demonstrated a strong correlation between these variables..$^{15} \mathrm{RPE}$ has also been shown to be effective in regulating exercise at a moderate or vigorous intensity. ${ }^{16}$

One important component of advancing the exercise prescription (and ensuring compliance) is identifying which type of equipment the consumer (individuals with SCI) prefers. It is critical from health promotion perspectives to identify which pieces of equipment are preferred and should thus be purchased (and encouraged) by community service providers. There is very limited information available on consumer preference of 
exercise equipment; one study has shown that people with SCI prefer exercise equipment with the lowest energy cost,${ }^{17}$ although this study was limited and involved a comparison of only two pieces of exercise equipment.

The purpose of this study was to compare four types of commercially available aerobic exercise equipment and two types of resistance exercise equipment in terms of cardiovascular response, metabolic demand, and consumer preference based on level of injury. It was predicted that hybrid arm and leg aerobic exercise would be both preferred by the consumer and would elicit a higher cardiovascular and metabolic response and that there would be no difference in preference between wall pulley and traditional weight stack resistance exercise modes. It was also hypothesized that individuals with tetraplegia would have a lower $\mathrm{VO}_{2}$ and $\mathrm{HR}$ response to submaximal exercise compared to those with paraplegia, and there would be no difference in any measure of consumer preference based on level of injury.

\section{Methods}

\section{Participants}

Participants were recruited from four community exercise programs for adults with SCI in Ontario and British Columbia, Canada. Inclusion criteria were adults with a chronic SCI (> 1 year post injury) who were 18-65 years old, wheelchair dependent, and able to 
understand English. Participants were classified according to level of injury: tetraplegia (TP; C1-C8) and paraplegia (PP; T1-T12). Written, informed consent was obtained from each participant, and ethics approval was obtained from the relevant Research Ethics Board at each study site.

\section{Submaximal Aerobic Exercise}

Participants were asked to complete 20-minutes of submaximal aerobic exercise at a moderate to vigorous intensity (3-6 on the 10-point RPE) to simulate the recommended exercise prescription for this population. ${ }^{1} \mathrm{HR}$ was continually monitored throughout the 30-minute protocol (5-minutes of rest, 20-minutes of exercise, 5-minutes of recovery) with a chest monitor (Polar Electro Canada, Lachine, Quebec, Canada), and RPEs were recorded every 5 minutes. ${ }^{12}$ Each testing session was completed on separate nonsequential days, and the order of testing was randomized. Tensor bandages were used to secure the hands of participants with insufficient grip strength. Four categories of aerobic exercise equipment were compared: arm cycle ergometer (ACE; Monark Arm Ergometer Rehab Trainer, Patterson Medical Supply Inc., Mississauga, Ontario, Canada); arm glider (AG; Vitaglide Pro Glider, RMT Fitness, Miami, Florida, United States); hybrid arm and leg recumbent stepper (ALRS; NuStep T5XR Recumbent Cross Trainer, NuStep Inc., Ann Arbor, Michigan, United States; SCI Fit 7000 Total Body Recumbent Stepper, SCI Fit Ltd., Tulsa, Oklahoma, United States); and hybrid arm and leg cycle ergometer (ALCE; SCI Fit Pro 2 Total Body with Adjustable Cranks, SCI Fit Ltd., Tulsa, 
Oklahoma, United States). Each study site had a different combination of exercise equipment available; however, all study sites followed a mutually agreed upon protocol, and sessions were completed by trained staff familiar with the equipment and exercise training in this population. Participants were allowed to choose which pieces of equipment they were comfortable using and were not required to use all four pieces of aerobic exercise equipment in order to participate.

\section{Metabolic Demand}

In a subset of participants $(\mathrm{n}=9)$ at one study site, $\mathrm{VO}_{2}\left(\mathrm{VO}_{2000}\right.$ Metabolic Measurement System, MGC Diagnostics Corp., St. Paul, Minnesota, United States) was measured throughout the exercise protocol on each of three pieces of aerobic exercise equipment (ACE, AG, ALRS) to obtain an assessment of metabolic demand. Steady state HR and $\mathrm{VO}_{2}$ were defined as the mean value during 20-minutes of submaximal exercise. Prior to this assessment, participants completed a peak exercise test on an ACE (Lode B.V., Groningen, the Netherlands; Moxus Metabolic System, AEI Technologies, Inc., Bastrop, Texas, United States). This protocol has been used previously in this population ${ }^{18,19}$ and included an incremental ramp protocol where the resistance was increased by $5 \mathrm{~W} / \mathrm{min}$ for those with $\mathrm{TP}$ and $10 \mathrm{~W} / \mathrm{min}$ for those with $\mathrm{PP}$ until volitional fatigue. $\mathrm{VO}_{2 \text { peak }}$ and $\mathrm{HR}_{\text {peak }}$ were defined as the maximal value achieved in a 30-second interval. Peak power output $\left(\mathrm{PO}_{\text {peak }}\right)$ was defined as the maximal resistance achieved and maintained for at least 15 seconds. 
Resistance Exercise

Two pieces of resistance exercise equipment were evaluated, wall pulleys (WP; Endorphin Pulleys, Patterson Medical Canada, Mississauga, Ontario, Canada) and traditional weight stack equipment (WS; Equalizer Multi-station, Equalizer Exercise Machines, Red Deer, Alberta, Canada; Cybex VR3 Total Access, Fitness Town Commercial, Vancouver, British Columbia, Canada). Participants were asked to complete 3 sets of 10 repetitions of one exercise using each major muscle group (i.e., chest, back, biceps, triceps), depending on their own abilities. ${ }^{1}$ Wrist cuffs and clips were used for participants with insufficient grip strength.

\section{Consumer Preference Questionnaire}

Participants were asked to complete a questionnaire to assess their thoughts about each piece of exercise equipment immediately following use. Pain was measured using three scales modified from the brief pain inventory and were rated on a 7-point scale $(1=$ none at all, $7=\mathrm{a}$ lot $){ }^{20,21}$ Participants were asked to rate how much shoulder pain, bodily pain, and physical discomfort they typically experience and how much they experienced using the specific piece of exercise equipment. Internal consistency was good for both measures $\left(\alpha_{\text {typical }}=0.84, \alpha_{\text {specific }}=0.81\right) .{ }^{22}$ Perceived safety of using each piece of equipment was also assessed with two items by asking participants to rate how confident they were $(1=$ not at all confident, $7=$ completely confident) in their ability to use each piece of exercise 
equipment a) without assistance and b) safely without causing injury. Internal consistency was acceptable for these items $(\alpha=0.74) .{ }^{22}$ Perceived enjoyment of using each type of equipment was measured using one scale "How much did you like using this specific piece of exercise equipment?", and answers were rated from $1=$ not at all to $7=$ a lot.

Finally, the questionnaire included specific questions to assess whether participants felt they could use each piece of aerobic and resistance exercise equipment to meet the physical activity guidelines for adults with SCI. ${ }^{1}$ Both resistance and aerobic exercise equipment questionnaires included the item, "Assuming that you are very motivated and fit, how many times per week could you imagine yourself using this specific piece of exercise equipment?" Participants were asked to select a) 1, b) 2, c) 3, d) 4, or e) more than 4. For aerobic exercise, participants were asked to selected how many minutes they could imagine using each specific piece of equipment: a) less than $5 \mathrm{~min}$, b) $5 \mathrm{~min}$, c) 10 min, d) $15 \mathrm{~min}$, e) $20 \mathrm{~min}$, f) $25 \mathrm{~min}, \mathrm{~g}) 30 \mathrm{~min}$, or h) other. Resistance equipment was specifically evaluated by two items: “Assuming you are very motivated, how many sets could you imagine doing while using this specific piece of exercise equipment", with options of a) less than 1, b) 1, c) 2, d) 3, and e) more than 3, and “Assuming you are very motivated, in one exercise session, how many repetitions could you imagine doing in each set using this specific piece of exercise equipment?" with options of a) less than 5, b) 5-8, c) $8-10$, d) $10-12$, e) $12-15$, and f) more than 15 . 
Data Analysis

All data are presented as means \pm SD and were analysed using Microsoft excel and IBM SPSS (Version 20.0, SPSS Inc., Chicago, Illinois, United States). Due to participants choosing not use certain exercise modes and the high number of missing data sets, independent t-tests were used to compare results between groups (TP vs. PP) and between equipment types (ACE, AG, ALRS, ALCE) for the consumer preference questionnaire. Group differences (TP vs. PP) in peak exercise test results were also compared using an independent samples t-test. Steady state $\mathrm{HR}$ and $\mathrm{VO}_{2}$ were analyzed with a two-way (group x equipment) analysis of variance (ANOVA). RPE was analyzed using a two-way (group x time) repeated measures ANOVA, using the Greenhouse-Geisser correction where the assumption of sphericity was violated.

\section{Results}

\section{Participants}

Participant characteristics are presented in table 1. Thirty-six participants were recruited to participate in this study. Of these subjects, 9 were recruited to complete the assessment of metabolic demand (age $40.1 \pm 10.8$ years, 8 males and 1 female, C3-T10, $10.2 \pm 11.5$ years post-injury). The most common causes of injury were motor vehicle accidents 
(35\%), falls (23\%), and sports/recreation (15\%), percentages which are consistent with national averages..$^{23}$

\section{Cardiovascular and Metabolic Responses to Submaximal Aerobic Exercise}

Results of the peak exercise test revealed a significant difference in $\mathrm{PO}_{\text {peak }}(\mathrm{p}=0.02)$ and relative $\mathrm{VO}_{2 \text { peak }}(\mathrm{p}=0.03)$ between TP and PP groups (Table 2). Physiological responses to submaximal exercise bouts are presented in Table 3, Figure 1 (HR), and Figure 2 $\left(\mathrm{VO}_{2}\right)$. There was a significant main effect for group for steady state $\mathrm{HR}(\mathrm{p}<0.01)$, absolute $\mathrm{VO}_{2}(\mathrm{p}<0.01)$, relative $\mathrm{VO}_{2}(\mathrm{p}<0.01)$, and $\% \mathrm{HR}_{\text {peak }}(\mathrm{p}=0.03)$ across three equipment types (ACE, AG, ALRS), where TP achieved lower values than PP for all variables. There was a significant group $\mathrm{x}$ time interaction for RPE during ACE $(\mathrm{p}=$ $0.01)$, ALRS $(\mathrm{p}=0.02)$, and ALCE $(\mathrm{p}=0.04)$, with a greater increase seen in TP across all exercise modes. RPE data are presented in Table 4.

\section{Consumer Preference}

Results of the consumer preference questionnaire are presented in Table 5. All participants in both groups completed the exercise bout using ACE (100\%), while only some were willing to complete the exercise bout using either ALRS (TP: 65\%. PP: 68\%) or ALCE (TP: 29\%, PP: 58\%). The majority of participants had experience with ACE (TP: 88\%, PP: 100\%), while fewer had previously used AG (TP: 45\%, PP: 36\%), ALRS 
(TP: 64\%, PP: 54\%), or ALCE (TP: 0\%, PP: 14\%). There were group differences in typical $(p=0.04)$ and specific pain $(p=0.04)$ after using ACE. There were no group or equipment differences in perceived enjoyment. Perceived safety was significantly lower in the TP group when comparing ACE to ALRS $(p=0.04)$ and AG to ALRS $(p<0.01)$. Similar results were seen in the PP group when comparing ACE to ALRS $(p=0.02)$, ACE to ALCE (p < 0.01), AG to ALRS (p = 0.02), and AG to ALCE $(p<0.01)$.

In the resistance training equipment, while most participants were willing to use both WP (TP: 91\%, PP: 100\%) and WS (TP: 100\%, PP: 100\%), not as many were familiar with WP (TP: 70\%, PP: 75\%). As with the aerobic equipment, perceived safety was lower in TP than PP for both WP $(\mathrm{p}=0.01)$ and WS $(\mathrm{p}=0.02)$, and there were no differences in perceived enjoyment.

Mean scores indicate that participants feel confident in their ability to use the aerobic and resistance exercise equipment to meet or exceed the physical activity guidelines.

\section{Discussion}

The purpose of this study was to identify the ideal mode of exercise for individuals with SCI in terms of cardiovascular response, metabolic demand, and consumer preference based on level of injury. Contrary to our hypothesis, there were no differences in HR or $\mathrm{VO}_{2}$ responses to steady state submaximal exercise between modes, but there were 
differences when comparing individuals with TP and PP. Similarly, there were no group or equipment differences in overall enjoyment of the various types of aerobic or resistance exercise equipment. There were significant group and equipment differences in terms of perceived safety of using arm only (ACE and AG) and hybrid equipment (ALRS and ALCE).

The lack of differences in energy expenditure between hybrid and arm only exercise was unexpected. Given that some individuals with incomplete SCI may retain the capacity to voluntary activate some muscles in the lower limbs, we anticipated that there might be greater muscle activation with the arm-leg exercise equipment than with arm-only. While previous work has shown a greater metabolic demand for hybrid vs. arms- or legs-only exercise in this population, ${ }^{8-11}$ all of these studies have utilized FES to activate lower body muscles. Therefore, it appears that in order for there to be any significant metabolic advantage to hybrid exercise, the equipment must involve electrical stimulation of inactive muscle tissue regardless of residual muscle activation.

One other interesting finding was the increase in RPE seen among participants with TP during submaximal exercise using ACE, ALRS, and ALCE despite no increase in PO or $\mathrm{VO}_{2}$. Similar results have been noted by Cowan et al., ${ }^{14}$ who suggested that participants may be using RPE as an indicator of peripheral rather than central fatigue. There are inconsistent reports of the validity of RPE in prescribing exercise in the SCI population. Lewis et al ${ }^{13}$ reported limited significant correlations between $\mathrm{HR}$ and $\mathrm{VO}_{2}$ in either $\mathrm{TP}$ 
or PP during a peak graded exercise test, particularly at a moderate intensity of effort. Conversely, Goosey-Tolfrey et al. ${ }^{16}$ have validated the use of RPE to prescribe exercise intensity, demonstrating no difference between an imposed or self-selected intensity submaximal exercise trial at a moderate or vigorous intensity; however participants in this study were given instructions on rating fatigue using the RPE. In the present study, participants were instructed to exercise according to verbal anchors of "moderate" and "very hard" used on the RPE scale, yet the corresponding oxygen uptakes represented only $40-50 \%$ of $\mathrm{VO}_{2 \text { peak }}$ (TP: 40.8-46.2\%; PP: 44.4-50.8\%). These findings might suggest that participants will naturally select the 'easiest' intensity when presented with a range to achieve, and to attain the intensity of effort associated with the physical activity guidelines perhaps an RPE range of 4-7 would be more appropriate. ${ }^{12}$

It was encouraging to note that there were no significant differences in steady state $\mathrm{VO}_{2}$ or $\% \mathrm{VO}_{2 \text { peak }}$ between equipment modes, suggesting that on different testing days, using different pieces of exercise equipment, subjects are able to self-select a consistent intensity based on RPE. It may be prudent, however, to provide more specific information about when and how exercisers should feel fatigued (i.e., at the end of exercise your heart should be racing and breathing should be difficult) and to divide exertion into both central and peripheral components to encourage an adequate intensity to stress both systems.

The unique aspect of this study was the combination of both physiological responses with consumer opinions of various exercise modes. While the results of the questionnaires 
revealed that all pieces of aerobic exercise equipment were equally well liked, it is interesting and important to acknowledge that only $65 \%$ of participants with TP and $68 \%$ of those with PP were willing to use ALRS, whereas $100 \%$ were willing and able to use ACE. Even among participants willing to use the hybrid equipment, perceived safety was significantly less among participants with TP than for those with PP across all equipment types and was lower when comparing aerobic hybrid exercise to arm-only exercise regardless of injury level. The reasons cited for not wanting to use the hybrid exercise equipment was the associated transfer or being unable to properly secure feet to the machine. Considering there was no cardiovascular or metabolic advantage to using hybrid exercise, it may be important to promote the use of arm-only exercise, because it appears to be palatable to a broader scope of individuals with SCI.

Consumer opinions about the resistance exercise equipment were similar, as there were no differences between equipment types in terms of perceived enjoyment. Roughly all participants in both groups were willing to use both types of equipment; however, perceived safety was greater among participants with PP for both modes (WS and WP) than it was for those with TP. This group difference is likely reflective of the need for assistance in completing many of the resistance exercise manoeuvres (i.e., attaching wrist cuffs) and changing the equipment settings. It is difficult to distinguish an ideal method for resistance training on these findings alone, although the differences in the types of exercises that can be completed on each machine and the amount of weight that can be 
lifted would suggest a specific need for both equipment types in a comprehensive exercise prescription.

An obvious limitation to this study was that the metabolic assessments were only done in a subsample of the study participants. Another related limitation was the use of ACE to complete the peak exercise tests and to then use this $\mathrm{VO}_{2 \text { peak }}$ to compare the relative intensity of each exercise mode. Although the potential exists for differences in $\mathrm{VO}_{\text {2peak }}$ and $\mathrm{HR}_{\text {peak }}$ based on exercise mode, our finding that there were no differences in steady state submaximal $\mathrm{VO}_{2}$ or $\mathrm{HR}$ across the different modes suggests that there would also be no significant differences in peak values.

\section{Conclusion}

Passive hybrid exercise appears to provide no advantage in terms of metabolic or cardiovascular demand in people with SCI. Individuals with SCI are able to accurately attain a consistent exercise intensity using RPE on any type of exercise equipment, although may require specific instructions on how to assess central and peripheral fatigue. It may be ideal for health promotion strategies to promote the use of arm-only exercise, particularly among persons with TP because the transfer associated with hybrid machines can pose certain challenges. 


\section{References}

1. Ginis KAM, Hicks AL, Latimer AE, Warburton DER, Bourne C, Ditor DS, et al. The development of evidence-informed physical activity guidelines for adults with spinal cord injury. Spinal Cord 2011;49:1088-96.

2. Hicks AL, Martin Ginis KA, Pelletier CA, Ditor DS, Foulon B, Wolfe DL. The effects of exercise training on physical capacity, strength, body composition and functional performance among adults with spinal cord injury: a systematic review. Spinal Cord 2011;49:1103-27.

3. Buchholz AC, McGillivray CF, Pencharz PB. Differences in resting metabolic rate between paraplegic and able-bodied subjects are explained by differences in body composition. Am J Clin Nutr 2003;77:371-8.

4. Spungen AM, Adkins RH, Stewart CA, Wang J, Pierson RN, Waters RL, et al. Factors influencing body composition in persons with spinal cord injury: a crosssectional study. J Appl Physiol 2003;95:2398-407.

5. Groah SL, Nash MS, Ward EA, Libin A, Mendez AJ, Burns P, et al. Cardiometabolic risk in community-dwelling persons with chronic spinal cord injury. J Cardiopulm Rehabil Prev 2011;31:73-80.

6. Monroe MB, Tataranni PA, Pratley R, Manore MM, Skinner JS, Ravussin E. Lower daily energy expenditure as measured by a respiratory chamber in subjects with spinal cord injury compared with control subjects. Am J Clin Nutr 1998;68:1223-7.

7. Buchholz AC, Pencharz PB. Energy expenditure in chronic spinal cord injury. Curr Opin Clin Nutr Metab Care 2004;7:635-9.

8. Collins EG, Gater D, Kiratli J, Butler J, Hanson K, Langbein WE. Energy cost of physical activities in persons with spinal cord injury. Med Sci Sports Exerc 2010;42:691-700.

9. Taylor JA, Picard G, Widrick JJ. Aerobic capacity with hybrid FES rowing in spinal cord injury: comparison with arms-only exercise and preliminary findings with regular training. PMRJ 2011;3:817-24.

10. Brurok B, Tørhaug T, Karlsen T, Leivseth G, Helgerud J, Hoff J. Effect of lower extremity functional electrical stimulation pulsed isometric contractions on arm cycling peak oxygen uptake in spinal cord injured individuals. J Rehabil Med 2013;45:254-9. 
PhD Thesis - Chelsea A. Pelletier - McMaster University - Kinesiology

11. Hasnan N, Ektas N, Tanhoffer AIP, Tanhoffer R, Fornusek C, Middleton J, et al. Exercise responses during FES cycling in individuals with spinal cord injury. Med Sci Sports Exerc 2013;45:1131-8.

12. Borg G. Psychophysical bases of perceived exertion. Med Sci Sports Exerc 1982;14:377-81.

13. Lewis JE, Nash MS, Hamm LF, Martins SC, Groah SL. The relationship between perceived exertion and physiologic indicators of stress during graded arm exercise in persons with spinal cord injuries. Arch Phys Med Rehabil 2007;88:1205-11.

14. Cowan RE, Ginnity KL, Kressler J, Nash MS. Assessment of the talk test and rating of perceived exertion for exercise intensity prescription in persons with paraplegia. Top Spinal Cord Inj Rehabil 2012;18:212-9.

15. Hooker SP, Greenwood JD, Hatae DT, Husson RP, Matthiesen TL, Waters AR. Oxygen uptake and heart rate relationship in persons with spinal cord injury. Med Sci Sports Exerc 1993;25:1115-9.

16. Goosey-Tolfrey V, Lenton J, Goddard J, Oldfield V, Tolfrey K, Eston R. Regulating intensity using perceived exertion in spinal cord-injured participants. Med Sci Sports Exerc 2010;42:608-13.

17. Maki KC, Langbein WE, Reid-Lokos C. Energy cost and locomotive economy of handbike and rowcycle propulsion by persons with spinal cord injury. JRRD 1995;32:170-8.

18. Hol AT, Eng JJ, Miller WC, Sproule S, Krassioukov AV. Reliability and validity of the six-minute arm test for the evaluation of cardiovascular fitness in people with spinal cord injury. Arch Phys Med Rehabil 2007;88:489-95.

19. Pelletier CA, Jones G, Latimer A, Warburton DE, Hicks AL. Aerobic capacity, orthostatic tolerance, and exercise perceptions at discharge from inpatient spinal cord injury rehabilitation. Arch Phys Med Rehabil 2013, doi:10.1016/j.apmr.2013.05.011

20. Cleeland CS, Ryan KM. Pain assessment: global use of the Brief Pain Inventory. Ann Acad Med Singap 1994;23:129-38.

21. Raichle KA, Osborne TL, Jensen MP, Cardenas D. The reliability and validity of pain interference measures in persons with spinal cord injury. J Pain 2006;7:179_ 86.

22. Nunnally JC. Psychometric theory. 2nd ed. New York: McGraw-Hill; 1978. 
PhD Thesis - Chelsea A. Pelletier - McMaster University - Kinesiology

23. Pickett GE, Campos-Benitez M, Keller JL, Duggal N. Epidemiology of traumatic spinal cord injury in Canada. Spine 2006;31:799-805. 
PhD Thesis - Chelsea A. Pelletier - McMaster University - Kinesiology

Table 1. Participant Characteristics

\begin{tabular}{lccc}
\hline Characteristic & Overall & TP & PP \\
\hline Sample Size $(n)$ & 36 & 17 & 19 \\
Age (years) & $41.1 \pm 12.2$ & $40.6 \pm 14.2$ & $41.5 \pm 10.4$ \\
Sex & 29 males, 7 females & 11 males, 6 females & 18 males, 1 female \\
Level of Injury & C3 - T11 & C $3-$ C8 & T3 - T12 \\
Time Post Injury (years) & $11.4 \pm 10.7$ & $13.2 \pm 12.0$ & $9.89 \pm 9.22$ \\
\hline
\end{tabular}

Values are mean \pm SD.

Abbreviations: TP, tetraplegia; PP, paraplegia 
PhD Thesis - Chelsea A. Pelletier - McMaster University - Kinesiology

Table 2. Results of the Peak Exercise Test

\begin{tabular}{|c|c|c|}
\hline Variable & TP $(n=5)$ & $\mathbf{P P}(n=4)$ \\
\hline Body Mass (kg) & $85.1 \pm 18.2$ & $80.5 \pm 26.4$ \\
\hline \multicolumn{3}{|l|}{$\mathrm{VO}_{2}(\mathrm{~L} / \mathrm{min})$} \\
\hline Resting & $0.29 \pm 0.10$ & $0.29 \pm 0.07$ \\
\hline Peak & $1.31 \pm 0.45$ & $1.76 \pm 0.37$ \\
\hline \multicolumn{3}{|l|}{$\mathrm{VO}_{2}\left(\mathrm{~mL} \cdot \mathrm{kg}^{-1} \cdot \mathrm{min}^{-1}\right)$} \\
\hline Resting & $3.38 \pm 0.57$ & $3.75 \pm 0.52$ \\
\hline Peak & $15.1 \pm 3.35$ & $22.8 \pm 4.81 *$ \\
\hline $\mathrm{RER}_{\text {peak }}$ & $1.00 \pm 0.09$ & $1.09 \pm 0.03$ \\
\hline $\mathrm{VE}_{\text {peak }}(\mathrm{L} / \mathrm{min})$ & $54.1 \pm 20.6$ & $53.1 \pm 34.3$ \\
\hline \multicolumn{3}{|l|}{$\operatorname{HR}(\mathrm{bpm})$} \\
\hline Resting & $72.6 \pm 16.8$ & $73.0 \pm 10.1$ \\
\hline Peak & $129 \pm 15.9$ & $144 \pm 24.9$ \\
\hline $\mathrm{PO}_{\mathrm{pek}}(\mathrm{W})$ & $53.6 \pm 30.5$ & $100 \pm 9.13^{*}$ \\
\hline
\end{tabular}

Values are mean $\pm \mathrm{SD}, *$ indicates a significant difference between groups $(\mathrm{p}<0.05)$

Abbreviations: $\mathrm{VO}_{2}$, oxygen uptake; RER, respiratory exchange ratio; VE, minute ventilation; $\mathrm{HR}$, heart rate; $\mathrm{PO}$, power output; TP, tetraplegia; PP, paraplegia 
PhD Thesis - Chelsea A. Pelletier - McMaster University - Kinesiology

Table 3. Physiological Variables during 20-Minutes of Steady State Submaximal Exercise

\begin{tabular}{lcccc}
\hline Variable & & ACE & AG & ALRS \\
& & & & \\
\hline Steady State HR $(\mathrm{bpm})$ & TP & $104.6 \pm 15.5$ & $102.0 \pm 12.9$ & $97.6 \pm 10.5$ \\
& PP & $122.8 \pm 24.2$ & $128.3 \pm 20.8$ & $123.8 \pm 18.6$ \\
$\% \mathrm{HR}_{\text {peak }}$ & & & & \\
& TP & $81.6 \pm 10.1$ & $79.9 \pm 11.4$ & $76.4 \pm 9.40$ \\
Steady State $\mathrm{VO}_{2}(\mathrm{~L} / \mathrm{min})$ & PP & $85.2 \pm 3.73$ & $89.6 \pm 7.24$ & $86.4 \pm 6.40$ \\
& & & & \\
Steady State $\mathrm{VO}_{2}\left(\mathrm{~mL} \cdot \mathrm{kg}^{-1} \cdot \mathrm{min}^{-1}\right)$ & PP & $0.56 \pm 0.31$ & $0.52 \pm 0.19$ & $0.60 \pm 0.29$ \\
& TP & $6.49 \pm 3.19$ & $0.91 \pm 0.29$ & $0.80 \pm 0.28$ \\
& PP & $10.1 \pm 1.99$ & $11.4 \pm 1.57$ & $10.1 \pm 1.23$ \\
& & & & \\
& TP & $41.8 \pm 12.8$ & $40.8 \pm 7.74$ & $46.2 \pm 14.9$ \\
& PP & $44.4 \pm 1.45$ & $50.8 \pm 6.77$ & $45.5 \pm 10.8$ \\
\hline
\end{tabular}

Values are means $\pm \mathrm{SD}$

Abbreviations: ACE, arm cycle ergometer; AG, arm glider; ALRS, arm and leg recumbent stepper; $\mathrm{HR}$, heart rate; $\mathrm{VO}_{2}$, oxygen uptake; $\mathrm{TP}$, tetraplegia; $\mathrm{PP}$, paraplegia 
PhD Thesis - Chelsea A. Pelletier - McMaster University - Kinesiology

Table 4. Ratings of Perceived Exertion (10-point scale) During 20-Minutes of Steady State Submaximal Exercise

\begin{tabular}{|c|c|c|c|c|}
\hline Time & $\overline{A C E}$ & $\overline{A G}$ & ALRS & $\overline{\text { ALCE }}$ \\
\hline \multicolumn{5}{|l|}{$5 \mathrm{~min}$} \\
\hline $\mathrm{TF}$ & $2.5 \pm 1.46$ & $3.2 \pm 0.79$ & $3.2 \pm 1.17$ & $3.5 \pm 0.71$ \\
\hline PF & $3.1 \pm 1.41$ & $2.9 \pm 0.64$ & $3.0 \pm 0.85$ & $4.3 \pm 1.03$ \\
\hline \multicolumn{5}{|l|}{$10 \mathrm{~min}$} \\
\hline $\mathrm{TF}$ & $3.9 \pm 1.52$ & $3.9 \pm 1.10$ & $4.5 \pm 1.13$ & $3.5 \pm 0.71$ \\
\hline $\mathrm{PF}$ & $3.7 \pm 1.54$ & $3.5 \pm 0.76$ & $3.6 \pm 0.79$ & $4.8 \pm 1.33$ \\
\hline \multicolumn{5}{|l|}{$15 \mathrm{~min}$} \\
\hline $\mathrm{TF}$ & $4.4 \pm 1.45$ & $4.4 \pm 1.35$ & $5.0 \pm 1.41$ & $3.5 \pm 0.71$ \\
\hline PF & $4.1 \pm 1.41$ & $4.0 \pm 1.15$ & $4.3 \pm 0.97$ & $4.6 \pm 0.89$ \\
\hline \multicolumn{5}{|c|}{$20 \mathrm{~min}$} \\
\hline $\mathrm{TF}$ & $5.6 \pm 2.33$ & $5.6 \pm 2.45$ & $6.7 \pm 2.00$ & $3.5 \pm 0.71$ \\
\hline PF & $4.6 \pm 1.50$ & $4.4 \pm 0.98$ & $4.8 \pm 0.98$ & $5.2 \pm 0.84$ \\
\hline
\end{tabular}

Values are mean $\pm \mathrm{SD}$.

Abbreviations: ACE, arm cycle ergometer; AG, arm glider; ALRS, arm and leg recumbent stepper; ALCE, arm and leg cycle ergometer; TP, tetraplegia; PP, paraplegia 
PhD Thesis - Chelsea A. Pelletier - McMaster University - Kinesiology

Table 5. Results of Consumer Preference Questionnaire

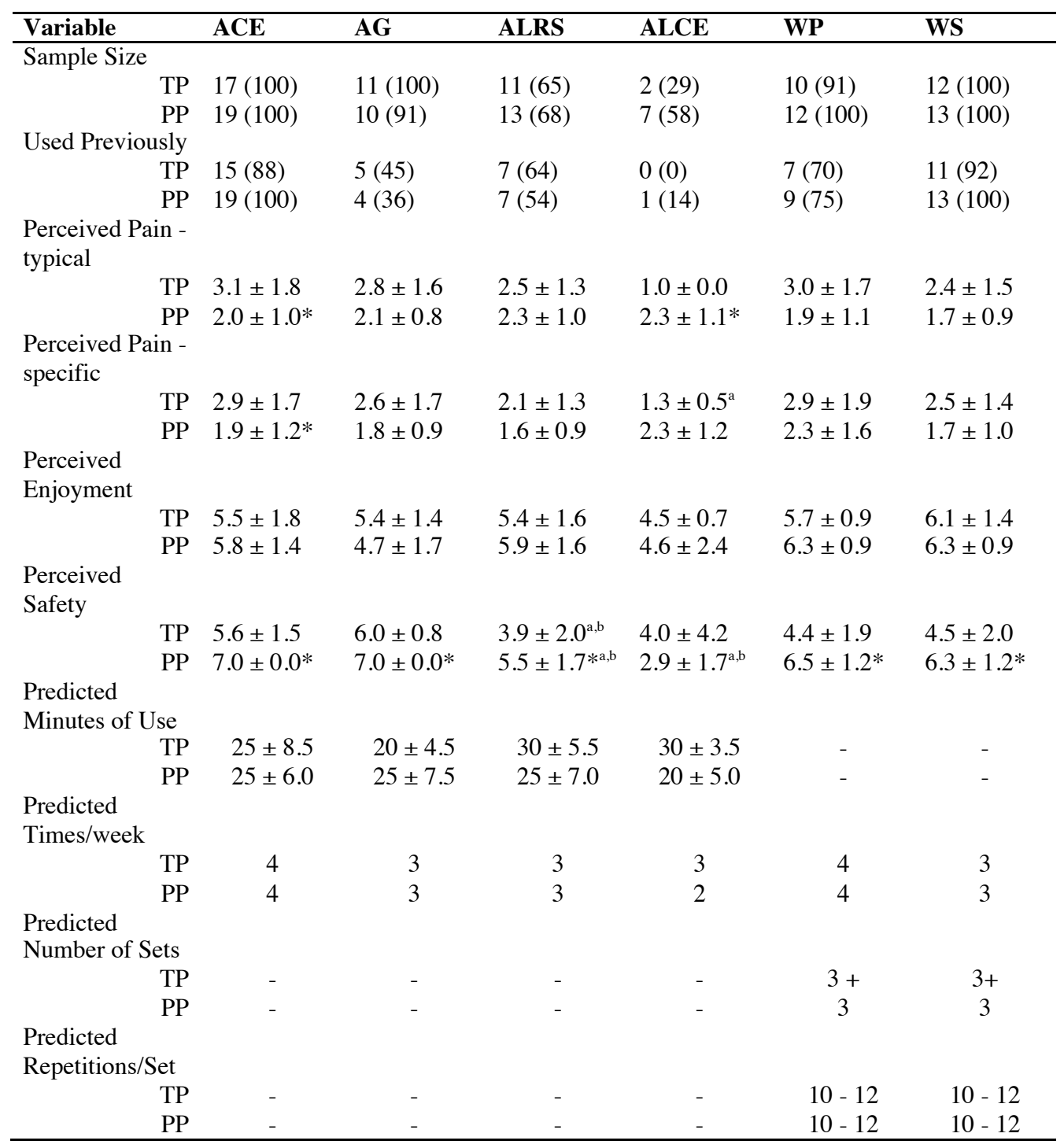

Values are presented as $n$ (\% of eligible participants) or mean composite score \pm SD.

Note: Pain, enjoyment, and safety measures are scored out of $7(1=$ none at all/not at all confident, $7=\mathrm{a}$ lot $/$ completely confident)

Abbreviations: ACE, arm cycle ergometer; AG, arm glider; ALRS, arm and leg recumbent stepper; ALCE, arm and leg cycle ergometer; WP, wall pulleys; WS, weight stack; TP, tetraplegia; $\mathrm{PP}$, paraplegia

* indicates a significant difference between groups (TP vs. PP; $p<0.05)$.

${ }^{a}$ indicates a significant difference compared to ACE $(\mathrm{p}<0.05)$

${ }^{\mathrm{b}}$ indicates a significant differences compared to AG $(\mathrm{p}<0.05)$ 

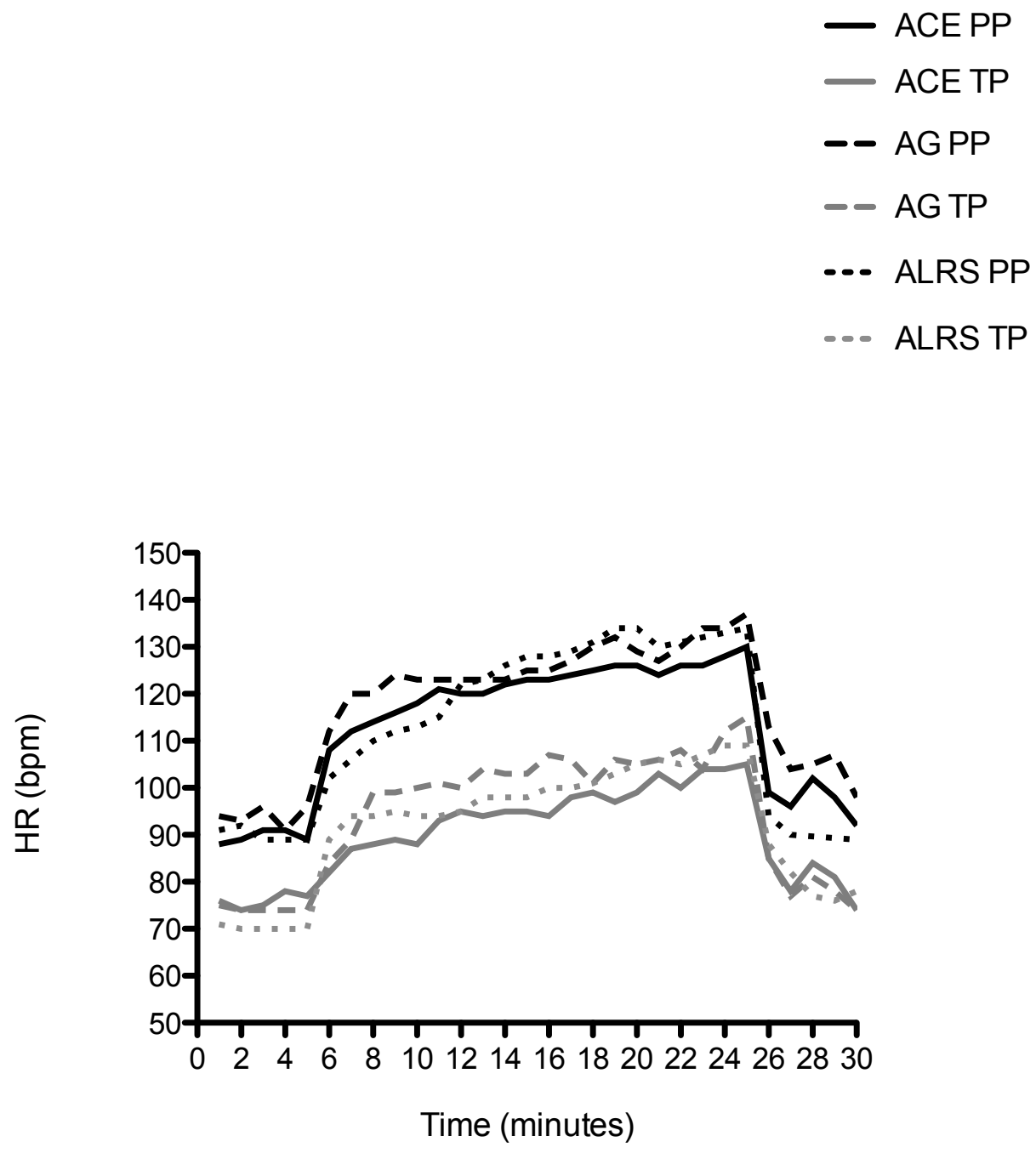

Figure 1. Heart Rate during 20-Minutes of Steady State Submaximal Exercise in Participants with Tetraplegia (TP) and Paraplegia (PP) 

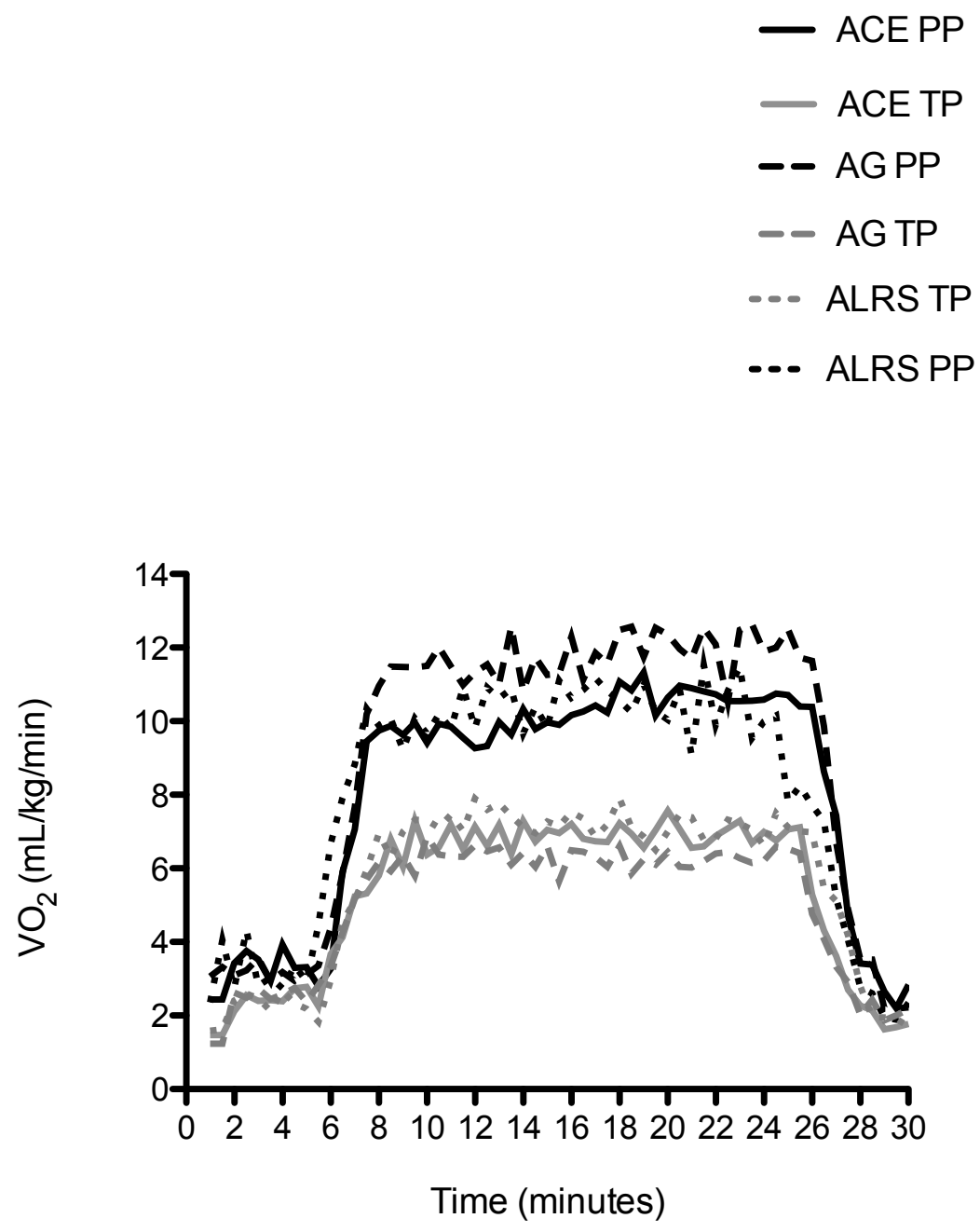

Figure 2. Oxygen Consumption during 20-Minutes of Steady State Submaximal Exercise in Participants with Tetraplegia (TP) and Paraplegia (PP) 


\title{
Chapter 5
}

\section{Implementation of the Physical Activity Guidelines for Adults with Spinal Cord Injury: Effects on Aerobic Capacity and Muscle Strength}

\author{
Authors: Chelsea A. Pelletier, Julia Totosy de Zepetnek, Maureen MacDonald, \\ Audrey L. Hicks
}


Study Design: Randomized controlled trial

Objectives: To evaluate the effectiveness of the physical activity guidelines (PAG) for adults with spinal cord injury for improving aspects of fitness in a community cohort. Setting: Community exercise program for adults with chronic spinal cord injury Methods: Sixteen participants (age: $42.6 \pm 11.6$ years, C1-T11, $12.1 \pm 9.2$ years postinjury) were randomized into PAG training $(n=8)$ or active control $(\mathrm{CON}, \mathrm{n}=8)$ groups. PAG training was 2x/week for 16-weeks and involved at least 20 minutes of aerobic exercise at a moderate to vigorous intensity and 3 sets of 10 repetitions (at $50-70 \% 1$ repetition maximum; 1RM) using each major muscle group of the upper body. The CON group were members in a twice-weekly exercise program but were not provided with guidance to achieve a certain training intensity. Pre- and post-testing included a peak exercise and aerobic endurance test on an arm ergometer, 1RM testing, a muscle endurance test, and a questionnaire to evaluate consumer satisfaction with the PAG. Results: There was a significant (group $x$ time interaction; $p<0.05$ ) increase in peak aerobic capacity $(17.1 \%$ ) and power output (peak: $9.7 \%$, submaximal: $12.6 \%$ ) in the PAG group only. Increases in strength ranged from 9.8-46.0\% and were significantly different from $\mathrm{CON}(\mathrm{p}<0.05)$ for vertical bench press, seated row, and rickshaw press. Adherence to the exercise program was $87.9 \pm 7.9 \%$ for PAG, $48.7 \pm 34.4 \%$ for $\mathrm{CON}(\mathrm{p}<0.05)$. Participants in the PAG group were highly satisfied with both the aerobic and resistance aspects of the training. 
PhD Thesis - Chelsea A. Pelletier - McMaster University - Kinesiology

Conclusions: The PAG for adults with spinal cord injury are sufficient to improve aspects of aerobic and muscular fitness and should be promoted as a means to improve physical capacity.

Sponsorship: Ontario Neurotrauma Foundation

Keywords: Exercise, disability, health promotion, aerobic fitness, muscle strength 


\section{Introduction}

Individuals with spinal cord injury (SCI) are at increased risk for many lifestyle related illnesses due to the physical inactivity, changes in body composition, and obesity that are common in this population. Specifically, cardiovascular disease is the leading cause of mortality among persons with SCI, and the prevalence of metabolic syndrome is nearly double that of the able-bodied population. ${ }^{1,2}$ While the risk factors for cardiovascular disease have not been well established in this population, physical inactivity is an independent risk factor and has indirect effects through its impact on body mass index, glucose homeostasis, and blood lipids. Physical fitness is not only important for cardiovascular health but can be linked to many aspects of quality of life including return to work, ${ }^{3}$ functional ability, ${ }^{4}$ and activities of daily living. ${ }^{5}$ Thus, there is an urgent need for health promotion strategies to encourage the adoption of regular physical activity.

The physical activity guidelines for adults with SCI were released in 2011 in order provide clinicians and exercise professionals information from which to form an exercise prescription. The guidelines recommend twice weekly exercise involving aerobic exercise at a moderate to vigorous intensity for at least 20 minutes, and resistance exercise of 3 sets of 10 repetitions using each major muscle group. ${ }^{6}$ These guidelines consider not only scientific evidence previously shown to improve aspects of fitness but also the considerable barriers to exercise participation among individuals with a physical disability. ${ }^{7}$ 
There is strong evidence to support the efficacy of exercise to improve aspects of physical fitness in the SCI population. Improvements in cardiovascular fitness have been demonstrated following aerobic exercise training using arm cycle ${ }^{8,9}$ or wheelchair ${ }^{10,11}$ ergometry, and improvements in muscle strength have been demonstrated with both neuromuscular electrical stimulation ${ }^{12}$ and voluntary muscle contraction training protocols. ${ }^{13}$ Hardly any studies have demonstrated the efficacy of combined aerobic and resistance exercise training programs. A 9-month training protocol of combined resistance (2-3 sets of 50-80\% 1 repetition maximum; 1RM) and aerobic exercise $(70 \%$ maximum heart rate or 3-4 on rating of perceived exertion (RPE) scale) resulted in an increase in submaximal power output of $81 \%$, and a $19-34 \%$ increase in muscle strength. ${ }^{14}$ Circuit training (arm cycle ergometry and resistance training done in sequence) has also resulted in improvements of $10.4 \%$ in peak aerobic capacity and an increase in muscle strength of 38.6-59.7\% after 4-months of training three times per week. ${ }^{15}$ In a shorter circuit training protocol lasting 10-weeks, improvements were seen in peak aerobic capacity $(29.7 \%)$ and strength $(11.9-30 \%) .{ }^{16}$ Now that the physical activity guidelines have been released, an important next step is to implement the guidelines in a practical community setting over a defined time period and to establish their validity in improving aspects of physical fitness in adults with chronic SCI.

Thus, the purpose of this study was to evaluate the efficacy of the physical activity guidelines for adults with SCI for improving aspects of fitness in a community-based sample. It was predicted that 16-weeks of training following the physical activity 
guidelines would result in significant increases in aerobic fitness and muscle strength when compared to an active control group.

\section{Methods}

\section{Participants}

Participants with chronic SCI (> 1 year post injury), who were 18-65 years, wheelchair dependent, and able to understand English were recruited to participate from April 2012June 2013 through advertisements at local community organizations. Exclusion criteria included any progressive loss of neurologic function within the previous six months. The Hamilton Health Sciences Research Ethics board approved the study protocol, and informed written consent was obtained from each participant. After baseline testing was completed, participants were randomized into a physical activity guidelines training (PAG) or active control (CON) group using computer software (GraphPad Software, Inc., La Jolla, California, United States).

\section{Peak Aerobic Capacity}

To measure peak oxygen consumption $\left(\mathrm{VO}_{2 \text { peak }}\right)$, participants performed a symptomlimited graded arm cycle ergometer test (Lode B.V, Groningen, the Netherlands). Resistance was increased by $5 \mathrm{~W} / \mathrm{min}$ for participants with tetraplegia and $10 \mathrm{~W} / \mathrm{min}$ for 
participants with paraplegia. ${ }^{17,18}$ In some cases, this protocol was modified slightly to ensure the test was 8-12 minutes in duration. Expired gas and ventilatory parameters were acquired throughout the protocol using the Moxus Metabolic System (AEI Technologies, Inc., Bastrop, Texas, United States) allowing for the determination of $\mathrm{VO}_{2 \text { peak }}$. Central (i.e., breathing) and peripheral (i.e., arm fatigue) RPE (Borg 10-point scale) ${ }^{19}$ were assessed every minute. Continuous measurements of heart rate (HR) were recorded throughout the protocol (Polar Electro Canada, Lachine, Quebec, Canada). $\mathrm{VO}_{2 \text { peak }}$, $\mathrm{HR}_{\text {peak }}$, and peak minute ventilation $\left(\mathrm{VE}_{\text {peak }}\right)$ were defined as the greatest value achieved in a 30-second interval. Peak power output $\left(\mathrm{PO}_{\text {peak }}\right)$ was defined as the greatest resistance achieved and maintained for at least 15 -seconds.

Aerobic Endurance

Participants were asked to complete an aerobic endurance test on an arm cycle ergometer (Lode B.V, Groningen, the Netherlands). After a 1-minute warm-up, resistance (W) was set to the corresponding value achieved at $70 \%$ of $\mathrm{VO}_{2 \text { peak }}$. $\mathrm{HR}$ (Polar Electro Canada, Lachine, Quebec, Canada) and $\mathrm{VO}_{2}$ (Moxus Metabolic System, AEI Technologies, Inc., Bastrop, Texas, United States) were continuously monitored. Participants were instructed to continue until volitional fatigue and were stopped if they reached 20:00 minutes or were unable to maintain at least 40 revolutions per minute. 
Muscle Strength

Muscle strength testing was completed on a multi-station wheelchair accessible weight training system (Equalizer Exercise Machines, Red Deer, Alberta, Canada) or unilateral wall pulleys (Endorphin Pulleys, Patterson Medical Canada, Mississauga, Ontario, Canada). 1RM was assessed in a variety of upper body exercises: latissimus pull-down, vertical bench press, seated row, bicep curl (right and left), and rickshaw press. The order of exercises was standardized, and there was sufficient rest between exercises to minimize the potential influence of developing muscle fatigue.

\section{Muscle Endurance}

As part of post-testing, participants completed an assessment of muscle endurance. This test involved lifting each of the pre-training exercises for as many repetitions as possible using the pre-training 1RM.

\section{Training Intervention}

Participants in the PAG group completed a 16-week progressive exercise program that followed the physical activity guidelines for adults with SCI. The twice-weekly program consisted of 20 minutes of aerobic exercise at a moderate to vigorous intensity (RPE 3-6 on 10-point scale) and 3 sets of 10 repetitions (50-70\% 1RM) of resistance exercise using 
each major muscle group of the upper body. The aerobic exercise equipment used was an arm cycle ergometer (Monark Arm Ergometer, Patterson Medical Supply Inc., Mississauga, Ontario, Canada), arm glide ergometer (Vitaglide, RMT Fitness, Miami, Florida, United States), or hybrid recumbent stepper (NuStep T5XR Recumbent Cross Trainer, NuStep, Inc., Ann Arbor, Michigan, United States) as per the choice of the participant. Resistance exercise was completed using a combination of multi-station accessible weight stack equipment (Equalizer Exercise Machines, Red Deer, Alberta, Canada), wall pulleys (Endorphin Pulleys, Patterson Medical Canada, Mississauga, Ontario, Canada), and free weights.

Participants in the CON group were members in a twice-weekly community exercise program geared for adults with SCI, but were given no specific guidance or encouragement with respect to attaining the minimum exercise intensity associated with the physical activity guidelines.

\section{Adherence}

Adherence was calculated based on the percentage of a maximum of 32 sessions (2x/week for 16 -weeks). If participants in the PAG group missed any sessions during the training period they were given 4-weeks to complete the additional sessions. Adherence rates are presented as $i$ ) during the 16-week period only (for comparison with $\mathrm{CON}$ ) and ii) total number of sessions completed (including make-up sessions). 
Satisfaction with the Guidelines

A questionnaire to assess consumer satisfaction with the physical activity guidelines was administered to participants in the PAG group at the end of the training protocol. All questions were rated from $1=$ strongly disagree to $7=$ strongly agree, and mean composite scores were calculated for each set of items. Satisfaction with aerobic exercise $(\alpha=0.84)$ and resistance exercise $(\alpha=0.91)$ was evaluated with five scales each, asking whether the participants felt the training program was appropriate in terms of time, intensity, ability, if it was enjoyable, and if it improved physical fitness. Perception of pain was measured with one item: "I was able to complete the exercises without any additional pain or discomfort." Enjoyment was also measured with one item: "Overall, I enjoyed the exercise program."

\section{Data Analysis}

Data were analyzed using Microsoft Excel (Microsoft Canada, Mississauga, Ontario, Canada) and IBM SPSS (Version 20.0, SPSS Inc., Chicago, Illinois, United States) software. Participant demographic information, adherence rates, and pre-testing data were compared between groups using a one-way analysis of variance or the Welch F-test where the assumption of equal variances was violated. Pre- and Post-training values were compared between groups using a two-way (group x time) repeated measures analysis of variance. 


\section{Results}

\section{Participants}

Sixteen participants (age 42.6 \pm 11.6 years, C1-T11, $12.1 \pm 9.2$ years post injury) were recruited to participate; 8 were randomized into the PAG group and 8 into the CON group. Two participants in the CON group did not complete the study: one dropped out immediately following randomization due to dissatisfaction with group allocation, and one did not complete post-test due to the re-occurrence of a chronic shoulder injury (not study-related). There were no significant differences in demographic information between groups, and this information is presented in table 1 . The most common causes of injury were motor vehicle accidents (37.5\%), followed by sports and recreation accidents (25\%), non-traumatic $(25 \%)$, fall $(6.3 \%)$, and surgery $(6.3 \%)$, which are consistent with national averages. $^{20}$

\section{Peak Aerobic Capacity}

Results of the peak exercise test are presented in table 2. There were no significant differences between groups at baseline. There was a significant increase in relative $\mathrm{VO}_{2 \text { peak }}($ group $\mathrm{x}$ time interaction; $\mathrm{p}=0.03$ ) in the PAG group following training. There was also a significant increase in $\mathrm{PO}_{\text {peak }}$ in the PAG group (group $\mathrm{x}$ time interaction; $\mathrm{p}=$ 
PhD Thesis - Chelsea A. Pelletier - McMaster University - Kinesiology

0.047). The $\mathrm{CON}$ group experienced no change in either $\mathrm{VO}_{2 \text { peak }}$ or $\mathrm{PO}_{\text {peak }}$ over the 16week period.

Aerobic Endurance

There were no significant differences between groups at baseline for any of the variables (table 3). Post-training, the PAG group completed the submaximal test at a higher PO than the CON group (group $\mathrm{x}$ time interaction, $\mathrm{p}=0.02$ ), despite similar submaximal HR and $\mathrm{VO}_{2}$. While there was an increase in performance time for both PAG (+2:07 min) and CON (+ 1:23 min) groups, these increases did not reach significance.

Muscle Strength

Changes in muscle strength are presented in table 4 . There were no significant differences between PAG and CON at baseline. There was a significant group $\mathrm{x}$ time main effect for vertical bench press $(p=0.048)$, seated row $(p=0.04)$, and rickshaw press $(p=0.03)$, reflective of mean strength increases in the PAG group of $7.1 \mathrm{~kg}, 8.7 \mathrm{~kg}$, and $27.4 \mathrm{~kg}$, respectively. 


\section{Muscle Endurance}

The muscle endurance test results are presented in figure 1. The PAG group was able to lift more repetitions of the pre-training 1RM for all manoeuvres except biceps curl; however, the group differences were only significant for vertical bench press $(p=0.01)$.

\section{Adherence}

Mean adherence rates during the 16-week training period were $87.9 \pm 7.7 \%$ (range 75 $100 \%)$ among participants in the PAG group and $48.7 \pm 34.4 \%$ in CON $(\mathrm{p}=0.02)$. When the make-up sessions were included, mean adherence was $98.4 \pm 3.3 \%$ in the PAG group. The most prevalent barriers were personal health issues $(80 \%)$, other commitments $(18.8 \%)$, transportation (12.5\%), medical appointments $(12.5 \%)$, and vacation $(6.3 \%)$ among participants in the PAG group. The barriers most likely to prevent attendance were personal health issues (15), followed by transportation (6), other commitments (6), medical appointments (3), and vacation (1).

\section{Satisfaction with the Guidelines}

Satisfaction with both the aerobic (mean: $6.3 \pm 0.73$ ) and resistance (mean: $6.6 \pm 0.6$ ) aspects of the PAG and the training protocol were very high (maximum score of 7). 
Enjoyment of the exercise program was also high (mean: $6.9 \pm 0.38$, maximum score of 7). Mean score for perceived pain was $5.4 \pm 1.8$, with a maximum score of 7 .

\section{Discussion}

The purpose of this study was to evaluate the efficacy of the physical activity guidelines for adults with SCI to improve aspects of physical fitness over a defined time period in a sample of individuals living in the community. The findings suggest a significant improvement in physical capacity in terms of both $\mathrm{VO}_{2 \text { peak }}(+17.1 \%)$ and $\mathrm{PO}_{\text {peak }}(+9.7 \%)$. Strength gains ranged from $9.8-46.0 \%$ and were statistically significant for vertical bench press $(+14.4 \%)$, seated row $(+20.4 \%)$, and rickshaw press $(+46.0 \%)$ manoeuvres. Adherence rate for the prescribed 16-week exercise program was excellent (mean: 87.9\%, range: $75-100 \%$ ), and participants were highly satisfied with the training program.

The improvements observed in physical capacity in the present study are consistent with previous studies, which report a range in $\mathrm{VO}_{2 \text { peak }}$ increases from 8.6-29.7\% following training programs incorporating aerobic and/or resistance exercise..$^{10,15,16,21}$ This finding is promising given that the current protocol involved exercise only twice per week, at a selfselected intensity (moderate-vigorous, RPE 3-6) and exercise mode. Therefore, given the correct instruction and equipment, individuals are able to (or should be able to) select an appropriate exercise intensity (based on a defined RPE) to improve physical fitness. 
The increase in submaximal PO during the aerobic endurance test in the PAG group $(+12.6 \%)$ indicates that participants were able perform significantly more work at the same HR and RPE. Similar results have been reported in previous training studies and are likely reflective of both increases in muscle strength and cardiovascular capacity. ${ }^{10,14}$ The absence of any training effect on submaximal performance time may be explained by the insensitivity of the testing protocol. PO for this assessment was set based on the corresponding value achieved at $70 \% \mathrm{VO}_{2 \text { peak }}$, and several participants (6/14) were able to achieve the maximum of 20 minutes during baseline testing. While this performance time may indicate a relatively high level of fitness in our participants, it may also be reflective of the difficulties in obtaining a true assessment of $\mathrm{VO}_{2 \text { peak }}$ in this population. RPE values indicate that, on average, participants stopped the peak exercise test based on peripheral (i.e., arm or shoulder) fatigue, thus the PO selected may have been an underestimation.

The improvements in muscle strength in the present study are similar to other improvements involving voluntary muscle strength training. ${ }^{14-16}$ The fact that increases in $1 \mathrm{RM}$ and muscle endurance were present for all manoeuvres, but only statistically significant compared to $\mathrm{CON}$ for some muscle groups, is likely reflective of the high degree of individual variability and voluntary muscle function.

The utilization of an active CON group was both a strength and limitation of this study. While it is true that an inactive CON group would have provided the best opportunity to evaluate the effectiveness of the physical activity guidelines, using a control group that 
was recreationally active at the same frequency per week as our intervention group could be more representative of a community sample. The adherence rate of the CON group was quite variable (mean: $48.7 \pm 34.4 \%$, range: $0-93.8 \%$ ) compared to the PAG group (mean: $87.9 \pm 7.7 \%$, range: $75-100 \%$ ), suggesting there was likely a significant amount of motivation provided by participating in the study and experiencing the one-on-one attention during training. Similarly, Ditor et $a l^{22}$ reported a significant drop in adherence rates from $80.6 \%$ to $42.7 \%$ in just 3-months following a 9-month training study. The high adherence rate can also be attributed to the fact that participation in the present study eliminated some of the most commonly reported barriers to exercise participation in the SCI population: resource and facility accessibility, program cost, knowledge about exercise, and equipment accessibility. ${ }^{23-25}$ This emphasizes the efficacy of creating personalized training opportunities and fitness facilities specific to individuals with SCI to encourage sustained participation.

\section{Conclusions}

The results of this study establish the effectiveness of implementing the physical activity guidelines for adults with SCI living in the community and demonstrate their efficacy for improving physical fitness. High adherence rates and satisfaction with the training program indicate that these guidelines are well received by the consumer and should thus be incorporated into various health promotion strategies. Future studies should now be undertaken to determine if following the guidelines would also result in improved 
PhD Thesis - Chelsea A. Pelletier - McMaster University - Kinesiology

cardiovascular health or a decrease in cardiovascular disease risk factors in this population. 


\section{References}

1. Garshick E, Kelley A, Cohen SA, Garrison A, Tun CG, Gagnon D, et al. A prospective assessment of mortality in chronic spinal cord injury. Spinal Cord 2005;43:408-16.

2. Lee MY, Myers J, Hayes A, Madan S, Froelicher VF, Perkash I, et al. C-reactive protein, metabolic syndrome, and insulin resistance in individuals with spinal cord injury. J Spinal Cord Med 2005;28:20-5.

3. Noreau L, Shephard RJ. Return to work after spinal cord injury: the potential contribution of physical fitness. Paraplegia 1992;30:563-72.

4. Dallmeijer AJ, van der Woude LHV. Health related functional status in men with spinal cord injury: relationship with lesion level and endurance capacity. Spinal Cord 2001;39:577-83.

5. Janssen TWJ, van Oers CAJM, Veeger HEJ, Hollander AP, van der Woude LHV, Rozendal RH. Relationship between physical strain during standardised ADL tasks and physical capacity in men with spinal cord injuries. Paraplegia 1994;32:844-59.

6. Ginis KAM, Hicks AL, Latimer AE, Warburton DER, Bourne C, Ditor DS, et al. The development of evidence-informed physical activity guidelines for adults with spinal cord injury. Spinal Cord 2011;49:1088-96.

7. Hicks AL, Martin Ginis KA, Pelletier CA, Ditor DS, Foulon B, Wolfe DL. The effects of exercise training on physical capacity, strength, body composition and functional performance among adults with spinal cord injury: a systematic review. Spinal Cord 2011;49:1103-27.

8. El-Sayed MS, Younesian A. Lipid profiles are influenced by arm cranking exercise and training in individuals with spinal cord injury. Spinal Cord 2004;43:299-305.

9. Bizzarini E, Saccavini M, Lipanje F, Magrin P, Malisan C, Zampa A. Exercise prescription in subjects with spinal cord injuries. Arch Phys Med Rehabil 2005;86:1170-5.

10. Bougenot M-P, Tordi N, Betik AC, Martin X, Le Foll D, Parratte B, et al. Effects of a wheelchair ergometer training programme on spinal cord-injured persons. Spinal Cord 2003;41:451-6.

11. Hooker SP, Wells CL. Effects of low- and moderate-intensity training in spinal cord-injured persons. Med Sci Sports Exerc 1989;21:18-21. 
PhD Thesis - Chelsea A. Pelletier - McMaster University - Kinesiology

12. Sabatier MJ, Stoner L, Mahoney ET, Black C, Elder C, Dudley GA, et al. Electrically stimulated resistance training in SCI individuals increases muscle fatigue resistance but not femoral artery size or blood flow. Spinal Cord $2005 ; 44: 227-33$.

13. Jayaraman A, Thompson CK. Short-term maximal-intensity resistance training increases volitional function and strength in chronic incomplete spinal cord injury: A pilot study. JNPT 2013, doi:10.1097/NPT.0b013e31828390a1.

14. Hicks AL, Martin KA, Ditor DS, Latimer AE, Craven C, Bugaresti J, et al. Longterm exercise training in persons with spinal cord injury: effects on strength, arm ergometry performance and psychological well-being. Spinal Cord 2003;41:34-43.

15. Nash MS, van de Ven I, van Elk N, Johnson BM. Effects of circuit resistance training on fitness attributes and upper-extremity pain in middle-aged men with paraplegia. Arch Phys Med Rehabil 2007;88:70-5.

16. Jacobs PL, Nash MS, Rusinowski JW. Circuit training provides cardiorespiratory and strength benefits in persons with paraplegia. Med Sci Sports Exerc 2001;33:711-7.

17. Hol AT, Eng JJ, Miller WC, Sproule S, Krassioukov AV. Reliability and validity of the six-minute arm test for the evaluation of cardiovascular fitness in people with spinal cord injury. Arch Phys Med Rehabil 2007;88:489-95.

18. Pelletier CA, Jones G, Latimer A, Warburton DE, Hicks AL. Aerobic capacity, orthostatic tolerance, and exercise perceptions at discharge from inpatient spinal cord injury rehabilitation. Arch Phys Med Rehabil 2013, doi:10.1016/j.apmr.2013.05.011

19. Borg G. Psychophysical bases of perceived exertion. Med Sci Sports Exerc 1982;14:377-81.

20. Pickett GE, Campos-Benitez M, Keller JL, Duggal N. Epidemiology of traumatic spinal cord injury in Canada. Spine 2006;31:799-805.

21. Jacobs PL. Effects of resistance and endurance training in persons with paraplegia. Med Sci Sports Exerc 2009;41:992-7.

22. Ditor DS, Latimer AE, Martin Ginis KA, Arbour KP, McCartney N, Hicks AL. Maintenance of exercise participation in individuals with spinal cord injury: effects on quality of life, stress and pain. Spinal Cord 2003;41:446-50. 
PhD Thesis - Chelsea A. Pelletier - McMaster University - Kinesiology

23. Rimmer JH, Riley B, Wang E, Rauworth A, Jurkowski J. Physical activity participation among persons with disabilities: barriers and facilitators. Am J Prev Med 2004;26:419-25.

24. Cowan RE, Nash MS, Anderson KD. Exercise participation barrier prevalence and association with exercise participation status in individuals with spinal cord injury. Spinal Cord 2013;51:27-32.

25. Scelza WM, Kalpakjian CZ, Zemper ED, Tate DG. Perceived barriers to exercise in people with spinal cord injury. Am J Phys Med Rehabil 2005;84:576-83. 
PhD Thesis - Chelsea A. Pelletier - McMaster University - Kinesiology

Table 1. Participant Characteristics

\begin{tabular}{lcc}
\hline Characteristic & PAG & CON \\
\hline Sample size $(n)$ & 8 & 8 \\
Age (years) & $40.0 \pm 12.3(25-56)$ & $45.9 \pm 11.5(26-65)$ \\
Sex (male/female) & $8 / 0$ & $7 / 1$ \\
BMI $\left(\mathrm{kg} / \mathrm{m}^{2}\right)$ & $27.8 \pm 6.28(17.2-35.1)$ & $25.6 \pm 4.74(19.1-31.1)$ \\
Level of Injury & C $3-\mathrm{T} 10$ & $\mathrm{C} 1-\mathrm{T} 11$ \\
Time Post Injury & $15.0 \pm 8.52(1-28)$ & $9.25 \pm 10.0(2-31)$ \\
AIS & A: 2, B: 2, C: 3, D: 1 & A: 4, B: 0, C: 3, D: 1 \\
\hline
\end{tabular}

Values are mean \pm SD (range). Abbreviations: AIS, American Spinal Injury Association Impairment Score; BMI, body mass index 
PhD Thesis - Chelsea A. Pelletier - McMaster University - Kinesiology
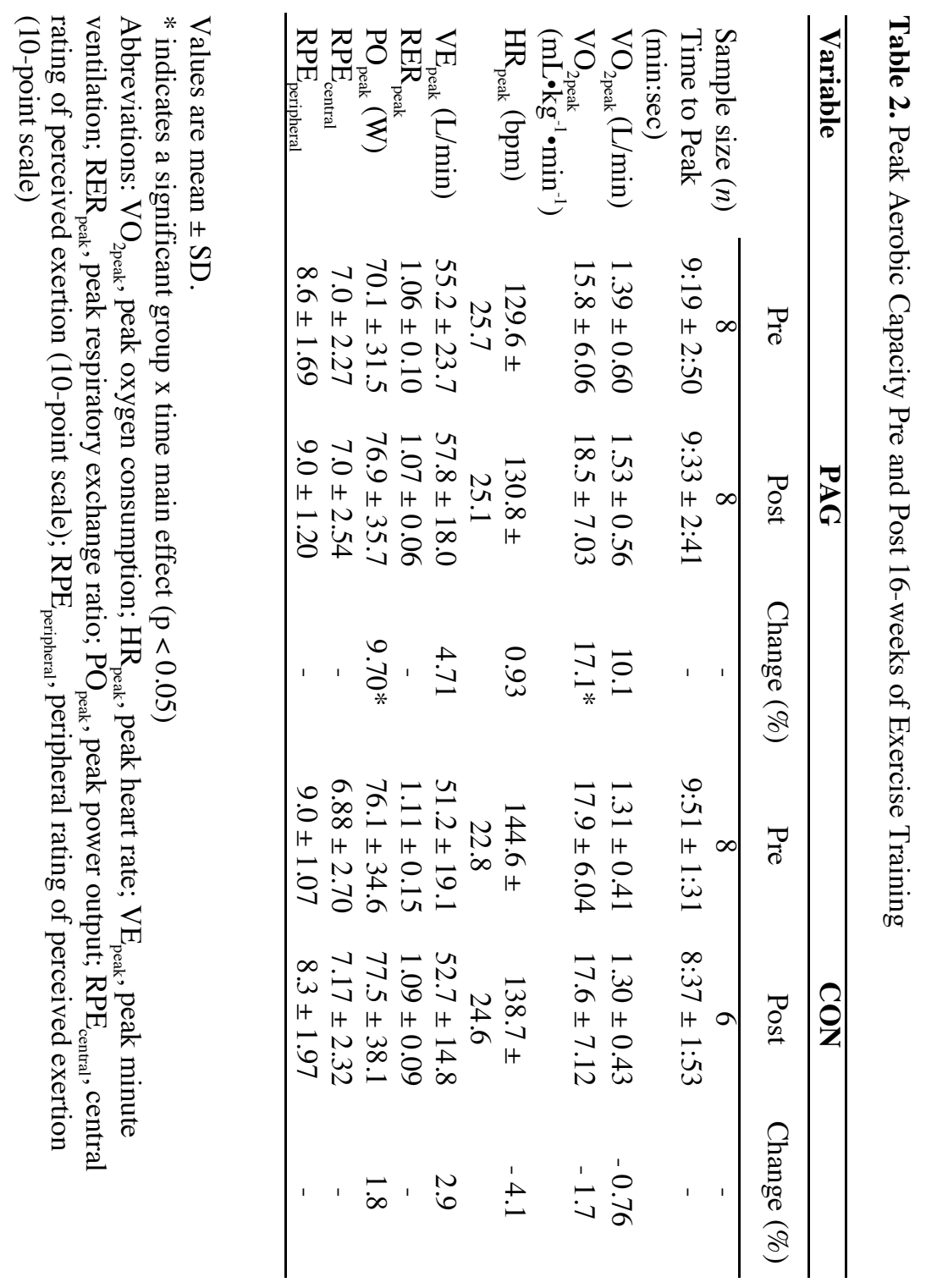
PhD Thesis - Chelsea A. Pelletier - McMaster University - Kinesiology

Table 3. Aerobic Endurance Pre and Post 16-weeks of Exercise Training

\begin{tabular}{lcccc}
\hline Variable & \multicolumn{2}{c}{ PAG } & \multicolumn{2}{c}{ CON } \\
\hline & Pre & Post & Pre & Post \\
\cline { 2 - 5 } Sample size $(n)$ & 7 & 7 & 7 & 6 \\
Power Output $(\mathrm{W})$ & $57.1 \pm 22.2$ & $64.3 \pm 21.7^{*}$ & $51.4 \pm 21.7$ & $51.7 \pm 25.0$ \\
$\begin{array}{l}\text { Performance Time } \\
(\text { min:sec) }\end{array}$ & $15: 18 \pm 4: 45$ & $17: 25 \pm 3: 33$ & $14: 59 \pm 5: 53$ & $16: 22 \pm 5: 10$ \\
$\begin{array}{l}\text { Steady state HR } \\
\text { (bpm) }\end{array}$ & $126.3 \pm 25.7$ & $130.3 \pm 27.8$ & $132.0 \pm 22.5$ & $129.7 \pm 24.3$ \\
$\begin{array}{l}\text { Steady state } \mathrm{VO}_{2} \\
\left(\mathrm{~mL}^{-1} \mathrm{~kg}^{-1} \cdot \mathrm{min}^{-1}\right)\end{array}$ & $14.8 \pm 5.65$ & $17.8 \pm 6.95$ & $14.8 \pm 7.8$ & $17.7 \pm 6.87$ \\
$\mathrm{RPE}_{\text {central }}$ & $6.7 \pm 1.38$ & $6.4 \pm 2.07$ & $7.3 \pm 1.98$ & $6.7 \pm 1.97$ \\
$\mathrm{RPE}_{\text {peripheral }}$ & $7.9 \pm 1.21$ & $8.0 \pm 1.41$ & $8.4 \pm 1.81$ & $7.8 \pm 2.1$ \\
\hline
\end{tabular}

Values are mean $\pm \mathrm{SD}$.

* indicates a significant $(\mathrm{p}<0.05)$ group $\mathrm{x}$ time interaction

Abbreviations: $\mathrm{HR}$, heart rate; $\mathrm{VO}_{2}$, oxygen consumption; $\mathrm{RPE}_{\text {central }}$, central rating of perceived exertion (10-point scale); $\mathrm{RPE}_{\text {peripheral }}$, peripheral rating of perceived exertion (10-point scale) 
PhD Thesis - Chelsea A. Pelletier - McMaster University - Kinesiology

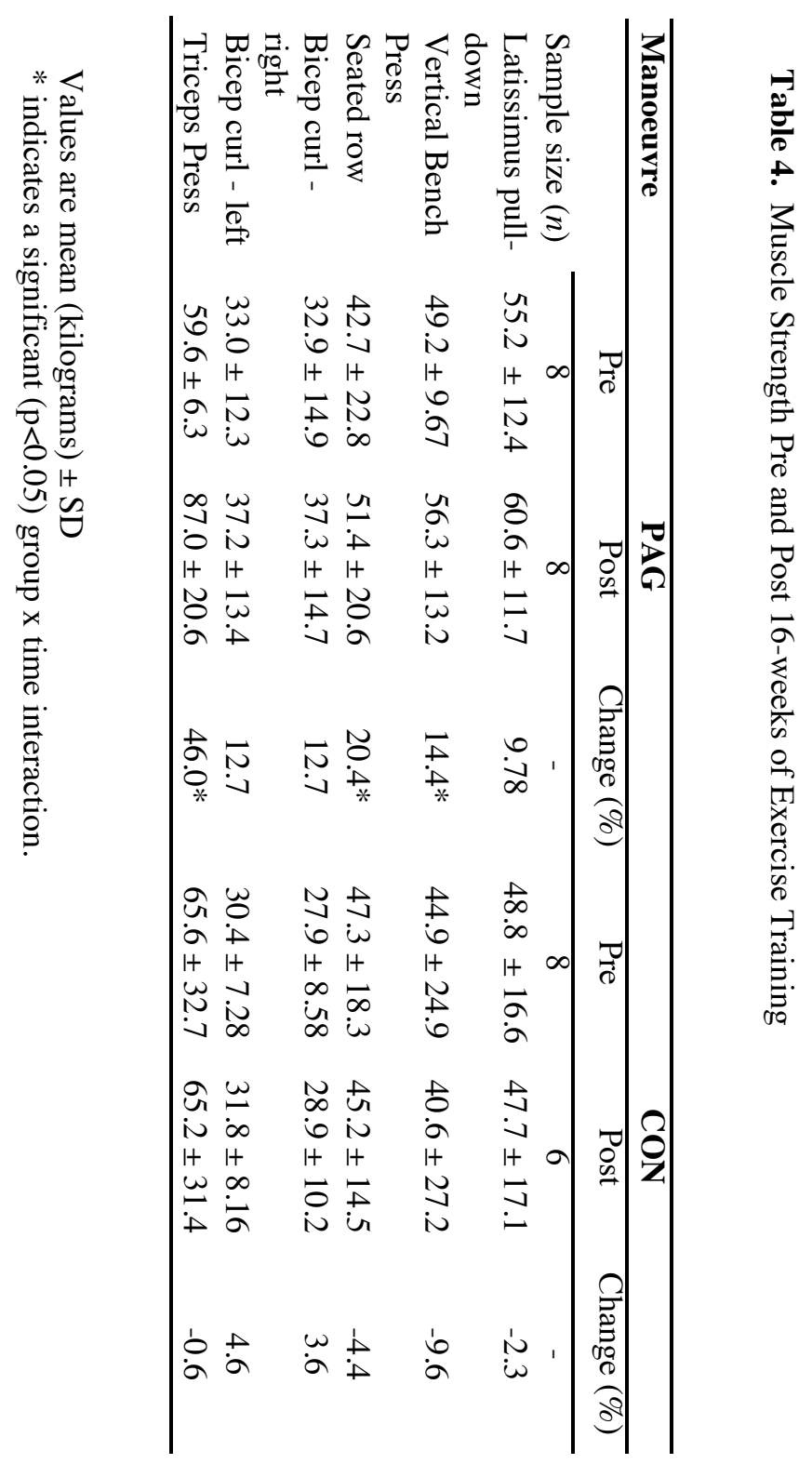




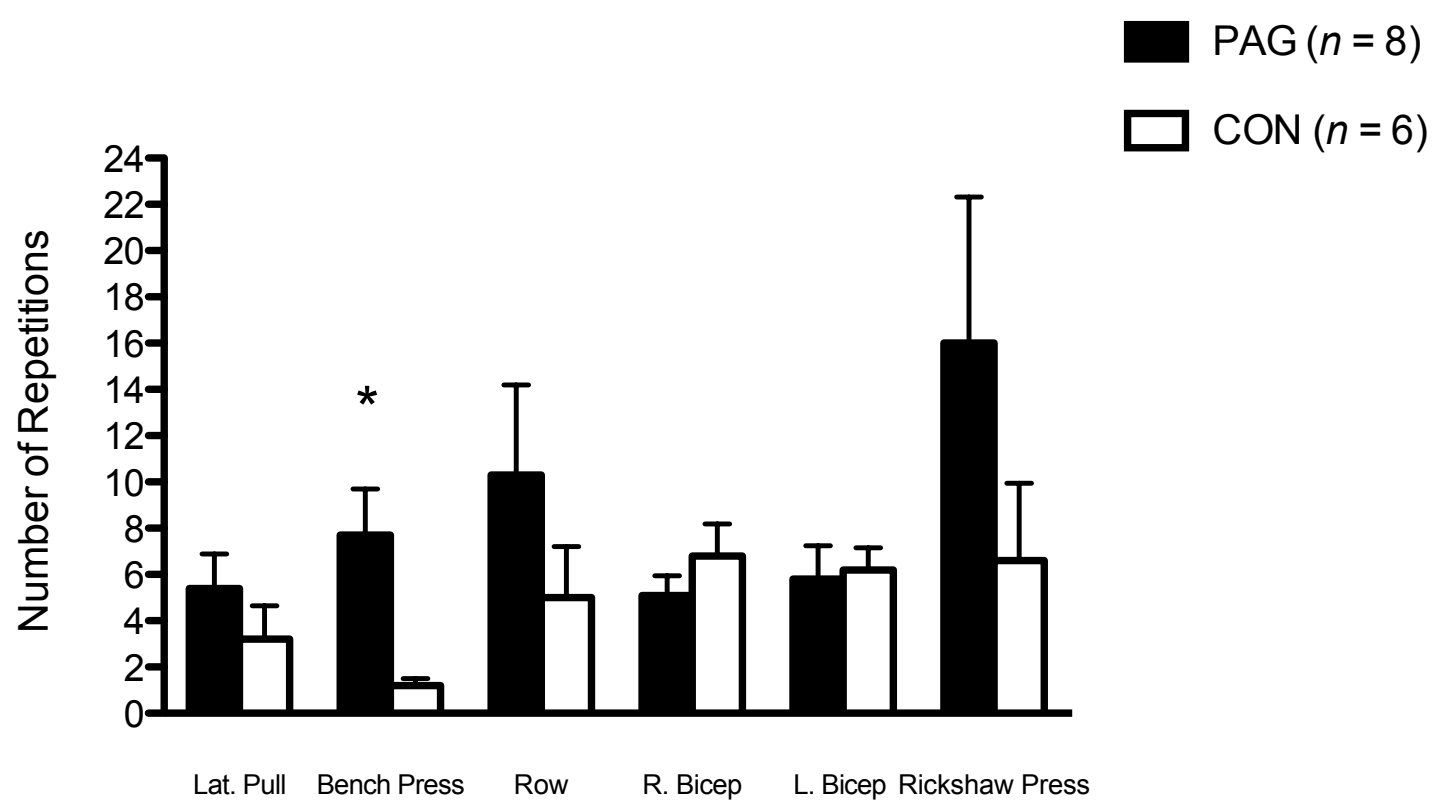

Figure 1. Muscle Endurance Following 16-weeks of Exercise Training

* indicates a significant difference $(\mathrm{p}<0.05)$ between PAG and CON groups 
PhD Thesis - Chelsea A. Pelletier - McMaster University - Kinesiology

Chapter 6

Discussion and Conclusions 
The purpose of this dissertation was to evaluate the role of exercise in the rehabilitation process after spinal cord injury (SCI). Taken together, this series of studies presents a potential model for integrating exercise into the continuity of care from inpatient rehabilitation through to discharge into the community. The importance of physical activity to the SCI population is becoming more and more evident as the growing body of literature is revealing important links between physical activity, cardiovascular health, ${ }^{1}$ metabolic disease, ${ }^{2}$ and functional ability. ${ }^{3,4}$ The results from this dissertation have confirmed the effectiveness of the physical activity guidelines for adults with SCI and have provided new information on how to optimize the exercise prescription for adults living in the community.

There is a negative health trajectory following rehabilitation ${ }^{5}$ and it is also a time characterised by a decrease in physical activity ${ }^{6}$ and an increase in body mass index. ${ }^{7}$ This decrease in health and function can theoretically be influenced by engagement in exercise at a suitable intensity, ${ }^{5,8}$ however, there are very few studies that have explored potential interventions at this stage. Further, decreasing length of stays in rehabilitation has resulted in a shift towards a reliance on outpatient services, despite a considerable lack of community infrastructure in place to support access to these services and/or engagement in exercise. ${ }^{9,10}$ This dissertation takes the first steps to fill some of the substantial gaps in knowledge surrounding the transitional phase after formal SCI rehabilitation is complete. One of the novel aspects of this dissertation is the combination of both physiological and psychosocial aspects related to exercise behaviour and prescription with the goal of 
encouraging the development of comprehensive interventions and health promotion strategies.

The purpose of the first study was to describe the aerobic capacity, orthostatic tolerance, and exercise perceptions among individuals prior to being discharged from inpatient SCI rehabilitation. This was an important first step to describe the overall readiness for exercise among individuals in the sub-acute phase of injury, and to begin identifying the focus for exercise interventions. The main findings of this study confirmed that peak oxygen consumption $\left(\mathrm{VO}_{2 \text { peak }}\right)$, power output $\left(\mathrm{PO}_{\text {peak }}\right)$, and heart rate were significantly lower among individuals with sub-acute tetraplegia compared to paraplegia. There was also a significant group difference in the response to the sit-up test, with $73 \%$ of participants with complete tetraplegia displaying a drop in blood pressure indicative of orthostatic hypotension compared with $25 \%$ and $0 \%$ of those with high or low complete paraplegia, respectively. The results of the exercise perceptions questionnaire revealed that participants at this stage of rehabilitation value exercise and see benefits to participation, however, they are generally not confident in their abilities to complete the task of either aerobic or resistance exercise training. Thus, while there is a certain amount of cardiovascular detraining and autonomic dysfunction that occurs secondary to SCI, ${ }^{11,12}$ it should not interfere with exercise participation. This is important new information that should be used to begin incorporating exercise testing and structured exercise training in the early phases of rehabilitation. 
Recently, a model of referral and cooperation between health care professionals and exercise specialists has been proposed during the transition from rehabilitation into the community, ${ }^{13}$ as educational programs have been shown to increase community reintegration post-discharge. ${ }^{14}$ The purpose of the second study was to evaluate the efficacy and feasibility of direct referral from rehabilitation to regular leisure time physical activity in accordance with the physical activity guidelines for adults with $\mathrm{SCI}^{15}$ combined with 16-weeks of telephone counselling support. The findings of this study support the efficacy of the system of direct referral and follow-up counselling, with adherence rates of $71 \%$ among those discharged from inpatient and $50 \%$ among those discharged from outpatient rehabilitation programs. While the efficacy of similar interventions have been demonstrated in the cardiac rehabilitation model, ${ }^{16}$ this was the first to explore its potential application to the rehabilitation of the SCI population. These findings offer preliminary support of direct referral and continued support for the first few months post-discharge, and conceivably could also be applied to other long-term rehabilitation outcomes (ie. self-care).

Once individuals are considered to have a chronic SCI ( $>1$ year post injury), there are now specific physical activity guidelines that describe the amount of exercise needed to improve aspects of fitness. ${ }^{15,17}$ These guidelines, however, have yet to be evaluated within a community setting. The final two studies of this dissertation focused on individuals with chronic SCI living in the community with the goal of both validating the guidelines and refining the exercise prescription process for these individuals. The purpose of the 
first study was to identify the optimal mode of aerobic and resistance exercise in terms of cardiovascular demand, energy expenditure, and consumer preference. The results of this study reveal that while no piece of exercise equipment was specifically preferred, the arm-only aerobic exercise modes were perceived as safer than hybrid (arm and leg) aerobic exercise modes. Safety was also rated as significantly higher among participants with paraplegia compared to those with tetraplegia for all aerobic and resistance exercise equipment types. There was no cardiovascular or metabolic advantage to using arm-only versus hybrid aerobic exercise types, suggesting that arm-only exercise may be the most appropriate exercise modality especially among community programs with limited resources. This study also confirmed the appropriateness of using ratings of perceived exertion to achieve a desired exercise intensity in people with SCI.

The final study in this dissertation validated the physical activity guidelines for adults with SCI in terms of improving aspects of physical fitness in a community sample. This is one of few randomized controlled exercise training studies in this population, and the first to evaluate the guidelines specifically. The results of this study revealed significant increases in aerobic capacity $\left(\mathrm{VO}_{2 \text { peak }}, \mathrm{PO}_{\text {peak }}\right.$, submaximal $\left.\mathrm{PO}\right)$, muscle strength (vertical bench press, seated row, rickshaw press), and muscle endurance (vertical bench press) compared to an active control group. Adherence rates were also excellent in the training group $(87.9 \pm 7.9 \%)$ and statistically significant compared to the active controls $(48.7 \pm$ $34.4 \%)$. 
By removing some of the most commonly reported barriers to exercise participation (ie. resource and facility accessibility, knowledge, cost), ${ }^{18,19}$ these studies have demonstrated strong adherence rates to the physical activity guidelines both within the community and at the point immediately following discharge from rehabilitation. Future investigations should now be directed at designing longitudinal interventions to encourage sustained participation in exercise programs (beyond the 4-month period). Improved cooperation between health care professionals and exercise specialists at outpatient or community programs may be a promising means by which to promote exercise as individuals reintegrate into the community, and identifies a unique role for a Kinesiologist in the health care model.

The physical activity guidelines for adults with SCI appear to be effective in improving measures of physical fitness among people living in the community. While there is currently insufficient evidence in the acute SCI population to develop a separate set of guidelines, the results of this series of studies indicate no reason why the guidelines cannot be applied to individuals who are more recently injured. From our testing, mean values for $\mathrm{VO}_{2 \text { peak }}$ do not appear to vary significantly between adults with sub-acute (14.5 $\left.\mathrm{mL} \cdot \mathrm{kg}^{-1} \cdot \mathrm{min}^{-1}\right)$ and chronic $\left(15.8 \mathrm{~mL} \cdot \mathrm{kg}^{-1} \cdot \mathrm{min}^{-1}\right) \mathrm{SCI}$, although there are larger differences between $\mathrm{PO}_{\text {peak }}$ (sub-acute: 49.2 W; chronic: 70.1 W). These comparisons suggest that changes in exercise capacity post-rehabilitation are most likely due to increases in muscle strength rather than central changes in cardiovascular efficiency or 
oxygen utilization. Therefore, for example, the exercise prescription for the sub-acute SCI population may be modified slightly to increase the focus on resistance training.

Identifying a means to accurately prescribe exercise intensity, especially among persons with tetraplegia, remains as an important research question. Findings from the present investigation support the use of ratings of perceived exertion (RPE) as a practical means to achieve a desired intensity, although splitting the prescription into central and peripheral components may important to ensure participants are exercising at a high enough cardiovascular intensity. ${ }^{21}$

In conclusion, this dissertation has presented a process to incorporate physical activity throughout the rehabilitation stages after SCI. It has been demonstrated that people with SCI are physiologically ready to participate in exercise at discharge from inpatient rehabilitation, but work is still needed to improve their confidence in their ability to perform exercise. The data show that direct referral to community exercise (from a healthcare provider) is well received by newly injured individuals, but that an initial period of counselling support may help participants adhere with their community exercise program. Finally, it's been shown that the physical activity guidelines are easily implemented within a community setting, and that they are effective in improving aspects of fitness. 


\section{References}

1. de Groot S, Post MW, Snoek GJ, Schuitemaker M, van der Woude LH. Longitudinal association between lifestyle and coronary heart disease risk factors among individuals with spinal cord injury. Spinal Cord 2012;51:314-8.

2. Koury JC, Passos MCF, Figueiredo FA, Chain A, Franco JG. Time of physical exercise practice after injury in cervical spinal cord-injured men is related to the increase in insulin sensitivity. Spinal Cord 2012;

3. Noreau L, Shephard RJ, Simard C, Paré G, Pomerleau P. Relationship of impairment and functional ability to habitual activity and fitness following spinal cord injury. Int J Rehabil Res 1993;16:265-75.

4. Dallmeijer AJ, van der Woude LHV. Health related functional status in men with spinal cord injury: relationship with lesion level and endurance capacity. Spinal Cord 2001;39:577-83.

5. Rimmer JH. Getting Beyond the Plateau: Bridging the gap between rehabilitation and community-based exercise. PMRJ 2012;4:857-61.

6. van den Berg-Emons RJ, Bussmann JB, Haisma JA, Sluis TA, van der Woude LH, Bergen MP, et al. A prospective study on physical activity levels after spinal cord injury during inpatient rehabilitation and the year after discharge. Arch Phys Med Rehabil 2008;89:2094-101.

7. de Groot S, Post M, Postma K, Sluis TA, van der Woude L. Prospective analysis of body mass index during and up to 5 years after discharge from inpatient spinal cord injury rehabilitation. J Rehabil Med 2010;42:922-8.

8. Nooijen CFJ, de Groot S, Postma K, Bergen MP, Stam HJ, Bussmann JBJ, et al. A more active lifestyle in persons with a recent spinal cord injury benefits physical fitness and health. Spinal Cord 2011;50:320-3.

9. Whiteneck GG, Gassaway J, Dijkers MP, Lammertse DP, Hammond F, Heinemann AW, et al. Inpatient and postdischarge rehabilitation services provided in the first year after spinal cord injury: findings from the SCIRehab study. Arch Phys Med Rehabil 2011;92:361-8.

10. Craven C, Verrier M, Balioussis C, Wolfe DL, J H, Noonan V, et al. Capturing capacity in Canadian SCI rehabilitation. Rick Hansen Institute; 2012. 
PhD Thesis - Chelsea A. Pelletier - McMaster University - Kinesiology

11. Van Loan MD, McCluer S, Loftin JM, Boileau RA. Comparison of physiological responses to maximal arm exercise among able-bodied, paraplegics and quadriplegics. Paraplegia 1987;25:397-405.

12. Claydon VE, Krassioukov AV. Orthostatic hypotension and autonomic pathways after spinal cord injury. J Neurotraum 2006;23:1713-25.

13. Rimmer JH, Henley KY. Building the crossroad between inpatient/outpatient rehabilitation and lifelong community-based fitness for people with neurologic disability. J Neurol Phys Ther 2013;37:72-7.

14. Tate DG, Forchheimer M. Enhancing community reintegration after inpatient rehabilitation for persons with spinal cord injury. Top Spinal Cord Inj Rehabil 1998;4:42-55.

15. Ginis KAM, Hicks AL, Latimer AE, Warburton DER, Bourne C, Ditor DS, et al. The development of evidence-informed physical activity guidelines for adults with spinal cord injury. Spinal Cord 2011;49:1088-96.

16. Sniehotta FF, Scholz U, Schwarzer R. Action plans and coping plans for physical exercise: a longitudinal intervention study in cardiac rehabilitation. Brit J Health Psychol 2010;11:23-37.

17. Hicks AL, Martin Ginis KA, Pelletier CA, Ditor DS, Foulon B, Wolfe DL. The effects of exercise training on physical capacity, strength, body composition and functional performance among adults with spinal cord injury: a systematic review. Spinal Cord 2011;49:1103-27.

18. Rimmer JH, Riley B, Wang E, Rauworth A, Jurkowski J. Physical activity participation among persons with disabilities: barriers and facilitators. Am J Prev Med 2004;26:419-25.

19. Cowan RE, Nash MS, Anderson KD. Exercise participation barrier prevalence and association with exercise participation status in individuals with spinal cord injury. Spinal Cord 2013;51:27-32.

20. Sisto SA, Lorenz DJ, Hutchinson K, Wenzel L, Harkema SJ, Krassioukov A. Cardiovascular status of individuals with incomplete spinal cord injury from 7 NeuroRecovery Network rehabilitation centers. Arch Phys Med Rehabil 2012;93:1578-87.

21. Cowan RE, Ginnity KL, Kressler J, Nash MS. Assessment of the talk test and rating of perceived exertion for exercise intensity prescription in persons with paraplegia. Top Spinal Cord Inj Rehabil 2012;18:212-9. 
PhD Thesis - Chelsea A. Pelletier - McMaster University - Kinesiology

\section{Appendices}




\section{A. Chapter 2}

\section{A.1 Exercise Self-Efficacy Questionnaire}

\section{Self-efficacy for Exercise Post-Discharge}

Instructions

We are interested in your opinions about engaging in leisure time physical activity after you have been discharged from rehabilitation. Please use the following definition of regular leisure time physical activity as you respond to these questions:

- Leisure Time Physical Activity: All of the activities that you choose to do during your free time that requires physical exertion (e.g., exercise, sports). To be considered "regular" these activities should be done for at least 30 minutes a day most days of the week. The 30 minutes may be accumulated throughout the day.

Outcome value

\begin{tabular}{|c|c|c|c|c|c|c|c|}
\hline \multirow[b]{2}{*}{$\begin{array}{l}\text { In the next three months post-discharge, engaging in regular } \\
\text { leisure time physical activity is the most important thing I can do }\end{array}$} & \multicolumn{5}{|c|}{$\begin{array}{l}\text { Strongly } \\
\text { Disagree }\end{array}$} & \multicolumn{2}{|c|}{$\begin{array}{r}\text { Strongly } \\
\text { Agree }\end{array}$} \\
\hline & 1 & 2 & 3 & 4 & 5 & 6 & 7 \\
\hline $\begin{array}{l}\text { Establishing a routine including regular leisure time physical } \\
\text { activity in the next three months post-discharge is a high priority } \\
\text { for me }\end{array}$ & 1 & 2 & 3 & 4 & 5 & 6 & 7 \\
\hline $\begin{array}{l}\text { Engaging in regular leisure time physical activity post-discharge } \\
\text { would be extremely valuable }\end{array}$ & 1 & 2 & 3 & 4 & 5 & 6 & 7 \\
\hline
\end{tabular}

Outcome expectations

I think that engaging regular leisure time physical activity in the next three months post-discharge will...

\begin{tabular}{|c|c|c|c|c|c|c|c|}
\hline \multirow[b]{2}{*}{ increase my mobility } & \multicolumn{5}{|c|}{$\begin{array}{l}\text { Strongly } \\
\text { Disagree }\end{array}$} & \multicolumn{2}{|c|}{$\begin{array}{r}\text { Strongly } \\
\text { Agree }\end{array}$} \\
\hline & 1 & 2 & 3 & 4 & 5 & 6 & 7 \\
\hline put me at risk of experiencing autonomic dysreflexia & 1 & 2 & 3 & 4 & 5 & 6 & 7 \\
\hline interfere with my healing & 1 & 2 & 3 & 4 & 5 & 6 & 7 \\
\hline increase the pain I am experiencing & 1 & 2 & 3 & 4 & 5 & 6 & 7 \\
\hline $\begin{array}{l}\text { make my activities of daily living (e.g. getting dressed, housework) } \\
\text { easier to perform }\end{array}$ & 1 & 2 & 3 & 4 & 5 & 6 & 7 \\
\hline increase my energy level & 1 & 2 & 3 & 4 & 5 & 6 & 7 \\
\hline $\begin{array}{l}\text { increase my confidence to do the things I need or want to do } \\
\text { (e.g. transferring in and out of your chair) }\end{array}$ & 1 & 2 & 3 & 4 & 5 & 6 & 7 \\
\hline prevent secondary health conditions (e.g. pressure sores) & 1 & 2 & 3 & 4 & 5 & 6 & 7 \\
\hline increase my self-esteem & 1 & 2 & 3 & 4 & 5 & 6 & 7 \\
\hline increase my confidence in social situations & 1 & 2 & 3 & 4 & 5 & 6 & 7 \\
\hline be very risky & 1 & 2 & 3 & 4 & 5 & 6 & 7 \\
\hline be very dangerous & 1 & 2 & 3 & 4 & 5 & 6 & 7 \\
\hline
\end{tabular}


$\underline{\text { Self-efficacy beliefs }}$

Assuming that you are very motivated, in the next three months post-discharge, how confident are you that you can fit 30 min of leisure time physical activity into your weekly schedule:

\begin{tabular}{llllllll}
\hline & \multicolumn{1}{c}{$\begin{array}{c}\text { Not at all } \\
\text { confident }\end{array}$} & & \multicolumn{2}{c}{$\begin{array}{r}\text { Completely } \\
\text { confident }\end{array}$} \\
\hline once per week & 1 & 2 & 3 & 4 & 5 & 6 & 7 \\
twice per week & 1 & 2 & 3 & 4 & 5 & 6 & 7 \\
three times per week & 1 & 2 & 3 & 4 & 5 & 6 & 7 \\
more than three times per week & 1 & 2 & 3 & 4 & 5 & 6 & 7 \\
\hline
\end{tabular}

In the next three months post-discharge, how confident are you that you can:

\begin{tabular}{|c|c|c|c|c|c|c|c|}
\hline \multirow[b]{2}{*}{$\begin{array}{l}\text { Set realistic goals for engaging in regular leisure time } \\
\text { physical activity }\end{array}$} & \multicolumn{4}{|c|}{$\begin{array}{l}\text { Not at all } \\
\text { confident }\end{array}$} & \multicolumn{3}{|c|}{$\begin{array}{r}\text { Completely } \\
\text { confident }\end{array}$} \\
\hline & 1 & 2 & 3 & 4 & 5 & 6 & 7 \\
\hline $\begin{array}{l}\text { Develop a plan for reaching your leisure time physical } \\
\text { activity goals }\end{array}$ & 1 & 2 & 3 & 4 & 5 & 6 & 7 \\
\hline $\begin{array}{l}\text { Coordinate the resources I will need to engage in } \\
\text { regular leisure time physical activity within my own } \\
\text { home }\end{array}$ & 1 & 2 & 3 & 4 & 5 & 6 & 7 \\
\hline $\begin{array}{l}\text { Coordinate the resources I will need to engage in } \\
\text { regular leisure time physical activity within my } \\
\text { community }\end{array}$ & 1 & 2 & 3 & 4 & 5 & 6 & 7 \\
\hline
\end{tabular}

The next few questions ask about AEROBIC leisure time physical activity. This includes activities that typically increase heart rate and breathing such as wheeling, cycling on an arm ergometer, swimming, and basketball.

Considering how you feel right now, how confident are you that you could physically do the following amounts of MODERATE intensity AEROBIC activity without stopping.

\begin{tabular}{lllllllcc}
\hline & \multicolumn{2}{c}{$\begin{array}{c}\text { Not at all } \\
\text { confident }\end{array}$} & \multicolumn{4}{c}{$\begin{array}{c}\text { Completely } \\
\text { confident }\end{array}$} \\
\hline $\mathbf{1 0}$ min & 1 & 2 & 3 & 4 & 5 & 6 & 7 \\
$\mathbf{2 0}$ min & 1 & 2 & 3 & 4 & 5 & 6 & 7 \\
$\mathbf{3 0}$ min & 1 & 2 & 3 & 4 & 5 & 6 & 7 \\
$\mathbf{4 5} \mathbf{~ m i n}$ & 1 & 2 & 3 & 4 & 5 & 6 & 7 \\
$\mathbf{6 0} \mathbf{~ m i n}$ & 1 & 2 & 3 & 4 & 5 & 6 & 7 \\
\hline
\end{tabular}


Considering how you feel right now, how confident are you that you could physically do the following amounts of HEAVY intensity AEROBIC activity without stopping.

\begin{tabular}{llllllllc}
\hline & \multicolumn{2}{c}{$\begin{array}{c}\text { Not at all } \\
\text { confident }\end{array}$} & & \multicolumn{3}{c}{$\begin{array}{c}\text { Completely } \\
\text { confident }\end{array}$} \\
\hline $\mathbf{1 0}$ min & 1 & 2 & 3 & 4 & 5 & 6 & 7 \\
$\mathbf{2 0}$ min & 1 & 2 & 3 & 4 & 5 & 6 & 7 \\
$\mathbf{3 0} \mathbf{~ m i n}$ & 1 & 2 & 3 & 4 & 5 & 6 & 7 \\
$\mathbf{4 5}$ min & 1 & 2 & 3 & 4 & 5 & 6 & 7 \\
$\mathbf{6 0} \mathbf{m i n}$ & 1 & 2 & 3 & 4 & 5 & 6 & 7 \\
\hline
\end{tabular}

How confident are you that you can...

\begin{tabular}{|c|c|c|c|c|c|c|c|}
\hline \multirow[b]{2}{*}{ Perform aerobic fitness exercises correctly } & \multicolumn{4}{|c|}{$\begin{array}{l}\text { Not at all } \\
\text { confident }\end{array}$} & \multicolumn{3}{|c|}{$\begin{array}{r}\text { Completely } \\
\text { confident }\end{array}$} \\
\hline & 1 & 2 & $\overline{3}$ & 2 & 5 & 6 & 7 \\
\hline $\begin{array}{l}\text { Adapt aerobic fitness exercises to accommodate your } \\
\text { ability }\end{array}$ & 1 & 2 & 3 & 2 & 5 & 6 & 7 \\
\hline $\begin{array}{l}\text { Design an aerobic training program to achieve your } \\
\text { aerobic fitness goals }\end{array}$ & 1 & 2 & 3 & 2 & 5 & 6 & 7 \\
\hline Engage in aerobic fitness activities at home safely & 1 & 2 & 3 & 2 & 5 & 6 & 7 \\
\hline $\begin{array}{l}\text { Recognize when you need assistance with an aerobic } \\
\text { fitness activity }\end{array}$ & 1 & 2 & 3 & 2 & 5 & 6 & 7 \\
\hline
\end{tabular}

The next few questions ask about STRENGTHENING activities. These are activities that work your muscles, such as lifting weights and using exercise bands.

Considering how you feel right now, how confident are you that you could physically do the following amounts of MODERATE intensity STRENGTHENING activity without stopping.

\begin{tabular}{lllllllcc}
\hline & \multicolumn{3}{c}{$\begin{array}{c}\text { Not at all } \\
\text { confident }\end{array}$} & & \multicolumn{3}{c}{$\begin{array}{c}\text { Completely } \\
\text { confident }\end{array}$} \\
\hline $\mathbf{1 0}$ min & 1 & 2 & 3 & 4 & 5 & 6 & 7 \\
$\mathbf{2 0}$ min & 1 & 2 & 3 & 4 & 5 & 6 & 7 \\
$\mathbf{3 0} \mathbf{~ m i n}$ & 1 & 2 & 3 & 4 & 5 & 6 & 7 \\
$\mathbf{4 5} \mathbf{~ m i n}$ & 1 & 2 & 3 & 4 & 5 & 6 & 7 \\
$\mathbf{6 0} \mathbf{~ m i n}$ & 1 & 2 & 3 & 4 & 5 & 6 & 7 \\
\hline
\end{tabular}


Considering how you feel right now, how confident are you that you could physically do the following amounts of HEAVY intensity STRENGTHENING activity without stopping.

\begin{tabular}{llllllll}
\hline & \multicolumn{2}{c}{$\begin{array}{c}\text { Not at all } \\
\text { confident }\end{array}$} & \multicolumn{4}{c}{$\begin{array}{c}\text { Completely } \\
\text { confident }\end{array}$} \\
\hline $\mathbf{1 0}$ min & 1 & 2 & 3 & 4 & 5 & 6 & 7 \\
$\mathbf{2 0}$ min & 1 & 2 & 3 & 4 & 5 & 6 & 7 \\
$\mathbf{3 0} \mathbf{m i n}$ & 1 & 2 & 3 & 4 & 5 & 6 & 7 \\
$\mathbf{4 5} \mathbf{m i n}$ & 1 & 2 & 3 & 4 & 5 & 6 & 7 \\
$\mathbf{6 0} \mathbf{m i n}$ & 1 & 2 & 3 & 4 & 5 & 6 & 7 \\
\hline
\end{tabular}

How confident are you that you can...

\begin{tabular}{|c|c|c|c|c|c|c|c|}
\hline \multirow[b]{2}{*}{ Perform strengthening exercises correctly } & \multicolumn{4}{|c|}{$\begin{array}{l}\text { Not at all } \\
\text { confident }\end{array}$} & \multicolumn{3}{|c|}{$\begin{array}{r}\text { Completely } \\
\text { confident }\end{array}$} \\
\hline & 1 & 2 & . & $\overline{4}$ & 5 & 6 & 7 \\
\hline $\begin{array}{l}\text { Adapt strengthening exercises to accommodate your } \\
\text { ability }\end{array}$ & 1 & 2 & & 4 & 5 & 6 & 7 \\
\hline $\begin{array}{l}\text { Design a strengthening training program to achieve } \\
\text { your strength goals }\end{array}$ & 1 & 2 & & 4 & 5 & 6 & 7 \\
\hline Engage in strengthening activities at home safely & 1 & 2 & & 4 & 5 & 6 & 7 \\
\hline $\begin{array}{l}\text { Recognize when you need assistance with a } \\
\text { strengthening activity }\end{array}$ & 1 & 2 & & 4 & 5 & 6 & 7 \\
\hline
\end{tabular}




\section{B. Chapter 3}

\section{B.1 Exercise Beliefs Questionnaire}

\section{Self-efficacy for Exercise Post-Discharge}

Instructions

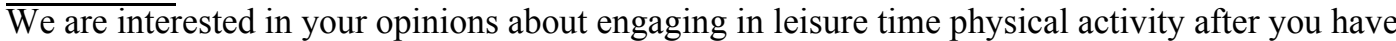
been discharged from rehabilitation. Please use the following definition of regular leisure time physical activity as you respond to these questions:

- Leisure Time Physical Activity: All of the activities that you choose to do during your free time that requires physical exertion (e.g., exercise, sports). To be considered "regular" these activities should be done for at least 30 minutes a day most days of the week. The 30 minutes may be accumulated throughout the day.

Outcome value

\begin{tabular}{|c|c|c|c|c|c|c|}
\hline & $\begin{array}{l}\text { Strongly } \\
\text { Disagree }\end{array}$ & & & & \multicolumn{2}{|c|}{$\begin{array}{r}\text { Strongly } \\
\text { Agree }\end{array}$} \\
\hline $\begin{array}{l}\text { In the next four months post-discharge, engaging in regular } \\
\text { leisure time physical activity is the most important thing I } \\
\text { can do }\end{array}$ & 1 & 3 & 4 & 5 & 6 & 7 \\
\hline $\begin{array}{l}\text { Establishing a routine including regular leisure time physical } \\
\text { activity in the next four months post-discharge is a high } \\
\text { priority for me }\end{array}$ & 1 & 3 & 4 & 5 & 6 & 7 \\
\hline $\begin{array}{l}\text { Engaging in regular leisure time physical activity post- } \\
\text { discharge would be extremely valuable }\end{array}$ & 1 & 3 & 4 & 5 & 6 & 7 \\
\hline
\end{tabular}

Outcome expectations

I think that engaging regular leisure time physical activity in the next four months post-discharge will...

\begin{tabular}{|c|c|c|c|c|c|c|c|}
\hline \multirow[b]{2}{*}{ increase my mobility } & \multicolumn{5}{|c|}{$\begin{array}{l}\text { Strongly } \\
\text { Disagree }\end{array}$} & \multicolumn{2}{|c|}{$\begin{array}{r}\text { Strongly } \\
\text { Agree } \\
\end{array}$} \\
\hline & 1 & 2 & 3 & 4 & 5 & 6 & 7 \\
\hline put me at risk of experiencing autonomic dysreflexia & 1 & 2 & 3 & 4 & 5 & 6 & 7 \\
\hline interfere with my healing & 1 & 2 & 3 & 4 & 5 & 6 & 7 \\
\hline increase the pain I am experiencing & 1 & 2 & 3 & 4 & 5 & 6 & 7 \\
\hline $\begin{array}{l}\text { make my activities of daily living (e.g. getting dressed, } \\
\text { housework) easier to perform }\end{array}$ & 1 & 2 & 3 & 4 & 5 & 6 & 7 \\
\hline increase my energy level & 1 & 2 & 3 & 4 & 5 & 6 & 7 \\
\hline $\begin{array}{l}\text { increase my confidence to do the things I need or want to do } \\
\text { (e.g. transferring in and out of your chair) }\end{array}$ & 1 & 2 & 3 & 4 & 5 & 6 & 7 \\
\hline prevent secondary health conditions (e.g. pressure sores) & 1 & 2 & 3 & 4 & 5 & 6 & 7 \\
\hline increase my self-esteem & 1 & 2 & 3 & 4 & 5 & 6 & 7 \\
\hline increase my confidence in social situations & 1 & 2 & 3 & 4 & 5 & 6 & 7 \\
\hline be very risky & 1 & 2 & 3 & 4 & 5 & 6 & 7 \\
\hline be very dangerous & 1 & 2 & 3 & 4 & 5 & 6 & 7 \\
\hline
\end{tabular}


Self-efficacy beliefs

Assuming that you are very motivated, in the next four months post-discharge, how confident are you that you can fit 30 min of leisure time physical activity into your weekly schedule:

\begin{tabular}{|c|c|c|c|c|c|c|c|}
\hline \multirow[b]{2}{*}{ once per week } & \multicolumn{4}{|c|}{$\begin{array}{l}\text { Not at all } \\
\text { confident }\end{array}$} & \multicolumn{3}{|c|}{$\begin{array}{r}\text { Completely } \\
\text { confident }\end{array}$} \\
\hline & 1 & 2 & 3 & 4 & 5 & 6 & 7 \\
\hline twice per week & 1 & 2 & 3 & 4 & 5 & 6 & 7 \\
\hline three times per week & 1 & 2 & 3 & 4 & 5 & 6 & 7 \\
\hline more than three times per week & 1 & 2 & 3 & 4 & 5 & 6 & 7 \\
\hline
\end{tabular}

In the next four months post-discharge, how confident are you that you can:

\begin{tabular}{|c|c|c|c|c|c|c|c|}
\hline \multirow[b]{2}{*}{$\begin{array}{l}\text { Set realistic goals for engaging in regular leisure time } \\
\text { physical activity }\end{array}$} & \multicolumn{4}{|c|}{$\begin{array}{l}\text { Not at all } \\
\text { confident }\end{array}$} & \multicolumn{3}{|c|}{$\begin{array}{r}\text { Completely } \\
\text { confident }\end{array}$} \\
\hline & 1 & 2 & 3 & 4 & 5 & 6 & 7 \\
\hline $\begin{array}{l}\text { Develop a plan for reaching your leisure time physical } \\
\text { activity goals }\end{array}$ & 1 & 2 & 3 & 4 & 5 & 6 & 7 \\
\hline $\begin{array}{l}\text { Coordinate the resources I will need to engage in } \\
\text { regular leisure time physical activity within my own } \\
\text { home }\end{array}$ & 1 & 2 & 3 & 4 & 5 & 6 & 7 \\
\hline $\begin{array}{l}\text { Coordinate the resources I will need to engage in } \\
\text { regular leisure time physical activity within my } \\
\text { community }\end{array}$ & 1 & 2 & 3 & 4 & 5 & 6 & 7 \\
\hline
\end{tabular}

The next few questions ask about AEROBIC leisure time physical activity. This includes activities that typically increase heart rate and breathing such as wheeling, cycling on an arm ergometer, swimming, and basketball.

Considering how you feel right now, how confident are you that you could physically do the following amounts of MODERATE intensity AEROBIC activity without stopping.

\begin{tabular}{|c|c|c|c|c|c|c|c|}
\hline \multirow[b]{2}{*}{$10 \mathrm{~min}$} & \multicolumn{4}{|c|}{$\begin{array}{l}\text { Not at all } \\
\text { confident }\end{array}$} & \multicolumn{3}{|c|}{$\begin{array}{r}\text { Completely } \\
\text { confident }\end{array}$} \\
\hline & 1 & 2 & 3 & 4 & 5 & 6 & 7 \\
\hline $20 \mathrm{~min}$ & 1 & 2 & 3 & 4 & 5 & 6 & 7 \\
\hline $30 \mathrm{~min}$ & 1 & 2 & 3 & 4 & 5 & 6 & 7 \\
\hline $45 \mathrm{~min}$ & 1 & 2 & 3 & 4 & 5 & 6 & 7 \\
\hline $60 \mathrm{~min}$ & 1 & 2 & 3 & 4 & 5 & 6 & 7 \\
\hline
\end{tabular}


PhD Thesis - Chelsea A. Pelletier - McMaster University - Kinesiology

Considering how you feel right now, how confident are you that you could physically do the following amounts of HEAVY intensity AEROBIC activity without stopping.

\begin{tabular}{llllllll}
\hline & \multicolumn{2}{c}{$\begin{array}{c}\text { Not at all } \\
\text { confident }\end{array}$} & \multicolumn{4}{c}{$\begin{array}{c}\text { Completely } \\
\text { confident }\end{array}$} \\
\hline $\mathbf{1 0}$ min & 1 & 2 & 3 & 4 & 5 & 6 & 7 \\
$\mathbf{2 0}$ min & 1 & 2 & 3 & 4 & 5 & 6 & 7 \\
$\mathbf{3 0}$ min & 1 & 2 & 3 & 4 & 5 & 6 & 7 \\
$\mathbf{4 5}$ min & 1 & 2 & 3 & 4 & 5 & 6 & 7 \\
$\mathbf{6 0}$ min & 1 & 2 & 3 & 4 & 5 & 6 & 7 \\
\hline
\end{tabular}

How confident are you that you can...

\begin{tabular}{|c|c|c|c|c|c|c|c|}
\hline \multirow[b]{2}{*}{ Perform aerobic fitness exercises correctly } & \multicolumn{4}{|c|}{$\begin{array}{l}\text { Not at all } \\
\text { confident }\end{array}$} & \multicolumn{3}{|c|}{$\begin{array}{r}\text { Completely } \\
\text { confident }\end{array}$} \\
\hline & 1 & 2 & 3 & 4 & 5 & 6 & 7 \\
\hline $\begin{array}{l}\text { Adapt aerobic fitness exercises to accommodate your } \\
\text { ability }\end{array}$ & 1 & 2 & 3 & 4 & 5 & 6 & 7 \\
\hline $\begin{array}{l}\text { Design an aerobic training program to achieve your } \\
\text { aerobic fitness goals }\end{array}$ & 1 & 2 & 3 & 4 & 5 & 6 & 7 \\
\hline Engage in aerobic fitness activities at home safely & 1 & 2 & 3 & 4 & 5 & 6 & 7 \\
\hline $\begin{array}{l}\text { Recognize when you need assistance with an aerobic } \\
\text { fitness activity }\end{array}$ & 1 & 2 & 3 & 4 & 5 & 6 & 7 \\
\hline
\end{tabular}

The next few questions ask about STRENGTHENING activities. These are activities that work your muscles, such as lifting weights and using exercise bands.

Considering how you feel right now, how confident are you that you could physically do the following amounts of MODERATE intensity STRENGTHENING activity without stopping.

\begin{tabular}{llllllll}
\hline & \multicolumn{2}{c}{$\begin{array}{c}\text { Not at all } \\
\text { confident }\end{array}$} & \multicolumn{4}{c}{$\begin{array}{r}\text { Completely } \\
\text { confident }\end{array}$} \\
\hline $\mathbf{1 0}$ min & 1 & 2 & 3 & 4 & 5 & 6 & 7 \\
$\mathbf{2 0}$ min & 1 & 2 & 3 & 4 & 5 & 6 & 7 \\
$\mathbf{3 0}$ min & 1 & 2 & 3 & 4 & 5 & 6 & 7 \\
$\mathbf{4 5}$ min & 1 & 2 & 3 & 4 & 5 & 6 & 7 \\
$\mathbf{6 0}$ min & 1 & 2 & 3 & 4 & 5 & 6 & 7 \\
\hline
\end{tabular}


PhD Thesis - Chelsea A. Pelletier - McMaster University - Kinesiology

Considering how you feel right now, how confident are you that you could physically do the following amounts of HEAVY intensity STRENGTHENING activity without stopping.

\begin{tabular}{lllllllcc}
\hline & \multicolumn{2}{c}{$\begin{array}{c}\text { Not at all } \\
\text { confident }\end{array}$} & \multicolumn{4}{c}{$\begin{array}{c}\text { Completely } \\
\text { confident }\end{array}$} \\
\hline $\mathbf{1 0}$ min & 1 & 2 & 3 & 4 & 5 & 6 & 7 \\
$\mathbf{2 0}$ min & 1 & 2 & 3 & 4 & 5 & 6 & 7 \\
$\mathbf{3 0}$ min & 1 & 2 & 3 & 4 & 5 & 6 & 7 \\
$\mathbf{4 5}$ min & 1 & 2 & 3 & 4 & 5 & 6 & 7 \\
$\mathbf{6 0}$ min & 1 & 2 & 3 & 4 & 5 & 6 & 7 \\
\hline
\end{tabular}

How confident are you that you can...

\begin{tabular}{|c|c|c|c|c|c|c|c|}
\hline \multirow[b]{2}{*}{ Perform strengthening exercises correctly } & \multicolumn{4}{|c|}{$\begin{array}{l}\text { Not at all } \\
\text { confident }\end{array}$} & \multicolumn{3}{|c|}{$\begin{array}{r}\text { Completely } \\
\text { confident }\end{array}$} \\
\hline & 1 & $\overline{2}$ & $\overline{3}$ & $\overline{4}$ & 5 & 6 & 7 \\
\hline $\begin{array}{l}\text { Adapt strengthening exercises to accommodate your } \\
\text { ability }\end{array}$ & 1 & 2 & 3 & 4 & 5 & 6 & 7 \\
\hline $\begin{array}{l}\text { Design a strengthening training program to achieve your } \\
\text { strength goals }\end{array}$ & 1 & 2 & 3 & 4 & 5 & 6 & 7 \\
\hline Engage in strengthening activities at home safely & 1 & 2 & 3 & 4 & 5 & 6 & 7 \\
\hline $\begin{array}{l}\text { Recognize when you need assistance with a strengthening } \\
\text { activity }\end{array}$ & 1 & 2 & 3 & 4 & 5 & 6 & 7 \\
\hline
\end{tabular}


PhD Thesis - Chelsea A. Pelletier - McMaster University - Kinesiology

\author{
B.2. Exercise Referral Form
}

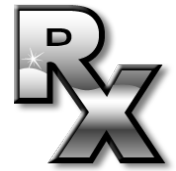

Exercise Referral Form

Patient's Name:

Date:

\begin{tabular}{l} 
HEALTH CARE PROFESSIONAL'S \\
RECOMMENDATION \\
Commit to improving your health by attending \\
MacWheelers twice a week. For maximum benefit, \\
be sure to perform the following activities during \\
each visit: \\
○ aerobic exercise \\
o strength exercise \\
o flexibility exercise \\
Signature: \\
\hline
\end{tabular}

For more information about MacWheelers contact the program coordinator, Susie Ward.

e-mail: bwstt@mcmaster.ca

phone: (905)525-9140 ext. 22576
Clearance for participation in physical activity

(To be completed by health care professional)

1. Spinal Cord Injury Level:

2. Functional Classification (ASIA score):

3. Date of Injury:

4. Medical Conditions that may affect exercise tolerance (ie Cardiovascular, metabolic, infection, lung, CNS, blood, musculoskeletal) :

5. Medications (indicate any exercise-related precautions):

6. Based on a current review of the health status of the above person, I recommend:
a Unrestricted Physical Activity
a Progressive Physical Activity,

with avoidance of

with inclusion of

Health care

professional's name:

Address:

Telephone:

Fax:

Signature: 


\section{Chapter 4}

\section{C.1 Consumer Preference Questionnaire}

\section{Equipment Evaluation}

Equipment name:

Equipment type (check all that apply):

$$
\begin{aligned}
& \text { Resistance } \\
& \text { Aerobic }
\end{aligned}
$$

Flexibility

Other:

Have you used this piece of equipment before? $\quad \bigcirc$ Yes $\quad \bigcirc$ No

If yes, how often do you use this piece of equipment?
$\bigcirc$ rarely
once or twice per month

$\bigcirc$ once per week

$\bigcirc$ twice per week

$\bigcirc$ more than two times per week

$\underline{\text { Evaluation of experience }}$

\begin{tabular}{|c|c|c|c|c|c|c|c|c|c|}
\hline $\begin{array}{c}-5 \\
\text { very } \\
\text { bad }\end{array}$ & -4 & $\begin{array}{c}-\mathbf{3} \\
\text { moderately } \\
\text { bad }\end{array}$ & -2 & $\begin{array}{c}-\mathbf{1} \\
\text { somewhat } \\
\text { bad }\end{array}$ & 0 & $\begin{array}{l}+1 \\
\text { somewhat } \\
\text { good }\end{array}$ & +2 & $\begin{array}{c}\mathbf{+ 3} \\
\text { moderately } \\
\text { Good }\end{array}$ & +4 \\
\hline
\end{tabular}

What number best represents how you feel right now?

Using the scale below, indicate the extent to which each word describes how you feel right now. Record your responses on the line next to each work.

\begin{tabular}{|c|c|c|c|}
\hline 1.Calm & 4. Energetic & 7. Tired & 10. Peaceful \\
\hline Fatigued & 5. Worn-out & 8. Нарpy & 11. Upbeat \\
\hline Refreshed & 6. Relaxed & 9. Enthusiastic & 12. Revived \\
\hline
\end{tabular}

\begin{tabular}{|llll|}
\hline $0=$ do not feel & $1=$ feel slightly $2=$ feel moderately & $3=$ feel strongly & $4=$ feel very strongly \\
\hline
\end{tabular}


PhD Thesis - Chelsea A. Pelletier - McMaster University - Kinesiology

During a typical exercise session...

\begin{tabular}{llllllll}
\hline & \multicolumn{2}{l}{$\begin{array}{l}\text { None at } \\
\text { all }\end{array}$} & & & \multicolumn{3}{c}{ A lot } \\
\hline How much shoulder pain do you usually experience & 1 & 2 & 3 & 4 & 5 & 6 & 7 \\
How much bodily pain do you usually experience & 1 & 2 & 3 & 4 & 5 & 6 & 7 \\
How much physical discomfort do you usually experience & 1 & 2 & 3 & 4 & 5 & 6 & 7 \\
\hline
\end{tabular}

When using this specific piece of exercise equipment....

\begin{tabular}{lllllllll}
\hline & \multicolumn{2}{l}{$\begin{array}{l}\text { None at } \\
\text { all }\end{array}$} & & & A lot \\
& & & & \\
\hline How much shoulder pain did you experience & 1 & 2 & 3 & 4 & 5 & 6 & 7 \\
How much bodily pain did you experience & 1 & 2 & 3 & 4 & 5 & 6 & 7 \\
How much physical discomfort did you experience & 1 & 2 & 3 & 4 & 5 & 6 & 7 \\
\hline
\end{tabular}

Opinions and Recommendations

\begin{tabular}{lll|llllr}
\hline & Not at all & & A lot \\
\hline $\begin{array}{l}\text { How much did you like using this specific piece of exercise } \\
\text { equipment? }\end{array}$ & 1 & 2 & 3 & 4 & 5 & 6 & 7 \\
\hline
\end{tabular}

How confident are you that you can use this specific piece of exercise equipment:

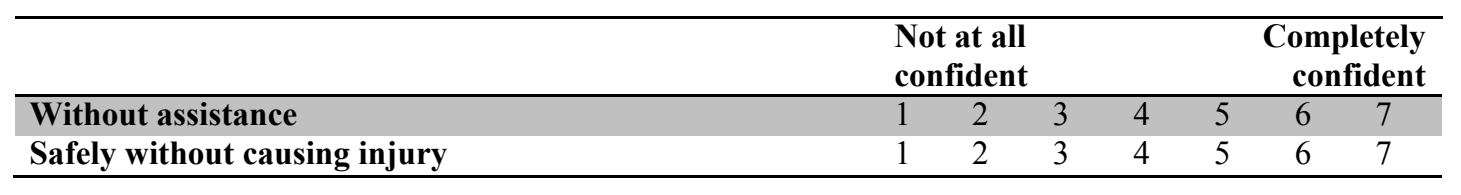

\begin{tabular}{|c|c|c|c|}
\hline & Never & & Always \\
\hline $\begin{array}{l}\text { If the exercise facility you attended had this piece of } \\
\text { equipment, how often would you use it }\end{array}$ & $\begin{array}{lll}1 & 2 & 3\end{array}$ & 45 & 6 \\
\hline
\end{tabular}


PhD Thesis - Chelsea A. Pelletier - McMaster University - Kinesiology

\begin{tabular}{llllllll}
\hline & \multicolumn{1}{c}{$\begin{array}{l}\text { Definitely } \\
\text { not }\end{array}$} & & \multicolumn{3}{c}{$\begin{array}{c}\text { Definitely } \\
\text { yes }\end{array}$} \\
\hline $\begin{array}{l}\text { Would you recommend that exercise facilities purchase } \\
\text { this specific piece of exercise equipment }\end{array}$ & 1 & 2 & 3 & 4 & 5 & 6 & 7 \\
\hline
\end{tabular}

\begin{tabular}{lllllcc}
\hline & Not at all & & \multicolumn{2}{c}{ Very much } \\
\hline $\begin{array}{l}\text { How useful would this piece of equipment be for } \\
\text { improving your fitness to help you perform of your } \\
\text { activities of daily living }\end{array}$ & & & & & & \\
\hline
\end{tabular}

In your opinion, who could safely use this specific piece of exercise equipment (check all that apply)

\begin{tabular}{|l|l|l|}
\hline Exercise status & Injury Characteristics & Other \\
\hline O New exercisers & $\begin{array}{l}\text { O ASIA A O ASIA B O ASIA C } \\
\text { O ASIA D }\end{array}$ & \\
\hline Oxperienced exercises & $\bigcirc$ Paraplegia O Tetraplegia & \\
\hline $\begin{array}{l}\text { O Someone with low fitness } \\
\text { levels }\end{array}$ & & \\
\hline $\begin{array}{l}\text { O Someone with high fitness } \\
\text { levels }\end{array}$ & & \\
\hline
\end{tabular}

Do you have any other comments about this specific piece of exercise equipment to share? 
PhD Thesis - Chelsea A. Pelletier - McMaster University - Kinesiology

$\underline{\text { Aerobic Equipment }}$

In your opinion, what is the most appropriate use for this piece of equipment (check all that apply):
$\bigcirc$ Warm-up
$\bigcirc$ Aerobic training
Cool down
No use whatsoever
$\bigcirc$ Unsure

Assuming that you are very motivated and fit, for how many minutes could you imagine yourself using this specified piece of exercise equipment without stopping?
$\bigcirc$ less than $5 \mathrm{~min}$
$\bigcirc \mathrm{min}$
$10 \mathrm{~min}$
$\bigcirc 15 \mathrm{~min}$
$\bigcirc 20 \mathrm{~min}$
$\bigcirc 25 \mathrm{~min}$
$30 \mathrm{~min}$
Other:

Assuming that you are very motivated and fit, how many times per week could you imagine yourself using this specific piece of exercise equipment?
$\bigcirc 1$
$\bigcirc 2$
$\bigcirc 3$
○ 4
$\bigcirc$ more than 4 
PhD Thesis - Chelsea A. Pelletier - McMaster University - Kinesiology

$\underline{\text { Resistance Equipment }}$

In your opinion, what is the most appropriate use for this piece of equipment (check all that apply):
$\bigcirc$ Warm-up
Increasing muscle strength
$\bigcirc$ Cool down
No use whatsoever
$\bigcirc$ Unsure

Assuming that you are very motivated, in one exercise session, how many sets could you imagine doing while using this specific piece of exercise equipment?
$\bigcirc$ less than 1
$\bigcirc 1$
$\bigcirc 2$
$\bigcirc 3$
$\bigcirc$ more than 3

Assuming that you are very motivated, in one exercise session, how many repetitions could you imagine doing in each set using this specific piece of exercise equipment?
less than 5
5-8
○-10
O $10-12$
O $12-15$
$\bigcirc$ more than 15

Assuming that you are very motivated and fit, how many times per week could you imagine yourself using this specific piece of exercise equipment?
○ 1
○ 2
○ 3
○ 4
$\bigcirc$ more than 4 


\section{Chapter 5}

D.1 Satisfaction with the Guidelines Questionnaire

Considering the exercise program you have been completing over the last 4 months, please rate the following statements:

\begin{tabular}{|c|c|c|c|c|c|c|c|}
\hline & \multicolumn{5}{|c|}{$\begin{array}{l}\text { Strongly } \\
\text { Disagree }\end{array}$} & \multicolumn{2}{|c|}{$\begin{array}{r}\text { Strongly } \\
\text { Agree }\end{array}$} \\
\hline The exercise program was easy to fit into my schedule & 1 & 2 & 3 & 4 & 5 & 6 & 7 \\
\hline The exercise program was appropriate for my ability & 1 & 2 & 3 & 4 & 5 & 6 & 7 \\
\hline $\begin{array}{l}\text { I was able to complete the exercises without any additional pain or } \\
\text { discomfort }\end{array}$ & 1 & 2 & 3 & 4 & 5 & 6 & 7 \\
\hline $\begin{array}{l}\text { I was able to complete each exercise session in a reasonable amount } \\
\text { of time }\end{array}$ & 1 & 2 & 3 & 4 & 5 & 6 & 7 \\
\hline Overall, I enjoyed the exercise program & 1 & 2 & 3 & 4 & 5 & 6 & 7 \\
\hline
\end{tabular}

The next few questions ask about the AEROBIC exercise. This includes the exercise that you did that increased your heart rate and breathing such as arm biking, NuStep, and the vita glide.

\begin{tabular}{|c|c|c|c|c|c|c|c|}
\hline \multirow[b]{2}{*}{ The time of each exercise session was easy for me to complete } & \multicolumn{5}{|c|}{$\begin{array}{l}\text { Strongly } \\
\text { Disagree }\end{array}$} & \multicolumn{2}{|c|}{$\begin{array}{r}\text { Strongly } \\
\text { Agree }\end{array}$} \\
\hline & 1 & 2 & 3 & 4 & 5 & 6 & 7 \\
\hline The intensity was appropriate & 1 & 2 & 3 & 4 & 5 & 6 & 7 \\
\hline The types of exercise I performed were appropriate for my ability & 1 & 2 & 3 & 4 & 5 & 6 & 7 \\
\hline I enjoyed the types of exercise that I completed & 1 & 2 & 3 & 4 & 5 & 6 & 7 \\
\hline The exercise program improved my physical fitness & 1 & 2 & 3 & 4 & 5 & 6 & 7 \\
\hline
\end{tabular}

The next few questions ask about STRENGTHENING activities. These are activities that work your muscles, such as lifting weights and using the pulleys.

\begin{tabular}{|c|c|c|c|c|c|c|c|}
\hline & \multicolumn{5}{|c|}{$\begin{array}{l}\text { Strongly } \\
\text { Disagree }\end{array}$} & \multicolumn{2}{|c|}{$\begin{array}{r}\text { Strongly } \\
\text { Agree }\end{array}$} \\
\hline The number of exercises I did was appropriate & 1 & 2 & 3 & 4 & 5 & 6 & 7 \\
\hline The amount of weight I lifted was appropriate & 1 & 2 & 3 & 4 & 5 & 6 & 7 \\
\hline The types of exercises I performed were appropriate for my ability & 1 & 2 & 3 & 4 & 5 & 6 & 7 \\
\hline The number of repetitions of each exercise was suitable & 1 & 2 & 3 & 4 & 5 & 6 & 7 \\
\hline The exercise program increased my strength & 1 & 2 & 3 & 4 & 5 & 6 & 7 \\
\hline
\end{tabular}

